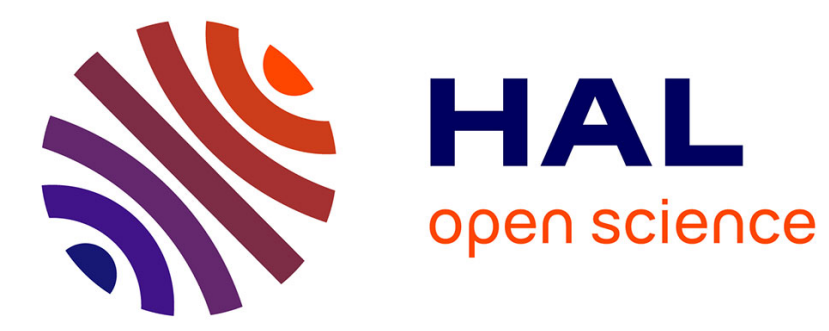

\title{
Atypical lone pair- $\pi$ interaction with quinone methides in a series of imido-ferrociphenol anticancer drug candidates
}

Yong Wang, Pascal Pigeon, Siden Top, Juan Garcia, Claire Troufflard, Ilaria Ciofini, Michael James Mcglinchey, Gérard Jaouen

\section{To cite this version:}

Yong Wang, Pascal Pigeon, Siden Top, Juan Garcia, Claire Troufflard, et al.. Atypical lone pair$\pi$ interaction with quinone methides in a series of imido-ferrociphenol anticancer drug candidates. Angewandte Chemie International Edition, 2019, 58, pp.8421-8425. 10.1002/anie.201902456 . hal02103918

\section{HAL Id: hal-02103918 https://hal.sorbonne-universite.fr/hal-02103918}

Submitted on 19 Apr 2019

HAL is a multi-disciplinary open access archive for the deposit and dissemination of scientific research documents, whether they are published or not. The documents may come from teaching and research institutions in France or abroad, or from public or private research centers.
L'archive ouverte pluridisciplinaire HAL, est destinée au dépôt et à la diffusion de documents scientifiques de niveau recherche, publiés ou non, émanant des établissements d'enseignement et de recherche français ou étrangers, des laboratoires publics ou privés. 


\section{Atypical lone pair- $\pi$ interaction with quinone methides in a series of imido- ferrociphenol anticancer drug candidates}

Yong Wang, ${ }^{1,2}$ Pascal Pigeon, ${ }^{1,2}$ Siden Top, ${ }^{2}$ Juan Sanz García, ${ }^{1}$ Claire Troufflard, ${ }^{2}$ Ilaria Ciofini, ${ }^{1}$ Michael J. McGlinchey, ${ }^{3}$ Gérard Jaouen. ${ }^{* 1,2}$

${ }^{1}$ PSL, Chimie ParisTech, 11 rue Pierre et Marie Curie, F-75005 Paris, France

${ }^{2}$ Sorbonne Université, UPMC Univ Paris 6, UMR 8232 CNRS, IPCM, place Jussieu, F-75005 Paris, France

${ }^{3}$ UCD School of Chemistry, University College Dublin, Belfield, Dublin 4, Ireland

E-mail: gerard.jaouen@upmc.fr, michael.mcglinchey@ucd.ie 


\begin{abstract}
:
Ferrociphenols, especially those possessing a heterocycle at the terminus of an aliphatic chain, display strong anticancer activity via a novel redox mechanism that generates active metabolites such as quinone methides (QMs). X-ray crystallography and UV-Vis spectroscopy reveal that the specific lone pair (lp)- $\pi$ interaction between a carbonyl group of the imide and the quinone motif of the QM plays an important role in the exceptional cytotoxic behaviour of their imido-ferrociphenol precursors. This intramolecular $1 \mathrm{p}-\pi$ interaction markedly enhanced the stability of the QMs and lowered the pKa values of the corresponding phenolates. As the first example of such a non-covalent interaction that stabilizes QMs remotely, it not only expands the scope of the $1 p-\pi$ interaction in supramolecular chemistry, but also represents a new mode of stabilization of a QM. This unprecedented application of $1 \mathrm{p}-\pi$ interactions in imido-ferrociphenol anticancer drug candidates may also have great potential in drug discovery and organocatalyst design.
\end{abstract}

\title{
TOC
}

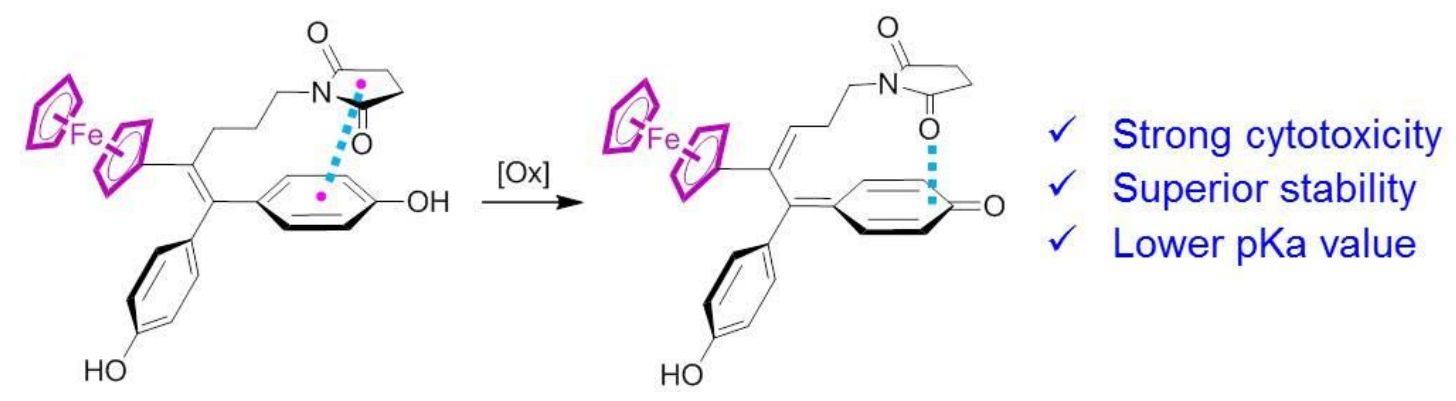


With the goal of circumventing the drawbacks ${ }^{[1]}$ associated with the widely used coordination complexes of $\mathrm{Pt}^{2+}$, the development of metallodrugs based on transition metal bioorganometallic chemistry has gradually come to the fore. ${ }^{[2]}$ Amongst the organometallic complexes that can be used as antitumoral agents, those of iron, an abundant and cheap metal, occupy a privileged position, mostly featuring ferrocene, a compact, stable, non-toxic metallocene showing reversible redox properties. ${ }^{[3]} \mathrm{We}$ have established the concept of a possible use of ferrocenes in oncology based on an entirely new foundation exemplified by the so-called ferrocifen family (Fig. 1a), such as $\mathbf{1}$ and $\mathbf{2}$, whose $\mathrm{IC}_{50}$ values against triple-negative breast cancer (TNBC) cell lines (MDA-MB-231) are 0.6 and $0.5 \mu \mathrm{M}$, respectively. These singular entities possess a redox motif of the [ferrocenyl-ene-phenol] type giving rise to ROS in cancer cells. The initial reversible oxidation of the ferrocenyl antenna permits electronic delocalization leading to selective electrophilic quinone methides (QMs, see below) that induce senescence and/or apoptosis, depending on particular parameters. ${ }^{[3 a]}$
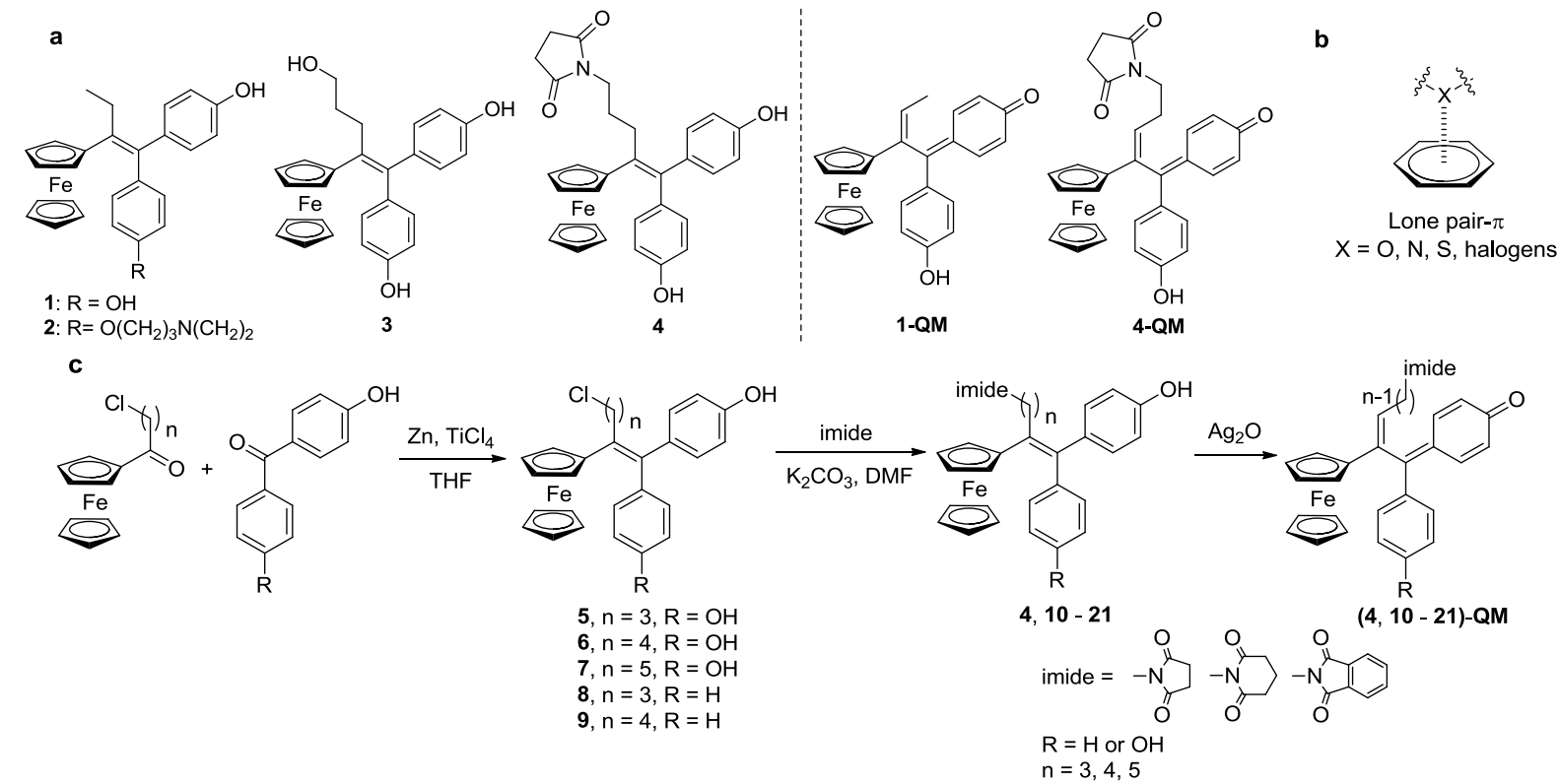

Fig. 1. (a) Ferrocifen derivatives and corresponding QMs. (b) The lone pair- $\pi$ (lp- $\pi$ ) interaction geometry. (c) Syntheses of imido-ferrociphenols and their derived QMs.

We have recently reported the functionalization of the alkyl chain of $\mathbf{1}$ by attaching a terminal hydroxyl substituent ${ }^{[4]}$ or a heterocyclic group, ${ }^{[5]}$ as in $\mathbf{3}$ or $\mathbf{4}$. This latter modification resulted in 
extremely low $\mathrm{IC}_{50}$ values $\left(\mathrm{IC}_{50}=0.035 \mu \mathrm{M}\right)$ on $\mathrm{TNBC}$ MDA-MB-231 cells; in addition, for the epithelial ovarian cancer cell lines A2780 and the cisplatin resistant species A2780cis R the $\mathrm{IC}_{50}$ values were 0.035 and $0.049 \mu \mathrm{M}$, respectively. These data encouraged us to seek a rationale for this exceptional antiproliferative effect. The first metabolite obtained either in the chemical $\left(\mathrm{Ag}^{+}\right) \mathrm{or}^{\mathrm{s}}$ enzymatic oxidation of $\mathbf{1}$ in cancer cells is the quinone methide 1-QM, shown in Fig. 1a, that can react via a 1,8-Michael addition with selective nucleophiles in the cancer cells. ${ }^{[6]}$ The mechanism of action of 4 may follow the similar pathway to that of $\mathbf{1}$.

The lone pair- $\pi$ (lp- $\pi$ ) interaction, depicted schematically in Fig. 1b, refers to the stabilizing association between a lone pair of electrons and the face of a $\pi$ system, and was first invoked in 1995 for the stabilization of Z-DNA. ${ }^{[7]}$ It is now widely recognised as a new supramolecular bond. ${ }^{[8]}$ Although perhaps counterintuitive, and anticipated to be quite weak, its significance has been noted in biological macromolecules $^{[9]}$ and host-guest systems ${ }^{[10]}$. However, relatively few theoretical and experimental studies have elucidated the strength and nature of $1 p-\pi$ interactions, ${ }^{[11]}$ mostly on simplified binding systems of two small molecules, ${ }^{[12]}$ or rigid structures such as triptycene ${ }^{[13]}$ or dibenzobicyclo[3.2.2]nonane, ${ }^{[14]}$ as well as virtual applications in medicinal chemistry or organocatalysis. ${ }^{[15]}$ Gratifyingly, an unprecedented intramolecular $1 \mathrm{p}-\pi$ interaction between an oxygen of imide group and the quinone motif in ferrocenyl QMs is reported herein; this specific interaction affects the stability profiles and the pKa values of QMs, and eventually provides an explanation for the exceptional cytotoxicities of their corresponding ferrociphenol precursors.

The molecules 10 through 21 (see Fig. 1c and Table 2), in which the basic ferrocifen skeleton bears a succinimido, glutarimido or phthalimido substituent at the terminus of a three-, four- or fivecarbon alkyl chain, and the aryl groups are either mono- or di-phenolic, have been prepared in moderate to high yields via a McMurry coupling followed by displacement of chloride by the appropriate imide under basic conditions. The ferroci-diphenols $\mathbf{4 , 1 2}$ and 14, and also the ferroci-monophenols 16,18 and 20, were initially investigated by a combination of ${ }^{1} \mathrm{H}$ and ${ }^{13} \mathrm{C}$ 2D-NMR techniques (Fig. SI1-6). 
Typically, NOESY measurements on the $Z$ isomer of monophenol 16, (identified as such by a correlation between $\mathrm{H}(13 / 17)$ in the phenol ring with the protons in the adjacent cyclopentadienyl ring) in which a succinimido substituent is sited at the end of the propyl chain, revealed a strong correlation between the methylene protons of the heterocycle with the phenyl protons at $\mathrm{C}(8), \mathrm{C}(9)$ and $\mathrm{C}(10)$. Likewise, in the phthalimido complex, $\mathbf{2 0}(Z)$ there is a clear NOE correlation between the protons in the aromatic fragment of the heterocycle and the phenyl protons at $C(8), C(9)$ and $C(10)$.
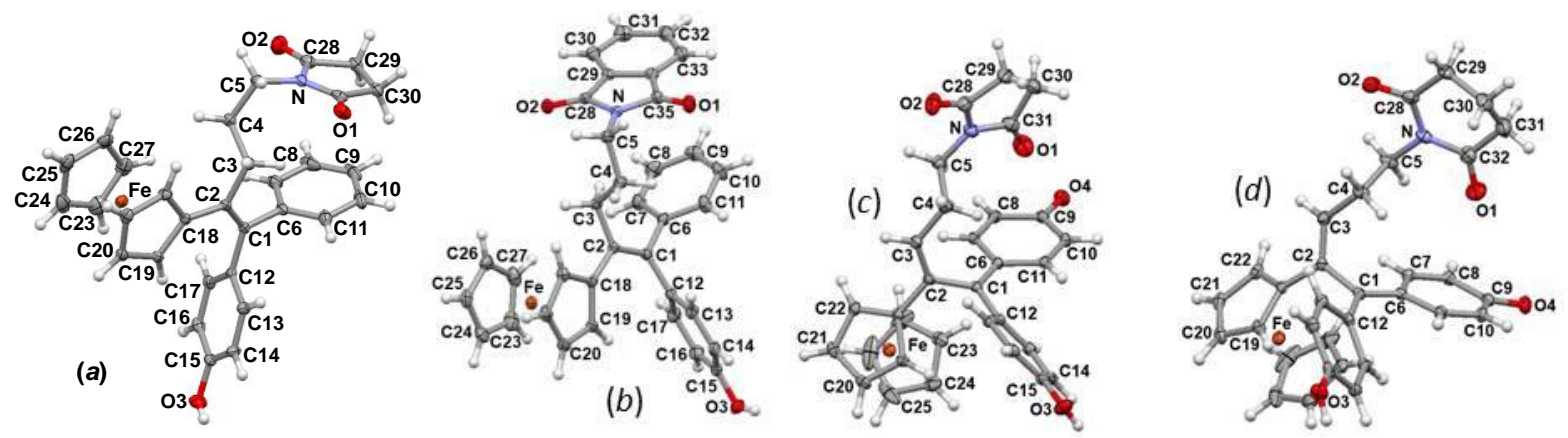

Fig. 2. X-ray crystal structures of ferrociphenols and QMs. $(a)$ 16(Z); $(b) \mathbf{2 0}(Z)$; (c) 4-QM; (d) 12QM.

Gratifyingly, the X-ray crystal structures of $16(Z), \mathbf{2 0}(Z)$ and $\mathbf{1 4}(Z)$ are in excellent accord with the geometries indicated from their NOESY spectra. As shown in Figs. 2a and 2b (see Fig. SI7 for 14), the heterocyclic and phenyl rings in $\mathbf{1 6}(Z)$ deviate from parallelism by only $13.7^{\circ}$, and the vertical distance from the centre of the succinimido ring to the phenyl plane is $3.839 \AA$. Similarly, in $\mathbf{2 0 / ( 1 4 )}$ the dihedral angles between the phthalimido and aryl rings are $16.5^{\circ} /\left(14.8^{\circ}\right)$, and the vertical distances from the centre of the imido ring to the phenyl plane are 3.981/(3.972) $\AA$. Interestingly, in molecules $\mathbf{1 7}$ and 11 whereby the alkyl chain has been lengthened to four and five carbons, respectively, there are no obvious NOE correlations between the methylene protons of the succinimido group with the phenol or phenyl rings (Fig. SI8-9). This observation correlates with the X-ray crystal structure of 17 (Fig. SI7) in which the imido substituent is oriented well away from the other aryl rings.

Treatment of the ferroci-diphenols 4, 10-15, with silver oxide yielded the corresponding monohydroxy QMs, that were again characterized by utilising the full gamut of 2D techniques. 
Surprisingly, at room temperature the NMR data for these QMs were not as well-defined as those derived from the ferroci-monophenols 16-21; however, at $-30{ }^{\circ} \mathrm{C}$ well-resolved spectra were obtained, presumably as the result of the slowing of an exchange process (Fig. SI10-11). To rationalize this observation, which is much more evident in concentrated solutions, one can envisage intermolecular proton exchange between the phenolic proton in one hydroxy-QM and the quinone unit in its counterpart. (details see Fig. SI12).

We note in particular that the propyl chain systems (4-QM, 12-QM, 14-QM) exhibited a markedly greater stability in acetone- $d_{6}$ than did their counterpart QMs with four (10-QM, 13-QM, 15QM) or five (11-QM) carbons in the alkyl chain. Indeed, these latter QMs rearranged to the corresponding ferrocenyl-indenes as the sole product in a few days, or occasionally even in a few hours; they were characterized by comparison of their ${ }^{1} \mathrm{H}$ and ${ }^{13} \mathrm{C}$ NMR spectra with previously reported authentic samples (Fig. SI13-19). ${ }^{[6]}$ In contrast, 4-QM, 12-QM, 14-QM exhibited half-lives in acetone$d_{6}$ of at least a week or more, before finally forming multiple products. Evidently, the stability of these QMs, and their ultimate fate, is exquisitely sensitive to the length of the aliphatic chain.

The NOESY spectra of the propyl-chain QMs differ dramatically from those of their ferrociphenol precursors (Fig. SI20-21). Typically, in complex 16-QM, the correlation between the methylene protons of the succinimido moiety and the adjacent quinone methide ring has been lost; moreover, the methylene group at $\mathrm{C}(4)$ is clearly proximate to the protons sited meta to the ketone group in the QM ring. These observations are readily rationalized upon examination of the X-ray crystal structure of 4-QM (Fig. 2) which revealed that the succinimido ring is now oriented almost perpendicular to the quinone methide. The nearer carbonyl oxygen of the succinimido group is positioned 3.250 $\AA$ from the plane of the QM ring. Likewise, in 12-QM, the glutarimido ring adopts an orientation whereby one of its carbonyl oxygens is now $3.278 \AA$ from the plane of the QM ring. There is evidently a clear change in geometry from the precursor ferrociphenols, exemplified by the X-ray data 
on 14, 16 and 20, in which the structures appear to indicate a $\pi$ stacking interaction between the almost parallel heterocyclic and adjacent aromatic rings, and those observed for 4-QM and 12-QM, in which a carbonyl group is oriented towards the centre of the QM ring. One can most reasonably interpret this result in terms of a lone pair $\bullet \bullet$ arene interaction.

The behaviour of the ferrocifenyl QMs under nearly physiological conditions (50 mM phosphate buffer, $37^{\circ} \mathrm{C}$ ) was conveniently followed by UV-Vis spectroscopy that revealed a distinct colour change from yellow $\left(\lambda_{\max } \cong 430 \mathrm{~nm}\right)$ in the neutral species to bright pink $\left(\lambda_{\max } \cong 560 \mathrm{~nm}\right)$ in the phenolate form, whereby the negative charge is delocalized over both phenoxy rings (Fig. SI22). ${ }^{[16]}$ These findings are confirmed by theoretical calculations performed at TDDFT level (Fig. SI23) displaying a significant red-shift after deprotonation and clearly pointing out the involvement of the phenol/phenolate rings in the intense $\pi-\pi *$ transition (Table SI1). Application of the Beer-Lambert Law in conjunction with the Henderson-Hasselbalch equation yielded $\mathrm{pK}_{\mathrm{a}}$ data (Fig. 3) that clearly showed an increase in the series succinimido $<$ glutarimido $<$ phthalimido. Moreover, these values further increased as the chain was lengthened from propenyl (4-QM, 12-QM, 14-QM) to butenyl (11-QM, 13QM, 15-QM) to pentenyl (10-QM). All these values are smaller than the $\mathrm{pK}_{\mathrm{a}}$ of 9.1 found for $\mathbf{1 - Q M}$ which lacks a heterocyclic terminus. We note in particular, the relative ease of deprotonation of those QMs in which there is a lp- $\pi$ interaction, whereby a carbonyl oxygen is oriented towards the strongly electrophilic quinone methide moiety. These properties are also reflected in their relative stabilities; typically, the half-lives of 4-QM and 12-QM are approximately 40 mins at $\mathrm{pH}$ 5, whereas $\mathbf{1 - Q M}$ and 10-QM are soon transformed into ferrocenyl-indenes ( $\mathrm{t}_{1 / 2}$ is around 10 mins, see Table SI2). One can see clearly that the involvement of lp- $\pi$ interactions improved the stability profiles of QMs under acidic conditions. 


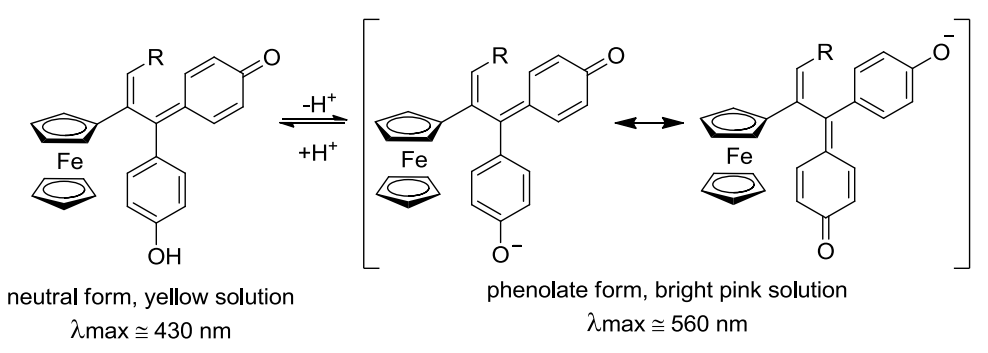

\begin{tabular}{|c|c|c|c|c|c|}
\hline Comp. & $\mathrm{pKa}$ & $\mathrm{IC}_{50}(\mu \mathrm{M})^{\mathrm{a}}$ & Comp. & $\mathrm{pKa}$ & $\mathrm{IC}_{50}(\mu \mathrm{M})^{\mathrm{a}}$ \\
\hline 1-QM & $9.10 \pm 0.15$ & $11 \pm 0.6$ & 12-QM & $8.16 \pm 0.07$ & $0.64 \pm 0.06$ \\
\hline 4-QM & $7.90 \pm 0.12$ & $0.56 \pm 0.2$ & 13-QM & $8.53 \pm 0.10$ & $2.98 \pm 0.26$ \\
\hline 10-QM & $8.30 \pm 0.12$ & $2.12 \pm 0.11$ & 14-QM & $8.63 \pm 0.01$ & $0.77 \pm 0.12$ \\
\hline 11-QM & $8.70 \pm 0.02$ & $3.94 \pm 0.35$ & 15-QM & $8.94 \pm 0.03$ & $7.89 \pm 0.27$ \\
\hline
\end{tabular}

Fig. 3. Acid-base equilibria in the ferrocifenyl-QM systems. The pKa values of QMs $(25 \mu \mathrm{M})$ were measured at $37{ }^{\circ} \mathrm{C}$ in phosphate buffer. The succinimido, glutarimido, and phthalimido QMs are arranged in order of increasing chain length $\left(\mathrm{CH}_{2}\right)_{n}$, where $\mathrm{n}=3,4,5$. (a) Measured after 5 days of culture (mean of two independent experiments \pm SD).

Turning now to the bioactivity of the new imido-ferrociphenols, the results of cytotoxicity tests on MDA-MB-231 cancer cells are listed in Table 2. One can see that $\mathbf{4}$ and $\mathbf{1 6}$ each possessing a threecarbon chain and a succinimido ring showed the most potent cytotoxicity. Replacement of the succinimide ring by a glutarimide or phthalimide ring, as in $\mathbf{1 2}, 18$ or 14,20 , resulted in significant loss of activity. Even more strikingly, incorporation of additional methylene units from 3 to 4 or 5 , as in $\mathbf{4}$, 10 and 11, triggers >10-fold of loss of activity. A similar decrease in cytotoxicity was observed for glutarimido compounds 12, 13 and phthalimido compounds 14, 15. Evidently, the structure activity relationships of ferrociphenol precursors are in excellent accord with the strength of the lp- $\pi$ interaction of their corresponding QMs based on the observed stability profiles and pKa values. We suggest that the most active molecules are those in which the alkyl chain length is such as to position a carbonyl of the heterocycle proximate to the quinone motif of corresponding QMs.

Table 2. $\mathrm{IC}_{50}$ values for selected ferrocenyl compounds towards MDA-MB-231 cells.

\begin{tabular}{|c|c|c|c|c|c|}
\hline Imides & $\begin{array}{l}\text { Chain } \\
\text { length }\end{array}$ & $\begin{array}{l}\text { Ferroci- } \\
\text { diphenols }\end{array}$ & $\mathrm{IC}_{50}(\mu \mathrm{M})^{\mathrm{a}}$ & $\begin{array}{c}\text { Ferroci- } \\
\text { monophenols }\end{array}$ & $\mathrm{IC}_{50}(\mu \mathrm{M})^{\mathrm{a}}$ \\
\hline
\end{tabular}




\begin{tabular}{|c|c|c|c|c|c|}
\hline & & $\mathrm{R}=\mathrm{OH}$ & & $\mathrm{R}=\mathrm{H}$ & \\
\hline & 3 & $4^{b}$ & $0.035 \pm 0.005$ & 16 & $0.035 \pm 0.015$ \\
\hline & 4 & 10 & $0.3 \pm 0.08$ & 17 & $0.47 \pm 0.01$ \\
\hline & 5 & 11 & $0.43 \pm 0.07$ & -- & -- \\
\hline & 3 & 12 & $0.07 \pm 0.01$ & 18 & $0.09 \pm 0.01$ \\
\hline & 4 & 13 & $0.29 \pm 0.01$ & 19 & $0.53 \pm 0.01$ \\
\hline & 3 & $14^{\mathrm{b}}$ & $0.145 \pm 0.005$ & 20 & $0.41 \pm 0.01$ \\
\hline $0^{\prime \prime}$ & 4 & 15 & $0.78 \pm 0.06$ & 21 & $3.59 \pm 0.16$ \\
\hline
\end{tabular}

(a) Measured after 5 days of culture (mean of two independent experiments \pm SD); (b) Values taken from ref. 24.

The antiproliferative activity of selected QMs was also tested on MDA-MB-231 cells. (Table 1)

One might perhaps have anticipated a much weaker effect than was found for the corresponding precursors considering their long-term instability in the assay medium and possible problems of cell penetration. Once again, however, 4-QM and 12-QM exhibited stronger cytotoxicity than all the QMs that we have previously reported ${ }^{[4,6,17]}$ indeed, their cytotoxicity was even comparable to that of some ferrociphenol precursors. This could explain the excellent antitumor activities of $\mathbf{4}$ and $\mathbf{1 2}$ since their QMs may exhibit even more potent antiproliferative effects when formed inside the cell, close to important cell targets.

The antiproliferative effects of the succinimidopropyl-ferrociphenol, $\mathbf{4}$, were further evaluated by the National Cancer Institute on the NCI-60 human tumor cell line panel, which consists of approximately 60 cell lines within nine tumor type subpanels. ${ }^{[18]}$ They were compared to those found for the ethyl-ferrociphenol, 1, tamoxifen (TAM) and cisplatin (CDDP) (Fig. 4 and Table SI3). The imido complex $\mathbf{4}$ shows much stronger cytotoxicity than $\mathbf{1}$ in most types of cancer cells, and its mean $\mathrm{GI}_{50}$ value (around $0.1 \mu \mathrm{M}$ ) is approximately five-fold more potent than that of $\mathbf{1}$ (around $0.5 \mu \mathrm{M}$ ); of course, they are all much more potent than tamoxifen and cisplatin. Moreover, $\mathbf{4}$ shows a high potency towards 
a wide range of cancer cell lines, with a particularly high activity against leukemia and CNS cancer cell lines.

\begin{tabular}{|c|c|c|c|c|c|c|c|c|c|c|c|}
\hline Class & Cell line & 4 & 1 & TAM & CDDP & Class & Cell line & 4 & $\mathbf{1}$ & TAM & CDDP \\
\hline \multirow{5}{*}{ Breast } & $\mathrm{MCF}_{7}$ & & & & & \multirow{6}{*}{ CNS } & SF-268 & & & & \\
\hline & MDA-MB- & & & & & & SF-295 & & & & \\
\hline & $\mathrm{HS}_{578 \mathrm{~T}}$ & & & & & & SF-539 & & & & \\
\hline & BT-549 & & & & & & SNB-19 & & & & \\
\hline & $\mathrm{T}-47 \mathrm{D}$ & & & & & & SNB-75 & & & & \\
\hline \multirow{8}{*}{ Lung } & A549/ATCC & & & & & & $\mathrm{U}_{251}$ & & & & \\
\hline & HOP-62 & & & & & \multirow{6}{*}{ Colon } & COLO 205 & & & & \\
\hline & HOP-92 & & & & & & HCC-2998 & & & & \\
\hline & $\mathrm{NCI}-\mathrm{H} 226$ & & & & & & НCT-116 & & & & \\
\hline & $\mathrm{NCI}-\mathrm{H}_{23}$ & & & & & & HCT-15 & & & & \\
\hline & $\mathrm{NCI}-\mathrm{H}_{322} \mathrm{M}$ & & & & & & KM12 & & & & \\
\hline & $\mathrm{NCI}-\mathrm{H}_{4} 6 \mathrm{o}$ & & & & & & SW-620 & & & & \\
\hline & $\mathrm{NCI}-\mathrm{H}_{522}$ & & & & & \multirow{7}{*}{ Ovarian } & IGROV1 $_{1}$ & & & & \\
\hline \multirow{5}{*}{ Leukemia } & CCRF-CEM & & & & & & OVCAR-3 & & & & \\
\hline & HL-6o(TB) & & & & & & OVCAR-4 & & & & \\
\hline & MOLT-4 & & & & & & OVCAR-5 & & & & \\
\hline & RPMI-8226 & & & & & & OVCAR-8 & & & & \\
\hline & SR & & & & & & SK-OV-3 & & & & \\
\hline \multirow[t]{2}{*}{ Prostate } & $P C-3$ & & & & & & NCI/ADR- & & & & \\
\hline & DU-145 & & & & & \multirow{8}{*}{ Melanoma } & LOX IMVI & & & & \\
\hline \multirow{7}{*}{ Renal } & $786-0$ & & & & & & M14 & & & & \\
\hline & A 498 & & & & & & SK-MEL-2 & & & & \\
\hline & ACHN & & & & & & SK-MEL-28 & & & & \\
\hline & RXF 393 & & & & & & SK-MEL-5 & & & & \\
\hline & $\mathrm{SN}_{12} \mathrm{C}$ & & & & & & UACC-257 & & & & \\
\hline & TK-10 & & & & & & UACC-62 & & & & \\
\hline & UO-31 & & & & & & MDA-MB-435 & & & & \\
\hline
\end{tabular}

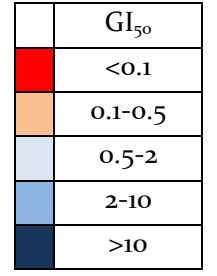

Fig. 4. Heat map for GI $\mathbf{I}_{50}$ values of 4, 1, TAM and CDDP. The cells were treated for $48 \mathrm{~h}$ at five concentrations ranging from 0.01 to $100 \mu \mathrm{M}$. $\mathrm{GI}_{50}, 50 \%$ growth inhibition concentration. The deep red color indicates the highest activity, whereas the deep blue color represents the lowest activity.

To gain more data on the $1 p-\pi$ interactions, whose role appears to be of major significance, a computational study was performed and revealed that the energy differences between conformers that do, or do not, display lp- $\pi$ interactions are extremely small (less than $1 \mathrm{kcal} \mathrm{mol}^{-1}$ in the case of ferrociphenols and corresponding QMs (4, 12, 14, 16, 18 and 20, see Fig. SI24-25). Previous reports on the phenomenon of $1 \mathrm{p}-\pi$ interactions also indicated their strength to be in this range. ${ }^{[7 b, 19]}$ The computed values are at the threshold of accuracy expected for the level of theory used in our calculations, thus allowing us only to conclude that these two conformations have to be considered iso-energetic in 
acetone solution. In the case of QMs containing a $\mathrm{C}_{4}$ chain (that is compounds $10,13,15,17,19$ and 21, see Fig. SI26-27) the parallel $\pi-\pi$ stacked rotamers are found to be more stable, showing once again how delicate is the balance between $\pi$ stacking and the lone pair $\bullet \bullet$ arene interaction in solution and in the solid state. It should also be stressed that the use of empirical dispersion corrections (see SI for computational details) may induce an overestimation of the $\pi-\pi$ stacked conformer's stability.

These computational results were supplemented by variable-temperature (V-T) NMR data to probe the barrier to rotation about the alkyl-chain-succinimido linkage. Thus, 16-QM exists as a racemate in the solid state, but in solution at $203 \mathrm{~K}$, as oscillations within the alkyl chain gradually slow on the NMR time-scale, the emerging chiral character of the enantiomers eventually renders each member of the pairs of methylene protons non-equivalent (Fig. SI28). This diastereotopicity also leads to the development of geminal couplings, and consequent appearance of increasing numbers of peaks. The observed barrier to racemization is $\sim 11.5 \mathrm{kcal} \mathrm{mol}^{-1}$. Most importantly, however, the symmetry of the methylene units in the backbone of the succinimido moiety is never broken in either the ${ }^{1} \mathrm{H}$ or ${ }^{13} \mathrm{C}$ regime; hence, this rotational barrier in solution is very low, in accord with the DFT calculations. (see Table SI4)

In earlier work, we were able to isolate and structurally characterize several ferrocifenyl-QMs by introducing methyl substituents adjacent to the ketonic function. ${ }^{[4,20]}$ In contrast, in 4-QM and 12-QM stabilization is apparently provided only by the $1 \mathrm{p}-\pi$ interaction which is optimized by the length of the alkyl chain and also the rigidity of the system relative to the ferrociphenol precursors. This brings about a dramatic impact not only on the pKa values and stability profile, but more importantly on the bioactivity. To the best of our knowledge, this is the first case of an intramolecular lp- $\pi$ interaction between a carbonyl and a quinone motif; more importantly, it represents the first demonstration of the stabilization of QMs by a remote weak bond interaction. In previous work, this has been accomplished 
either by introducing bulky or electron-donating groups within the quinone scaffold, or by formation of a transition metal quinone complex..$^{[21]}$

One of the mechanisms that we have previously identified for the cytotoxic effect of ferrocenyl phenols is based on their in situ transformation to electrophilic QMs. The greater cytotoxicity of these new ferrociphenols may be explained, at least partly, by the lp- $\pi$ enhanced life-time and stability of their QMs, particularly under weakly acidic conditions. This original temporary stabilizing effect generated on the initial key metabolite is relatively modest from an energetic point of view, but its biological consequences appear to be remarkable since it puts a brake on the rapid chemical dispersion of numerous secondary metabolites that we have identified in a closely related series such as $\mathbf{3}^{25}$ Prolonged drug residence times inside the cell, close to important targets, may result in longer-lasting efficacy, an important factor for drug optimisation. ${ }^{[22]}$ Thus upon oxidative metabolism to QMs, their generation under comparatively mild physiological conditions should allow them to be more aggressively cytotoxic for cancer cells that are known to have a higher level of oxidative stress than normal cells.

The cytotoxicity of a series of imido-ferrociphenols against TNBC cells is shown to correlate strongly with the presence of $1 \mathrm{p}-\pi$ interactions in their corresponding QMs. In cases where this phenomenon is evident from X-ray crystallographic and NMR data, the stability profiles and pKa values suggest that enhancement of the QM life-times is a major factor in determining their antiproliferative activity. This research appears to be the first report of the practical utilization of such a lp- $\pi$ interaction in medicinal chemistry and may have considerable potential in drug discovery. The unprecedented significance of these $1 \mathrm{p}-\pi$ interactions in imido-ferrociphenol anticancer drug candidates merits further exploration, and they are currently under preclinical in vivo evaluation for treatment of intractable cancers.

\section{Acknowledgements}


Y.W. thanks the PGG foundation, PSL University and Feroscan for financial support, and we thank Jérémy Forte and Geoffrey Gontard (IPCM, CNRS UMR 8232) for the X-ray structure determinations, and the National Cancer Institute Developmental Therapeutics Program for in vitro testing. I.C. and J.S.G. received funding from the European Research Council (ERC) under the European Union's Horizon 2020 research and innovation programme (grant agreement No 648558).

Keywords. antitumor agents, non-covalent interactions, bioorganometallics, quinones, ferrocifen.

\section{References}

[1] a) B. Rosenberg, L. Vancamp, Cancer Research 1970, 30, 1799-\&; b) J. J. Wilson, S. J. Lippard, Chem. Rev. 2014, 114, 4470-4495.

[2] a) C. G. Hartinger, P. J. Dyson, Chem. Soc. Rev. 2009, 38, 391-401; b) B. Albada, N. Metzler-Nolte, Chem. Rev. 2016, 116, 11797-11839; c) S. M. Meier-Menches, C. Gerner, W. Berger, C. G. Hartinger, B. K. Keppler, Chem. Soc. Rev. 2018, 47, 909-928; d) P. Zhang, P. J. Sadler, J. Organomet. Chem. 2017, 839, 514.

[3] a) G. Jaouen, A. Vessieres, S. Top, Chem. Soc. Rev. 2015, 44, 8802-8817; b) M. Patra, G. Gasser, Nat. Rev. Chem. 2017, 0066; c) K. Kowalski, Coord. Chem. Rev. 2016, 317, 132-156; d) K. D. Mjos, C. Orvig, Chem. Rev. 2014, 114, 4540-4563.

[4] Y. Wang, P. Pigeon, S. Top, M. J. McGlinchey, G. Jaouen, Angew. Chem. Int. Ed. 2015, 54, 10230-10233.

[5] P. Pigeon, Y. Wang, S. Top, et al, J. Med. Chem. 2017, 60, 8358-8368.

[6] Y. Wang, M-A. Richard, S. Top, et al, Angew. Chem. Int. Ed. 2016, 55, 10431-10434.

[7] a) M. Egli, R. V. Gessner, Proc. Nat. Acad. Sci. 1995, 92, 180; b) M. Egli, S. Sarkhel, Acc. Chem. Res. 2007, 40, 197-205.

[8] a) T. J. Mooibroek, P. Gamez, J. Reedijk, CrystEngComm 2008, 10, 1501-1515; b) S. K. Singh, A. Das, PhysChem ChemPhys 2015, 17, 9596-9612.

[9] a) M. Chawla, E. Chermak, Q. Zhang, et al, Nucleic Acids Research 2017, 45, 11019-11032; b) J. Kozelka, Eur. Biophys. J. 2017, 46, 729-737.

[10] Q.-Q. Wang, N. Luo, X.-D. Wang, et al, J. Am. Chem. Soc. 2017, 139, 635-638.

[11] Z. Badri, C. Foroutan-Nejad, J. Kozelka, R. Marek, PhysChem ChemPhys 2015, 17, 26183-26190.

[12] M.-Z. Ao, Z.-q. Tao, H.-X. Liu, D.-Y. Wu, X. Wang, Comp. Theor. Chem.2015, 1064, 25-34.

[13] B. W. Gung, Y. Zou, Z. Xu, et al, J. Org. Chem. 2008, 73, 689-693.

[14] W. B. Motherwell, R. B. Moreno, I. Pavlakos, et al, Angew. Chem. Int. Ed. 2018, 57, 1193-1198.

[15] A. J. Neel, M. J. Hilton, M. S. Sigman, F. D. Toste, Nature 2017, 543, 637.

[16] V. Scalcon, A. Citta, A. Folda, et al, J. Inorg. Biol. Chem. 2016, 165, 146-151.

[17] Y. Wang, P. M. Dansette, P. Pigeon, et al, Chem. Sci. 2018, 9, 70-78.

[18] R. H. Shoemaker, Nat. Rev. Cancer 2006, 6, 813.

[19] B. W. Gung, X. Xue, H. J. Reich, J. Org. Chem. 2005, 70, 7232-7237.

[20] D. Hamels, P. M. Dansette, E. A. Hillard,et al, Angew. Chem. Int. Ed. 2009, 48, 9124-9126.

[21] S. E. Rokita, Quinone Methides, John Wiley \& Sons, Hoboken, N.J. 2009.

[22] R. A. Copeland, Nat Rev Drug Discov. 2015, 15, 87. 


\section{Supporting Information}

\section{Atypical lone pair- $\pi$ interaction with quinone methides in a series of imido- ferrociphenol anticancer drug candidates}

Yong Wang, Pascal Pigeon, Siden Top, Juan Sanz García, Claire Troufflard, Ilaria Ciofini, Michael J. McGlinchey, * Gérard Jaouen. *

Chemical Synthesis

Computational Details

Biological studies

Figures SI1 - SI28

Tables SI1 - SI5

References 


\section{Chemical Synthesis}

General Synthetic Methods. All reagents and solvents were obtained from commercial suppliers. THF was obtained by distillation from sodium/benzophenone. Thin layer chromatography (TLC) was performed on silica gel $60 \mathrm{GF}_{254}$. Column chromatography was performed on silica gel Merck 60 (40-63 $\mu \mathrm{m}$ ). All NMR experiments $\left({ }^{1} \mathrm{H},{ }^{13} \mathrm{C}, 2 \mathrm{D}\right.$-) were carried out at room temperature on Bruker 300 and 400 NMR spectrometers. Mass spectrometry was performed with a Nermag R 10-10C spectrometer. HRMS measurements were performed on a Thermo Fischer LTQ-Orbitrap XL apparatus equipped with an electrospray source by IPCM (UMR 8232). Elemental analyses were performed by the microanalysis service of ICSN (Gif sur Yvette, France).

General procedure for the synthesis of chloroalkene ferrociphenols. Titanium chloride was added dropwise to a suspension of zinc powder in dry THF at $10-20^{\circ} \mathrm{C}$. The mixture was heated at reflux for 2 hours. A second solution was prepared by dissolving the ferrocenylalkyl compound and the suitable benzophenone in dry THF. This latter solution was added dropwise to the first solution and then the reflux was continued overnight. After cooling to room temperature, the mixture was stirred with water and dichloromethane. The mixture was acidified with dilute hydrochloric acid until the dark color disappeared, and was then decanted. The aqueous layer was extracted with dichloromethane and the combination of organic layers was dried on magnesium sulfate. After concentration under reduced pressure, the crude product was flash-chromatographed on silica gel column with ethyl acetate/cyclohexane $1 / 2$ as the eluent to afford the chloroalkenes ferrociphenols as orange solids that were used in the next steps without further treatment.

7-chloro-2-ferrocenyl-1,1-bis-(4-hydroxyphenyl)-hept-1-en, 7. This compound was synthesized using the general procedure for chloroalkenes ferrociphenols with titanium chloride (1.97 g, $1.14 \mathrm{~mL}, 10.4 \mathrm{mmol})$, zinc powder (1.24 g, $19 \mathrm{mmol})$, dry THF (100 mL), 6-chloro-1-ferrocenylhexan-1-one (1.0 g, $3.14 \mathrm{mmol})$ and 4,4'dihydroxybenzophenone $(0.672 \mathrm{~g}, 3.14 \mathrm{mmol})$ in dry THF $(20 \mathrm{~mL})$. Compound 7 was obtained in a yield of $34 \%$. ${ }^{1} \mathrm{H}$ NMR (300 MHz, acetone- $\left.d_{6}\right): \delta=1.32-1.72\left(\mathrm{~m}, 6 \mathrm{H}, \mathrm{CH}_{2}-\mathrm{CH}_{2}-\mathrm{CH}_{2}\right), 2.57-2.71\left(\mathrm{~m}, 2 \mathrm{H}, \mathrm{CH}_{2}-\mathrm{C}=\mathrm{C}\right), 3.49(\mathrm{t}, J=$ $\left.6.7 \mathrm{~Hz}, 2 \mathrm{H}, \mathrm{CH}_{2} \mathrm{Cl}\right), 3.92\left(\mathrm{t}, J=1.9 \mathrm{~Hz}, 2 \mathrm{H}, \mathrm{C}_{5} \mathrm{H}_{4}\right), 4.06\left(\mathrm{t}, J=1.9 \mathrm{~Hz}, 2 \mathrm{H}, \mathrm{C}_{5} \mathrm{H}_{4}\right), 4.12(\mathrm{~s}, 5 \mathrm{H}, \mathrm{Cp}), 6.70(\mathrm{~d}, J=$ $\left.8.5 \mathrm{~Hz}, 2 \mathrm{H}, \mathrm{C}_{6} \mathrm{H}_{4}\right), 6.81\left(\mathrm{~d}, J=8.5 \mathrm{~Hz}, 2 \mathrm{H}, \mathrm{C}_{6} \mathrm{H}_{4}\right), 6.87\left(\mathrm{~d}, J=8.5 \mathrm{~Hz}, 2 \mathrm{H}, \mathrm{C}_{6} \mathrm{H}_{4}\right), 7.06\left(\mathrm{~d}, J=8.5 \mathrm{~Hz}, 2 \mathrm{H}, \mathrm{C}_{6} \mathrm{H}_{4}\right)$, $8.21(\mathrm{~s}, 1 \mathrm{H}, \mathrm{OH}), 8.25(\mathrm{~s}, 1 \mathrm{H}, \mathrm{OH}) .{ }^{13} \mathrm{C} \mathrm{NMR}\left(75 \mathrm{MHz}\right.$, acetone- $\left.d_{6}\right): \delta=27.5\left(\mathrm{CH}_{2}\right), 30.6\left(\mathrm{CH}_{2}\right), 33.0\left(\mathrm{CH}_{2}\right), 35.2$ $\left(\mathrm{CH}_{2}\right), 45.6\left(\mathrm{CH}_{2}\right), 68.6\left(2 \mathrm{xCH}, \mathrm{C}_{5} \mathrm{H}_{4}\right), 69.8(5 \mathrm{xCH}, \mathrm{Cp}), 70.0\left(2 \mathrm{xCH}, \mathrm{C}_{5} \mathrm{H}_{4}\right), 88.5\left(\mathrm{C}, \mathrm{C}_{5} \mathrm{H}_{4}\right), 115.7(2 \mathrm{xCH}$, $\left.\mathrm{C}_{6} \mathrm{H}_{4}\right), 115.8$ (2xCH, $\left.\mathrm{C}_{6} \mathrm{H}_{4}\right), 131.3\left(2 \mathrm{xCH}, \mathrm{C}_{6} \mathrm{H}_{4}\right), 131.8$ (2xCH, $\left.\mathrm{C}_{6} \mathrm{H}_{4}\right), 135.1(\mathrm{C}), 137.1(\mathrm{C}), 137.4(\mathrm{C}), 139.2$ (C), 156.6 (C), 156.7 (C).

5-chloro-2-ferrocenyl-1-(4-hydroxyphenyl)-1-phenyl-pent-1-ene, 8. This compound was synthesized using the general procedure for chloroalkenes ferrociphenols with titanium chloride (6.73 g, $3.9 \mathrm{~mL}, 35.5 \mathrm{mmol})$, zinc 
powder (4.15 g, $63.5 \mathrm{mmol})$, dry THF (250 mL), 4-chloro-1-ferrocenyl-1-butanone (3.09 g, $10.6 \mathrm{mmol})$ and 4hydroxybenzophenone $(2.11 \mathrm{~g}, 10.6 \mathrm{mmol})$ in dry THF $(40 \mathrm{~mL})$. Compound 8 was obtained in a yield of $47 \% .{ }^{1} \mathrm{H}$ NMR (300 MHz, acetone- $\left.d_{6}\right): \delta=1.86-2.02\left(\mathrm{~m}, 2 \mathrm{H}, \mathrm{CH}_{2}\right), 2.72-2.88\left(\mathrm{~m}, 2 \mathrm{H}, \mathrm{CH}_{2}\right), 3.47$ and $3.51(\mathrm{t}, J=6.6 \mathrm{~Hz}$, $\left.2 \mathrm{H}, \mathrm{CH}_{2}\right), 3.92$ and $3.99\left(\mathrm{t}, J=1.9 \mathrm{~Hz}, 2 \mathrm{H}, \mathrm{C}_{5} \mathrm{H}_{4}\right), 4.07$ and $4.10\left(\mathrm{t}, J=1.9 \mathrm{~Hz}, 2 \mathrm{H}, \mathrm{C}_{5} \mathrm{H}_{4}\right), 4.14$ and $4.15(\mathrm{~s}, 5 \mathrm{H}$, Cp), 6.74 and $6.84\left(\mathrm{~d}, J=8.5 \mathrm{~Hz}, 2 \mathrm{H}, \mathrm{C}_{6} \mathrm{H}_{4}\right), 6.91$ and $7.07\left(\mathrm{~d}, J=8.5 \mathrm{~Hz}, 2 \mathrm{H}, \mathrm{C}_{6} \mathrm{H}_{4}\right), 7.08-7.39(\mathrm{~m}, 5 \mathrm{H}, \mathrm{Ph}), 8.25$ and $8.29(\mathrm{~s}, 1 \mathrm{H}, \mathrm{OH}) .{ }^{13} \mathrm{C} \mathrm{NMR}\left(75 \mathrm{MHz}\right.$, acetone- $\left.d_{6}\right): \delta=32.9$ and $33.0\left(\mathrm{CH}_{2}\right), 35.2$ and $35.3\left(\mathrm{CH}_{2}\right), 45.8$ and $45.9\left(\mathrm{CH}_{2} \mathrm{Cl}\right), 68.9\left(2 \mathrm{xCH}, \mathrm{C}_{5} \mathrm{H}_{4}\right), 69.9\left(2 \mathrm{xCH}, \mathrm{C}_{5} \mathrm{H}_{4}+5 \mathrm{xCH}, \mathrm{Cp}\right), 87.7$ and $87.8\left(\mathrm{C}_{1} \mathrm{C}_{5} \mathrm{H}_{4}\right), 115.9$ and 116.0 $\left(2 \mathrm{xCH}, \mathrm{C}_{6} \mathrm{H}_{4}\right), 126.9$ and $127.0(\mathrm{CH}, \mathrm{Ph}), 128.9$ and $129.1\left(2 \mathrm{xCH}_{\text {arom }}\right), 130.0$ and $130.5\left(2 \mathrm{xCH}_{\text {arom }}\right), 131.2$ and $131.6\left(2 \mathrm{xCH}_{\text {arom }}\right), 134.6$ and $134.9(\mathrm{C}), 136.3$ and $136.7(\mathrm{C}), 139.8$ and $139.9(\mathrm{C}), 145.6$ and $146.0(\mathrm{C}), 156.7$ and $156.8(\mathrm{C})$.

6-chloro-2-ferrocenyl-1-(4-hydroxyphenyl)-1-phenylhex-1-en, 9. This compound was synthesized using the general procedure for chloroalkenes ferrociphenols with titanium chloride (36.804 g, $21.32 \mathrm{~mL}, 194 \mathrm{mmol})$, zinc powder (15.218 g, $232.8 \mathrm{mmol})$, dry THF (600 mL), 5-chloro-1-ferrocenyl-1-pentanone (11.82 g, $38.8 \mathrm{mmol})$ and 4-hydroxybenzophenone (7.691 g, $38.8 \mathrm{mmol})$ in dry THF (100 mL). Compound 9 was obtained in a yield of 59\%. ${ }^{1} \mathrm{H}$ NMR (300 MHz, acetone- $\left.d_{6}\right): \delta=1.57-1.74\left(\mathrm{~m}, 4 \mathrm{H}, \mathrm{CH}_{2}-\mathrm{CH}_{2}\right), 2.58$ and $2.65\left(\mathrm{t}, J=7.4 \mathrm{~Hz}, 2 \mathrm{H}, \mathrm{CH}_{2^{-}}\right.$ $\mathrm{C}=\mathrm{C}), 3.44$ and $3.48\left(\mathrm{t}, J=6.3 \mathrm{~Hz}, 2 \mathrm{H}, \mathrm{CH}_{2} \mathrm{Cl}\right), 3.93$ and $4.01\left(\mathrm{~s}\right.$ broad, $\left.2 \mathrm{H}, \mathrm{C}_{5} \mathrm{H}_{4}\right), 4.09$ and $4.13(\mathrm{~s}$ broad, $2 \mathrm{H}$, $\left.\mathrm{C}_{5} \mathrm{H}_{4}\right), 4.17$ and $4.18(\mathrm{~s}, 5 \mathrm{H}, \mathrm{Cp}), 6.74$ and $6.83\left(\mathrm{~d}, J=8.5 \mathrm{~Hz}, 2 \mathrm{H}, \mathrm{C}_{6} \mathrm{H}_{4}\right), 6.91$ and $7.07(\mathrm{~d}, J=8.5 \mathrm{~Hz}, 2 \mathrm{H}$, $\left.\mathrm{C}_{6} \mathrm{H}_{4}\right), 7.13-7.38(\mathrm{~m}, 5 \mathrm{H}, \mathrm{Ph}) .{ }^{13} \mathrm{C}$ NMR $\left(75 \mathrm{MHz}\right.$, acetone- $\left.d_{6}\right): \delta=28.6$ and $28.7\left(\mathrm{CH}_{2}\right), 33.36$ and $33.39\left(\mathrm{CH}_{2}\right)$, 34.48 and $34.53\left(\mathrm{CH}_{2}\right), 45.5$ and $45.6\left(\mathrm{CH}_{2} \mathrm{Cl}\right), 69.03$ and $69.07\left(2 \mathrm{xCH}, \mathrm{C}_{5} \mathrm{H}_{4}\right), 70.1$ and $70.2\left(2 \times \mathrm{CH}_{2} \mathrm{C}_{5} \mathrm{H}_{4}+\right.$ 5xCH, Cp), $88.2\left(\mathrm{C}, \mathrm{C}_{5} \mathrm{H}_{4}\right), 115.88$ and $115.92\left(2 \mathrm{xCH}, \mathrm{C}_{6} \mathrm{H}_{4}\right), 126.9(\mathrm{CH}, \mathrm{Ph}), 128.9$ and $129.0\left(2 \mathrm{xCH}_{\text {arom }}\right), 130.1$ and $130.6\left(2 \mathrm{xCH}_{\text {arom }}\right), 131.3$ and $131.7\left(2 \mathrm{xCH}_{\text {arom }}\right), 135.5$ and $135.8(\mathrm{C}), 136.5$ and $136.8(\mathrm{C}), 139.6(\mathrm{C}), 145.8$ and $146.2(\mathrm{C}), 156.7$ and 156.8 (C). HRMS (ESI, $\mathrm{C}_{28} \mathrm{H}_{27} \mathrm{ClFeO}$ : [M] $]^{+}$) calcd: 470.1094, found: 470.1091.

General procedure for the synthesis of imido-ferrociphenols. A mixture of potassium carbonate, imide and chloroalkene ferrociphenol in dimethylformamide (DMF) was heated under stirring at $80{ }^{\circ} \mathrm{C}$ overnight. The mixture was allowed to cool to room temperature, poured into a dilute hydrochloric acid solution, and extracted twice with diethyl ether, then the organic layer was dried on magnesium sulfate and concentrated under reduced pressure. The residue was purified by flash-chromatography on silica gel column with a gradient of ethyl acetate/cyclohexane (1/2 to $2 / 1)$ as the eluent and crystallized from the suitable solvent to afford the imides as orange solids.

N-\{6-ferrocenyl-7,7-bis-(4-hydroxyphenyl)-hept-6-enyl\}succinimide (11). This compound was synthesized using the general procedure for imides starting from chloroalkene ferrociphenol 7 (0.349 $\mathrm{g}, 0.697 \mathrm{mmol})$, succinimide $(0.138 \mathrm{~g}, 1.39 \mathrm{mmol})$, potassium carbonate $(0.193 \mathrm{~g}, 1.39 \mathrm{mmol})$, DMF (15 mL) to afford 11 in a yield of $31 \%$. ${ }^{1} \mathrm{H}$ NMR (300 MHz, acetone- $\left.d_{6}\right): \delta=1.15-1.59\left(\mathrm{~m}, 6 \mathrm{H}, \mathrm{CH}_{2}-\mathrm{CH}_{2}-\mathrm{CH}_{2}\right), 2.56-2.70\left(\mathrm{~m}, 6 \mathrm{H}, 2 \mathrm{CH}_{2}\right.$ 
succ $\left.+\mathrm{CH}_{2}-\mathrm{C}=\mathrm{C}\right), 3.36\left(\mathrm{t}, J=7.2 \mathrm{~Hz}, 2 \mathrm{H}, \mathrm{CH}_{2} \mathrm{~N}\right), 3.92\left(\mathrm{t}, J=1.9 \mathrm{~Hz}, 2 \mathrm{H}, \mathrm{C}_{5} \mathrm{H}_{4}\right), 4.05\left(\mathrm{t}, J=1.9 \mathrm{~Hz}, 2 \mathrm{H}, \mathrm{C}_{5} \mathrm{H}_{4}\right)$, $4.11(\mathrm{~s}, 5 \mathrm{H}, \mathrm{Cp}), 6.70\left(\mathrm{~d}, J=8.5 \mathrm{~Hz}, 2 \mathrm{H}, \mathrm{C}_{6} \mathrm{H}_{4}\right), 6.80\left(\mathrm{~d}, J=8.5 \mathrm{~Hz}, 2 \mathrm{H}, \mathrm{C}_{6} \mathrm{H}_{4}\right), 6.87\left(\mathrm{~d}, J=8.5 \mathrm{~Hz}, 2 \mathrm{H}, \mathrm{C}_{6} \mathrm{H}_{4}\right)$, $7.05\left(\mathrm{~d}, J=8.5 \mathrm{~Hz}, 2 \mathrm{H}, \mathrm{C}_{6} \mathrm{H}_{4}\right), 8.17$ (s broad, $\left.1 \mathrm{H}, \mathrm{OH}\right), 8.20$ (s broad, $\left.1 \mathrm{H}, \mathrm{OH}\right) .{ }^{13} \mathrm{C}$ NMR $\left(75 \mathrm{MHz}\right.$, acetone- $\left.d_{6}\right)$ : $\delta=27.6\left(\mathrm{CH}_{2}\right), 28.1\left(\mathrm{CH}_{2}\right), 28.7\left(2 \mathrm{xCH}_{2}\right.$, succinimide), $31.1\left(\mathrm{CH}_{2}\right), 35.3\left(\mathrm{CH}_{2}\right), 38.9\left(\mathrm{CH}_{2}\right), 68.6\left(2 \mathrm{xCH}, \mathrm{C}_{5} \mathrm{H}_{4}\right)$, 69.8 (5xCH, Cp), $70.0\left(2 x \mathrm{CH}, \mathrm{C}_{5} \mathrm{H}_{4}\right), 88.4\left(\mathrm{C}, \mathrm{C}_{5} \mathrm{H}_{4}\right), 115.7\left(2 \mathrm{xCH}, \mathrm{C}_{6} \mathrm{H}_{4}\right), 115.8\left(2 \mathrm{xCH}, \mathrm{C}_{6} \mathrm{H}_{4}\right), 131.3(2 \mathrm{xCH}$, $\left.\mathrm{C}_{6} \mathrm{H}_{4}\right), 131.7$ (2xCH, $\left.\mathrm{C}_{6} \mathrm{H}_{4}\right), 135.2(\mathrm{C}), 137.2(\mathrm{C}), 137.5(\mathrm{C}), 139.1$ (C), $156.5(\mathrm{C}), 156.6$ (C), 178.0 (2xC, C=O). IR (ATR, $\left.v \mathrm{~cm}^{-1}\right)$ : $1686(\mathrm{CO})$. HRMS (ESI, $\mathrm{C}_{33} \mathrm{H}_{33} \mathrm{FeNNaO}_{4}$ : [M+Na] $]^{+}$) calcd: 586.165118, found: 586.1652. Anal. Calcd for $\mathrm{C}_{33} \mathrm{H}_{33} \mathrm{FeNO}_{4}\left(\mathrm{H}_{2} \mathrm{O}\right)$ : C, 68.16; H, 6.06; N, 2.4. Found: C, 68.17; H, 6.31; N, 2.37 .

\section{N-\{5-ferrocenyl-6,6-bis-(4-hydroxyphenyl)-hex-5-enyl\}glutarimide (13). This compound was synthesized} using the general procedure for imides starting from chloroalkene ferrociphenol 6 (1.0 g, $2.05 \mathrm{mmol})$, glutarimide (0.465 g, $4.1 \mathrm{mmol})$, potassium carbonate $(0.568 \mathrm{~g}, 4.1 \mathrm{mmol})$, DMF $(60 \mathrm{~mL})$ to afford 13 in a yield of $7 \%$. ${ }^{1} \mathrm{H}$ NMR (300 MHz, acetone- $\left.d_{6}\right): \delta=1.35-1.49\left(\mathrm{~m}, 4 \mathrm{H}, \mathrm{CH}_{2}-\mathrm{CH}_{2}\right), 1.84-1.95\left(\mathrm{~m}, 2 \mathrm{H}, \mathrm{CH}_{2}\right), 2.56-2.67\left(\mathrm{~m}, 6 \mathrm{H}, 2 \mathrm{CH}_{2}\right.$ glutarimide $\left.+\mathrm{CH}_{2}-\mathrm{C}=\mathrm{C}\right), 3.60\left(\mathrm{t}, J=6.8 \mathrm{~Hz}, 2 \mathrm{H}, \mathrm{CH}_{2} \mathrm{~N}\right), 3.91\left(\mathrm{t}, J=1.9 \mathrm{~Hz}, 2 \mathrm{H}, \mathrm{C}_{5} \mathrm{H}_{4}\right), 4.06(\mathrm{t}, J=1.9 \mathrm{~Hz}, 2 \mathrm{H}$, $\left.\mathrm{C}_{5} \mathrm{H}_{4}\right), 4.13(\mathrm{~s}, 5 \mathrm{H}, \mathrm{Cp}), 6.70\left(\mathrm{~d}, J=8.5 \mathrm{~Hz}, 2 \mathrm{H}, \mathrm{C}_{6} \mathrm{H}_{4}\right), 6.80\left(\mathrm{~d}, J=8.5 \mathrm{~Hz}, 2 \mathrm{H}, \mathrm{C}_{6} \mathrm{H}_{4}\right), 6.86(\mathrm{~d}, J=8.5 \mathrm{~Hz}, 2 \mathrm{H}$, $\left.\mathrm{C}_{6} \mathrm{H}_{4}\right), 7.04\left(\mathrm{~d}, J=8.5 \mathrm{~Hz}, 2 \mathrm{H}, \mathrm{C}_{6} \mathrm{H}_{4}\right), 8.20(\mathrm{~s}, 1 \mathrm{H}, \mathrm{OH}), 8.23(\mathrm{~s}, 1 \mathrm{H}, \mathrm{OH}) .{ }^{13} \mathrm{C}$ NMR $\left(75 \mathrm{MHz}, \mathrm{DMSO}-d_{6}\right): \delta=$ $18.0\left(\mathrm{CH}_{2}\right.$, glutarimide), $28.8\left(\mathrm{CH}_{2}\right), 29.0\left(\mathrm{CH}_{2}\right), 33.3\left(2 \mathrm{xCH}_{2}\right.$, glutarimide), $35.3\left(\mathrm{CH}_{2}\right), 39.5\left(\mathrm{CH}_{2}\right), 68.8(2 \times C H$, $\left.\mathrm{C}_{5} \mathrm{H}_{4}\right), 70.1\left(5 \mathrm{xCH}, \mathrm{Cp}+2 \mathrm{xCH}, \mathrm{C}_{5} \mathrm{H}_{4}\right), 88.5\left(\mathrm{C}, \mathrm{C}_{5} \mathrm{H}_{4}\right), 115.8\left(2 \mathrm{xCH}, \mathrm{C}_{6} \mathrm{H}_{4}\right), 115.9\left(2 x \mathrm{xH}, \mathrm{C}_{6} \mathrm{H}_{4}\right), 131.3(2 x C H$, $\left.\mathrm{C}_{6} \mathrm{H}_{4}\right), 131.8\left(2 \times C H, \mathrm{C}_{6} \mathrm{H}_{4}\right), 135.4(\mathrm{C}), 137.2(\mathrm{C}), 137.6(\mathrm{C}), 139.2(\mathrm{C}), 156.6(\mathrm{C}), 156.7(\mathrm{C}), 172.0(2 \times C, \mathrm{C}=\mathrm{O})$. IR $\left(\mathrm{KBr}, v \mathrm{~cm}^{-1}\right): 1649(\mathrm{CO})$. HRMS (ESI, $\mathrm{C}_{33} \mathrm{H}_{33} \mathrm{FeNO}_{4}$ : [M] $]^{+}$) calcd: 563.1754, found: 563.1752. Anal. Calcd for $\mathrm{C}_{33} \mathrm{H}_{33} \mathrm{FeNO}_{4}\left(\mathrm{H}_{2} \mathrm{O}\right)_{0.1}$ : C, 70.12; H, 5.92; N, 2.47. Found: C, 69.83; H, 6.31; N, 2.19.

N-\{5-ferrocenyl-6,6-bis-(4-hydroxyphenyl)-hex-5-enyl\}phthalimide (15). This compound was synthesized using the general procedure for imides starting from chloroalkene ferrociphenol 6 (3.693 g, $7.59 \mathrm{mmol}$ ), phthalimide (2.232 g, $15.2 \mathrm{mmol})$, potassium carbonate (2.097 g, $15.2 \mathrm{mmol})$, DMF (100 mL) to afford 15 in a yield of $94 \%$. Mp : $101{ }^{\circ} \mathrm{C} .{ }^{1} \mathrm{H}$ NMR $\left(300 \mathrm{MHz}\right.$, acetone- $\left.d_{6}\right): \delta=1.50-1.73\left(\mathrm{~m}, 4 \mathrm{H}, \mathrm{CH}_{2}-\mathrm{CH}_{2}\right), 2.72(\mathrm{t}, J=7.6$ $\left.\mathrm{Hz}, 2 \mathrm{H}, \mathrm{CH}_{2}-\mathrm{C}=\mathrm{C}\right), 3.60\left(\mathrm{t}, J=6.9 \mathrm{~Hz}, 2 \mathrm{H}, \mathrm{CH}_{2} \mathrm{~N}\right), 3.94\left(\mathrm{t}, J=1.9 \mathrm{~Hz}, 2 \mathrm{H}, \mathrm{C}_{5} \mathrm{H}_{4}\right), 4.07\left(\mathrm{t}, J=1.9 \mathrm{~Hz}, 2 \mathrm{H}, \mathrm{C}_{5} \mathrm{H}_{4}\right)$, 4.15 (s, 5H, Cp), $6.72\left(\mathrm{~d}, J=8.5 \mathrm{~Hz}, 2 \mathrm{H}, \mathrm{C}_{6} \mathrm{H}_{4}\right), 6.73\left(\mathrm{~d}, J=8.5 \mathrm{~Hz}, 2 \mathrm{H}, \mathrm{C}_{6} \mathrm{H}_{4}\right), 6.84\left(\mathrm{~d}, J=8.5 \mathrm{~Hz}, 2 \mathrm{H}, \mathrm{C}_{6} \mathrm{H}_{4}\right)$, $6.98\left(\mathrm{~d}, J=8.5 \mathrm{~Hz}, 2 \mathrm{H}, \mathrm{C}_{6} \mathrm{H}_{4}\right), 7.88$ (s, 4H, phthalimide), 8.27 (s, 1H, OH), $8.28(\mathrm{~s}, 1 \mathrm{H}, \mathrm{OH}) .{ }^{13} \mathrm{C} \mathrm{NMR}(75 \mathrm{MHz}$, acetone- $\left.d_{6}\right): \delta=28.2\left(\mathrm{CH}_{2}\right), 28.9\left(\mathrm{CH}_{2}\right), 34.6\left(\mathrm{CH}_{2}\right), 38.0\left(\mathrm{CH}_{2}\right), 68.3\left(2 \mathrm{xCH}, \mathrm{C}_{5} \mathrm{H}_{4}\right), 69.5(5 \mathrm{xCH}, \mathrm{Cp}), 69.7$ $\left(2 \mathrm{xCH}, \mathrm{C}_{5} \mathrm{H}_{4}\right), 88.2\left(\mathrm{C}, \mathrm{C}_{5} \mathrm{H}_{4}\right), 115.4\left(2 \mathrm{xCH}, \mathrm{C}_{6} \mathrm{H}_{4}\right), 115.5\left(2 \mathrm{xCH}, \mathrm{C}_{6} \mathrm{H}_{4}\right), 123.3$ (2xCH, phthalimide), 130.9 $\left(2 \mathrm{xCH}, \mathrm{C}_{6} \mathrm{H}_{4}\right), 131.4\left(2 \mathrm{xCH}, \mathrm{C}_{6} \mathrm{H}_{4}\right), 132.8(2 \mathrm{xC}), 134.6$ (2xCH, phthalimide + C), $136.7(\mathrm{C}), 137.1(\mathrm{C}), 139.1$ (C), 156.3 (C), 156.4 (C), 168.4 (2xC, C=O). IR (KBr, $\left.v \mathrm{~cm}^{-1}\right): 1700$ (CO). MS (EI, $\left.70 \mathrm{eV}\right) \mathrm{m} / \mathrm{z}: 597$ [M] $]^{+}, 532$ [M-Cp] $^{+}$, 382, 343. HRMS (ESI, $\mathrm{C}_{36} \mathrm{H}_{31} \mathrm{FeNO}_{4}$ : [M] $]^{+}$) calcd: 597.1602, found: 597.1617. Anal. Calcd for $\mathrm{C}_{36} \mathrm{H}_{31} \mathrm{FeNO}_{4}\left(\mathrm{H}_{2} \mathrm{O}\right)_{0.5}: \mathrm{C}, 71.29 ; \mathrm{H}, 5.31 ; \mathrm{N}, 2.31$. Found: C, 70.9; H, 5.32; N, 2.26. 
N-\{4-ferrocenyl-5-(4-hydroxyphenyl)-5-phenyl-pent-4-enyl\}succinimide (16). This compound was synthesized using the general procedure for imides starting from chloroalkene ferrociphenol $\mathbf{8}(0.654 \mathrm{~g}, 1.43$ mmol), succimide $(0.426 \mathrm{~g}, 4.3 \mathrm{mmol})$, potassium carbonate $(0.594 \mathrm{~g}, 4.3 \mathrm{mmol})$, DMF (20 mL) to afford 16 in a yield of $84 \% .{ }^{1} \mathrm{H}$ NMR $\left(300 \mathrm{MHz}\right.$, acetone- $\left.d_{6}\right): \delta=1.67-1.81\left(\mathrm{~m}, 2 \mathrm{H}, \mathrm{CH}_{2}\right), 2.50-2.63\left(\mathrm{~m}, 6 \mathrm{H}, 2 \mathrm{CH}_{2}\right.$ succ $+\mathrm{CH}_{2}-$ $\mathrm{C}=\mathrm{C}), 3.33$ and $3.36\left(\mathrm{t}, J=6.6 \mathrm{~Hz}, 2 \mathrm{H}, \mathrm{CH}_{2} \mathrm{~N}\right), 3.86$ and $3.93\left(\mathrm{t}, J=1.9 \mathrm{~Hz}, 2 \mathrm{H}, \mathrm{C}_{5} \mathrm{H}_{4}\right), 4.05$ and $4.09(\mathrm{t}, J=1.9$ $\left.\mathrm{Hz}, 2 \mathrm{H}, \mathrm{C}_{5} \mathrm{H}_{4}\right), 4.13$ and $4.14(\mathrm{~s}, 5 \mathrm{H}, \mathrm{Cp}), 6.72$ and $6.82\left(\mathrm{~d}, J=8.5 \mathrm{~Hz}, 2 \mathrm{H}, \mathrm{C}_{6} \mathrm{H}_{4}\right), 6.90$ and $7.03(\mathrm{~d}, J=8.5 \mathrm{~Hz}$, $\left.2 \mathrm{H}, \mathrm{C}_{6} \mathrm{H}_{4}\right), 7.05-7.40(\mathrm{~m}, 5 \mathrm{H}, \mathrm{Ph}), 8.26$ and $8.35(\mathrm{~s}, 1 \mathrm{H}, \mathrm{OH}) .{ }^{13} \mathrm{C}$ NMR $\left(75 \mathrm{MHz}\right.$, acetone- $\left.d_{6}\right): \delta=28.6$ and 28.7 (2xCH 2 , succ), $29.9\left(\mathrm{CH}_{2}\right), 32.9$ and $33.0\left(\mathrm{CH}_{2}\right), 38.9$ and $39.0\left(\mathrm{CH}_{2}\right), 68.7$ and $68.9\left(2 \mathrm{xCH}, \mathrm{C}_{5} \mathrm{H}_{4}\right), 69.9$ and 70.1 $\left(5 x C H, C p+2 x C H, C_{5} \mathrm{H}_{4}\right), 87.5$ and $87.6\left(C, \mathrm{C}_{5} \mathrm{H}_{4}\right), 115.9$ and $116.0\left(2 x C H, \mathrm{C}_{6} \mathrm{H}_{4}\right), 126.9(\mathrm{CH}, \mathrm{Ph}), 129.0$ and $129.1\left(2 \mathrm{xCH}_{\text {arom }}\right), 130.0$ and $130.5\left(2 \mathrm{xCH}_{\text {arom }}\right), 131.2$ and $131.6\left(2 \mathrm{xCH}_{\text {arom }}\right), 135.1$ and $136.6(\mathrm{C}), 135.6$ and 136.7 (C), 139.5 (C), 145.8 and $146.0(\mathrm{C}), 156.7$ and $156.9(\mathrm{C}), 177.8$ and $177.9(2 \mathrm{xC}, \mathrm{C}=\mathrm{O})$. IR (ATR, $\left.v \mathrm{~cm}^{-1}\right): 1689$ (CO). HRMS (ESI, $\mathrm{C}_{31} \mathrm{H}_{29} \mathrm{FeNNaO}_{3}:[\mathrm{M}+\mathrm{Na}]^{+}$) calcd: 542.138903, found: 542.1389. Anal. Calcd for $\mathrm{C}_{31} \mathrm{H}_{29} \mathrm{FeNO}_{3}\left(\mathrm{H}_{2} \mathrm{O}\right)_{0.4}: \mathrm{C}, 70.7 ; \mathrm{H}, 5.7 ; \mathrm{N}, 2.65$. Found: C, 70.72; H, 5.77; N, 2.42.

N-\{5-ferrocenyl-6-(4-hydroxyphenyl)-6-phenyl-hex-5-enyl $\}$ succinimide (17). This compound was synthesized using the general procedure for imides starting from chloroalkene ferrociphenol 9 (1.3 g, $2.76 \mathrm{mmol})$, succinimide $(0.547 \mathrm{~g}, 5.5 \mathrm{mmol})$, potassium carbonate $(0.763 \mathrm{~g}, 5.5 \mathrm{mmol})$, DMF (70 mL) to afford 17 in a yield of 59\%. ${ }^{1} \mathrm{H}$ NMR (300 MHz, acetone- $\left.d_{6}\right): \delta=1.36-1.52\left(\mathrm{~m}, 4 \mathrm{H}, \mathrm{CH}_{2}-\mathrm{CH}_{2}\right), 2.55-2.72\left(\mathrm{~m}, 6 \mathrm{H}, 2 \mathrm{CH}_{2}\right.$ succ $+\mathrm{CH}_{2^{-}}$ $\mathrm{C}=\mathrm{C}), 3.23-3.36\left(\mathrm{~m}, 2 \mathrm{H}, \mathrm{CH}_{2} \mathrm{~N}\right), 3.86$ and $3.93\left(\mathrm{t}, J=1.9 \mathrm{~Hz}, 2 \mathrm{H}, \mathrm{C}_{5} \mathrm{H}_{4}\right), 4.04$ and $4.08\left(\mathrm{t}, J=1.9 \mathrm{~Hz}, 2 \mathrm{H}, \mathrm{C}_{5} \mathrm{H}_{4}\right)$, 4.13 and $4.14(\mathrm{~s}, 5 \mathrm{H}, \mathrm{Cp}), 6.72$ and $6.82\left(\mathrm{~d}, J=8.5 \mathrm{~Hz}, 2 \mathrm{H}, \mathrm{C}_{6} \mathrm{H}_{4}\right), 6.89$ and $7.05\left(\mathrm{~d}, J=8.5 \mathrm{~Hz}, 2 \mathrm{H}, \mathrm{C}_{6} \mathrm{H}_{4}\right), 7.05-$ $7.37(\mathrm{~m}, 5 \mathrm{H}, \mathrm{Ph}), 8.25$ and $8.35(\mathrm{~s}, 1 \mathrm{H}, \mathrm{OH}) .{ }^{13} \mathrm{C}$ NMR $\left(75 \mathrm{MHz}\right.$, acetone- $\left.d_{6}\right): \delta=28.5\left(\mathrm{CH}_{2}\right), 28.7\left(\mathrm{CH}_{2}\right), 28.8$ (2xCH 2, succ), $35.0\left(\mathrm{CH}_{2}\right), 38.8\left(\mathrm{CH}_{2}\right), 68.9\left(2 \mathrm{xCH}, \mathrm{C}_{5} \mathrm{H}_{4}\right), 70.0(5 \mathrm{xCH}, \mathrm{Cp}), 70.2\left(2 \mathrm{xCH}, \mathrm{C}_{5} \mathrm{H}_{4}\right), 88.0\left(\mathrm{C}, \mathrm{C}_{5} \mathrm{H}_{4}\right)$, $116.0\left(2 \mathrm{xCH}, \mathrm{C}_{6} \mathrm{H}_{4}\right), 127.0(\mathrm{CH}, \mathrm{Ph}), 129.1\left(2 \mathrm{xCH}_{\text {arom }}\right), 130.2\left(2 \mathrm{xCH}_{\text {arom }}\right), 131.8\left(2 \mathrm{xCH}_{\text {arom }}\right), 135.8(\mathrm{C}), 137.0$ (C), $139.4(\mathrm{C}), 146.0(\mathrm{C}), 156.9$ (C), 178.0 (2xC, C=O). IR (KBr, $\left.v \mathrm{~cm}^{-1}\right)$ : 1601 (CO). HRMS (ESI, $\mathrm{C}_{32} \mathrm{H}_{31} \mathrm{FeNO}_{3}$ : $[\mathrm{M}]^{+}$) calcd: 533.1648, found: 533.1645. Anal. Calcd for $\mathrm{C}_{32} \mathrm{H}_{31} \mathrm{FeNO}_{3}: \mathrm{C}, 72.05 ; \mathrm{H}, 5.85 ; \mathrm{N}, 2.62$. Found: C, 72.08; H, 5.97; N, 2.47 .

\section{N-\{4-ferrocenyl-5-(4-hydroxyphenyl)-5-phenyl-pent-4-enyl\}glutarimide (18). This compound was}

synthesized using the general procedure for imides starting from chloroalkene ferrociphenol $\mathbf{8}(0.566 \mathrm{~g}, 1.20$ mmol), glutarimide $(0.271 \mathrm{~g}, 2.40 \mathrm{mmol})$, potassium carbonate $(0.332 \mathrm{~g}, 2.40 \mathrm{mmol}), \mathrm{DMF}$ (40 mL) to afford 18 in a yield of $51 \%$. ${ }^{1} \mathrm{H}$ NMR (300 MHz, acetone- $\left.d_{6}\right): \delta=1.60-1.75\left(\mathrm{~m}, 2 \mathrm{H}, \mathrm{CH}_{2}\right), 1.79-1.93\left(\mathrm{~m}, 2 \mathrm{H}, \mathrm{CH}_{2}\right), 2.46-$ $2.61\left(\mathrm{~m}, 6 \mathrm{H}, \mathrm{CH}_{2}\right.$ glutarimide $\left.+\mathrm{CH}_{2}\right), 3.56-3.69\left(\mathrm{~m}, 2 \mathrm{H}, \mathrm{CH}_{2} \mathrm{~N}\right), 3.90-4.00\left(\mathrm{~m}, 2 \mathrm{H}, \mathrm{C}_{5} \mathrm{H}_{4}\right)$, 4.04-4.19 (m, 7H, $\left.\mathrm{C}_{5} \mathrm{H}_{4}+\mathrm{Cp}\right), 6.73$ and $6.81\left(\mathrm{~d}, J=8.3 \mathrm{~Hz}, 2 \mathrm{H}, \mathrm{C}_{6} \mathrm{H}_{4}\right), 6.92$ and $7.02\left(\mathrm{~d}, J=8.3 \mathrm{~Hz}, 2 \mathrm{H}, \mathrm{C}_{6} \mathrm{H}_{4}\right), 7.05-7.39(\mathrm{~m}, 5 \mathrm{H}$, $\mathrm{Ph}), 7.97(\mathrm{~s}, 1 \mathrm{H}, \mathrm{OH}) .{ }^{13} \mathrm{C} \mathrm{NMR}\left(75 \mathrm{MHz}\right.$, acetone- $\left.d_{6}\right): \delta=17.8\left(\mathrm{CH}_{2}\right.$, glutarimide), $30.1\left(\mathrm{CH}_{2}\right), 33.0\left(\mathrm{CH}_{2}\right), 33.2$ (2xCH 2 , glutarimide), $39.6\left(\mathrm{CH}_{2}\right), 68.8\left(2 \mathrm{xCH}, \mathrm{C}_{5} \mathrm{H}_{4}\right), 70.0(5 \mathrm{xCH}, \mathrm{Cp}), 70.1\left(2 \mathrm{xCH}, \mathrm{C}_{5} \mathrm{H}_{4}\right), 87.7\left(\mathrm{C}, \mathrm{C}_{5} \mathrm{H}_{4}\right)$, 115.9 and $116.0\left(2 \times \mathrm{XH}, \mathrm{C}_{6} \mathrm{H}_{4}\right), 126.9(\mathrm{CH}, \mathrm{Ph}), 128.9$ and $129.1\left(2 \mathrm{xCH}_{\text {arom }}\right), 130.1$ and $130.5\left(2 \mathrm{xCH}_{\text {arom }}\right), 131.2$ 
and $131.7\left(2 \mathrm{xCH}_{\text {arom }}\right), 135.2$ and $135.6(\mathrm{C}), 136.6$ and $136.8(\mathrm{C}), 139.3(\mathrm{C}), 145.8$ and $146.1(\mathrm{C}), 156.7$ and 156.8 (C), 173.0 (2xC, C=O). IR ( $\left.\mathrm{KBr}, v \mathrm{~cm}^{-1}\right)$ : 1658 (CO). HRMS (ESI, $\mathrm{C}_{32} \mathrm{H}_{31} \mathrm{FeNO}_{3}$ : [M] $]^{+}$) calcd: 533.1648, found: 533.1645. Anal. Calcd for $\mathrm{C}_{32} \mathrm{H}_{31} \mathrm{FeNO}_{3}\left(\mathrm{H}_{2} \mathrm{O}\right)_{0.1}$ : C, 71.81; H, 5.87; N, 2.61. Found: C, 72.17; H, 5.91; N, 2.21.

N-\{5-ferrocenyl-6-(4-hydroxyphenyl)-6-phenyl-hex-5-enyl\}glutarimide (19). This compound was synthesized using the general procedure for imides starting from chloroalkene ferrociphenol $\mathbf{9}(1 \mathrm{~g}, 2.12 \mathrm{mmol})$, glutarimide $(0.48 \mathrm{~g}, 4.2 \mathrm{mmol})$, potassium carbonate $(0.587 \mathrm{~g}, 4.2 \mathrm{mmol})$, DMF $(100 \mathrm{~mL})$ to afford 19 in a yield of $94 \%$. ${ }^{1} \mathrm{H}$ NMR (300 MHz, acetone- $\left.d_{6}\right): \delta=1.33-1.52\left(\mathrm{~m}, 4 \mathrm{H}, \mathrm{CH}_{2}-\mathrm{CH}_{2}\right), 1.87-1.96\left(\mathrm{~m}, 2 \mathrm{H}, \mathrm{CH}_{2}\right), 2.57-2.66\left(\mathrm{~m}, 6 \mathrm{H}, \mathrm{CH}_{2}\right.$ glutarimide $\left.+\mathrm{CH}_{2}\right), 3.59$ and $3.64\left(\mathrm{t}, J=6.8 \mathrm{~Hz}, 2 \mathrm{H}, \mathrm{CH}_{2} \mathrm{~N}\right), 3.87$ and $3.94\left(\mathrm{t}, J=1.9 \mathrm{~Hz}, 2 \mathrm{H}, \mathrm{C}_{5} \mathrm{H}_{4}\right), 4.06$ and $4.10\left(\mathrm{t}, J=1.9 \mathrm{~Hz}, 2 \mathrm{H}, \mathrm{C}_{5} \mathrm{H}_{4}\right), 4.15$ and $4.16(\mathrm{~s}, 5 \mathrm{H}, \mathrm{Cp}), 6.74$ and $6.83\left(\mathrm{~d}, J=8.6 \mathrm{~Hz}, 2 \mathrm{H}, \mathrm{C}_{6} \mathrm{H}_{4}\right), 6.91$ and 7.09 $\left(\mathrm{d}, J=8.6 \mathrm{~Hz}, 2 \mathrm{H}, \mathrm{C}_{6} \mathrm{H}_{4}\right), 7.05-7.39(\mathrm{~m}, 5 \mathrm{H}, \mathrm{Ph}), 8.21$ and $8.24(\mathrm{~s}, 1 \mathrm{H}, \mathrm{OH}) .{ }^{13} \mathrm{C}$ NMR $\left(75 \mathrm{MHz}\right.$, acetone- $\left.d_{6}\right): \delta=$ $17.9\left(\mathrm{CH}_{2}\right.$, glutarimide), 28.8 and $28.9\left(\mathrm{CH}_{2}\right), 29.0\left(\mathrm{CH}_{2}\right), 33.3\left(2 \mathrm{xCH}_{2}\right.$, glutarimide), 35.1 and $35.2\left(\mathrm{CH}_{2}\right), 39.4$ and $39.5\left(\mathrm{CH}_{2}\right), 68.8\left(2 \times \mathrm{CH}, \mathrm{C}_{5} \mathrm{H}_{4}\right), 69.90$ and $69.93(5 \mathrm{xCH}, \mathrm{Cp}), 70.0$ and $70.1\left(2 \mathrm{xCH}, \mathrm{C}_{5} \mathrm{H}_{4}\right), 87.89$ and 87.94 $\left(\mathrm{C}, \mathrm{C}_{5} \mathrm{H}_{4}\right), 115.9$ and $116.0\left(2 \mathrm{xCH}, \mathrm{C}_{6} \mathrm{H}_{4}\right), 126.8$ and $126.9(2 \mathrm{xCH}, \mathrm{Ph}), 128.9$ and $129.0\left(2 \mathrm{xCH}_{\text {arom }}\right), 130.1$ and $130.6\left(2 \mathrm{xCH}_{\text {arom }}\right), 131.2$ and $131.7\left(2 \mathrm{xCH}_{\text {arom }}\right), 135.8$ and $136.1(\mathrm{C}), 136.7$ and $137.0(\mathrm{C}), 139.2$ and $139.3(\mathrm{C})$, 146.0 and $146.3(\mathrm{C}), 156.7$ and $156.8(\mathrm{C}), 172.98$ and $173.0(2 \mathrm{xC}, \mathrm{C}=\mathrm{O})$. IR (KBr, $\left.v^{-1} \mathrm{~cm}^{-1}\right)$ 1649 (CO). HRMS (ESI, $\mathrm{C}_{33} \mathrm{H}_{33} \mathrm{FeNO}_{3}:[\mathrm{M}]^{+}$) calcd: 547.1804, found: 547.1801. Anal. Calcd for $\mathrm{C}_{33} \mathrm{H}_{33} \mathrm{FeNO}_{3}(\mathrm{AcOEt})_{0.4}: \mathrm{C}, 71.31$; H, 6.26; N, 2.4. Found: C, 71.49; H, 6.64; N, 2.08.

N-\{4-ferrocenyl-5-(4-hydroxyphenyl)-5-phenyl-pent-4-enyl\}phthalimide (20). This compound was synthesized using the general procedure for imides starting from chloroalkene ferrociphenol $\mathbf{8}(0.71 \mathrm{~g}, 1.55$ mmol), phthalimide (0.686 g, $4.7 \mathrm{mmol})$, potassium carbonate (0.644 g, $4.7 \mathrm{mmol})$, DMF (20 mL) to afford 20 in a yield of $75 \%$. ${ }^{1} \mathrm{H}$ NMR (300 MHz, acetone- $\left.d_{6}\right): \delta=1.82-1.97\left(\mathrm{~m}, 2 \mathrm{H}, \mathrm{CH}_{2}\right), 2.56-2.65$ and 2.65-2.74 (m, 2H, $\mathrm{CH}_{2}$ ), 3.56 and 3.60 (t, $\left.J=6.8 \mathrm{~Hz}, 2 \mathrm{H}, \mathrm{CH}_{2} \mathrm{~N}\right), 3.84$ and $3.93\left(\mathrm{t}, J=1.9 \mathrm{~Hz}, 2 \mathrm{H}, \mathrm{C}_{5} \mathrm{H}_{4}\right), 4.02$ and 4.06 (t, $J=1.9$ $\left.\mathrm{Hz}, 2 \mathrm{H}, \mathrm{C}_{5} \mathrm{H}_{4}\right), 4.07$ and $4.09(\mathrm{~s}, 5 \mathrm{H}, \mathrm{Cp}), 6.67$ and $6.70\left(\mathrm{~d}, J=8.5 \mathrm{~Hz}, 2 \mathrm{H}, \mathrm{C}_{6} \mathrm{H}_{4}\right), 6.88$ and $7.00(\mathrm{~d}, J=8.5 \mathrm{~Hz}$, $\left.2 \mathrm{H}, \mathrm{C}_{6} \mathrm{H}_{4}\right), 7.02-7.26(\mathrm{~m}, 5 \mathrm{H}, \mathrm{Ph}), 7.84\left(\mathrm{~s}, 4 \mathrm{H}\right.$, phthalimide), 8.11 and $8.25(\mathrm{~s}, 1 \mathrm{H}, \mathrm{OH}) .{ }^{13} \mathrm{C} \mathrm{NMR}(75 \mathrm{MHz}$, acetone- $\left.d_{6}\right): \delta=30.4$ and $30.7\left(\mathrm{CH}_{2}\right), 33.0\left(\mathrm{CH}_{2}\right), 38.4\left(\mathrm{CH}_{2}\right), 68.8\left(2 \mathrm{xCH}, \mathrm{C}_{5} \mathrm{H}_{4}\right), 69.9(5 \mathrm{xCH}, \mathrm{Cp}+2 \times \mathrm{xH}$, $\left.\mathrm{C}_{5} \mathrm{H}_{4}\right), 87.7$ and $87.8\left(\mathrm{C}, \mathrm{C}_{5} \mathrm{H}_{4}\right), 115.9\left(2 \times \mathrm{xH}_{1} \mathrm{C}_{6} \mathrm{H}_{4}\right), 123.6$ and $123.7(2 \times \mathrm{CH}$, phthalimide), 126.7 and $126.9(\mathrm{CH}$, $\mathrm{Ph}), 128.8$ and $128.9\left(2 \mathrm{xCH}_{\text {arom }}\right), 129.7$ and $130.5\left(2 \mathrm{xCH}_{\text {arom }}\right), 131.0$ and $131.6\left(2 \mathrm{xCH}_{\text {arom }}\right), 133.0$ and $133.1(\mathrm{C})$, 134.8 and 134.9 (2xCH, phthalimide), 135.0 and 135.2 (C), 136.2 and $136.6(\mathrm{C}), 139.6$ and 139.7 (C), 145.5 and 146.0 (C), 156.7 and 156.9 (C), 168.7 and 168.8 (2xC, C=O). IR (ATR, $\left.v \mathrm{~cm}^{-1}\right)$ : 1697 (CO). HRMS (ESI, $\mathrm{C}_{35} \mathrm{H}_{29} \mathrm{FeNO}_{3}:[\mathrm{M}+\mathrm{Na}]^{+}$) calcd: 567.149683, found: 567.1491. Anal. Calcd for $\mathrm{C}_{35} \mathrm{H}_{29} \mathrm{FeNO}_{3}\left(\mathrm{H}_{2} \mathrm{O}\right)_{0.5}: \mathrm{C}, 72.92$; H, 5.24; N, 2.42. Found: C, 72.99; H, 5.22; N, 2.25.

N-\{5-ferrocenyl-6-(4-hydroxyphenyl)-6-phenyl-hex-5-enyl $\}$ phthalimide (21). This compound was synthesized using the general procedure for imides starting from chloroalkene ferrociphenol 9 ( $1 \mathrm{~g}, 2.12 \mathrm{mmol})$, phthalimide 
(0.625 g, $4.2 \mathrm{mmol})$, potassium carbonate $(0.587 \mathrm{~g}, 4.2 \mathrm{mmol})$, DMF $(100 \mathrm{~mL})$ to afford 21 in a yield of $61 \%$. ${ }^{1} \mathrm{H}$ NMR (300 MHz, acetone- $\left.d_{6}\right): \delta=1.44-1.67\left(\mathrm{~m}, 4 \mathrm{H}, \mathrm{CH}_{2}-\mathrm{CH}_{2}\right), 2.64$ and $2.70\left(\mathrm{t}, J=7.7 \mathrm{~Hz}, 2 \mathrm{H}, \mathrm{CH}_{2}-\mathrm{C}=\mathrm{C}\right), 3.53$ and $3.57\left(\mathrm{t}, J=6.9 \mathrm{~Hz}, 2 \mathrm{H}, \mathrm{CH}_{2} \mathrm{~N}\right), 3.85$ and $3.92\left(\mathrm{t}, J=1.9 \mathrm{~Hz}, 2 \mathrm{H}, \mathrm{C}_{5} \mathrm{H}_{4}\right), 4.02$ and $4.05(\mathrm{t}, J=1.9 \mathrm{~Hz}, 2 \mathrm{H}$, $\left.\mathrm{C}_{5} \mathrm{H}_{4}\right), 4.11$ and $4.12(\mathrm{~s}, 5 \mathrm{H}, \mathrm{Cp}), 6.71\left(\mathrm{~d}, J=8.5 \mathrm{~Hz}, 2 \mathrm{H}, \mathrm{C}_{6} \mathrm{H}_{4}\right), 6.87$ and $7.01\left(\mathrm{~d}, J=8.5 \mathrm{~Hz}, 2 \mathrm{H}, \mathrm{C}_{6} \mathrm{H}_{4}\right), 7.02-$ $7.24(\mathrm{~m}, 5 \mathrm{H}, \mathrm{Ph}), 7.85$ (s, 4H, phthalimide), 8.17 and 8.19 (s, 1H, OH). ${ }^{13} \mathrm{C}$ NMR $\left(75 \mathrm{MHz}\right.$, acetone- $\left.d_{6}\right): \delta=28.5$ $\left(\mathrm{CH}_{2}\right), 29.2\left(\mathrm{CH}_{2}\right), 34.9\left(\mathrm{CH}_{2}\right), 38.2$ and $38.3\left(\mathrm{CH}_{2}\right), 68.7\left(2 \mathrm{xCH}, \mathrm{C}_{5} \mathrm{H}_{4}\right), 69.8$ and $69.9(5 \times C H, \mathrm{Cp}), 70.0$ and 70.1 (2xCH, $\left.\mathrm{C}_{5} \mathrm{H}_{4}\right), 87.9$ and $88.0\left(\mathrm{C}, \mathrm{C}_{5} \mathrm{H}_{4}\right), 115.8$ and $115.9\left(2 \mathrm{xCH}, \mathrm{C}_{6} \mathrm{H}_{4}\right), 123.6(2 \mathrm{xCH}$, phthalimide), $126.8(\mathrm{CH}$, $\mathrm{Ph}), 128.8$ and $128.9\left(2 \mathrm{xCH}_{\text {arom }}\right), 129.9$ and $130.6\left(2 \mathrm{xCH}_{\text {arom }}\right), 131.1$ and $131.7\left(2 \mathrm{xCH}_{\text {arom }}\right), 133.1(2 \mathrm{xC}), 134.6$ (2xCH, phthalimide), 135.6 and 135.9 (C), 136.6 and 136.8 (C), 139.3 and $139.4(\mathrm{C}), 145.8$ and 146.2 (C), 156.6 and $156.8(\mathrm{C}), 168.6$ and $168.7(2 \mathrm{xC}, \mathrm{C}=\mathrm{O})$. IR (KBr, $\left.v \mathrm{~cm}^{-1}\right)$ : $1711(\mathrm{CO})$. HRMS (ESI, $\mathrm{C}_{36} \mathrm{H}_{31} \mathrm{FeNO}_{3}:[\mathrm{M}]^{+}$) calcd: 581.1648, found: 581.1646.

General procedure for the chemical oxidation of imodo-ferrociphenols to quinone methides. Freshly made $\mathrm{Ag}_{2} \mathrm{O}^{1}$ was added to a solution of imodo-ferrociphenols in acetone. The dark grey mixture obtained changed to be dark red suspension about 5 minutes later. The reaction was monitored by TLC until complete conversion of the starting material (around 40 minutes). Filtration was followed by removal of the solvent under reduced pressure to give quinone methides as a dark red solid.

Quinone methide 4-QM. Following the general procedure for the oxidation, $\operatorname{Ag}_{2} \mathrm{O}(0.2 \mathrm{~g}, 0.9$ mmoles $)$, compound 4 (0.08 g, 0.15 mmoles). Compound 4-QM was obtained as a dark red solid $75 \mathrm{mg}$, yield $93 \%$. ${ }^{1} \mathrm{H}$ NMR (400 MHz, acetone- $\left.d_{6}\right): \delta=2.27\left(\mathrm{q}, J=7.0 \mathrm{~Hz}, 2 \mathrm{H}, \mathrm{CH}_{2}\right), 2.65\left(\mathrm{~s}, 4 \mathrm{H}, 2 \mathrm{xCH}_{2}\right), 3.57(\mathrm{t}, J=6.9 \mathrm{~Hz}, 2 \mathrm{H}$, $\mathrm{CH}_{2}$ ), 4.02 (s, 5H, 5xCH; Cp), $4.14-4.25$ (m, 4H, C $\left.\mathrm{H}_{4}\right), 6.34$ (m, J= 7.5 Hz, 3H, 2xCH+CH), 6.97 (br s, 2H; $\left.\mathrm{C}_{6} \mathrm{H}_{4}\right), 7.39$ (br s, $2 \mathrm{H} ; \mathrm{C}_{6} \mathrm{H}_{4}$ ), 7.54 (br s, $\left.2 \mathrm{H} ; \mathrm{C}_{6} \mathrm{H}_{4}\right), 9.03$ (s, $\left.1 \mathrm{H}, \mathrm{OH}\right) .{ }^{13} \mathrm{C}$ NMR $\left(100 \mathrm{MHz}\right.$, acetone-d $\left.d_{6}\right): \delta=28.0$ $\left(\mathrm{CH}_{2}\right), 28.6\left(2 \mathrm{xCH}_{2}\right), 37.4\left(\mathrm{CH}_{2}\right), 67.0\left(2 \mathrm{xCH}, \mathrm{C}_{5} \mathrm{H}_{4}\right), 68.1\left(2 \mathrm{xCH}, \mathrm{C}_{5} \mathrm{H}_{4}\right), 69.2(5 \mathrm{xCH}, \mathrm{Cp}), 87.1(\mathrm{C}), 115.4$ (2xCH, $\left.\mathrm{C}_{6} \mathrm{H}_{4}\right), 126.7(\mathrm{CH}), 127.8(\mathrm{C}), 128.6(\mathrm{C}), 128.7\left(\mathrm{CH} ; \mathrm{C}_{6} \mathrm{H}_{4}\right), 132.3$ (2xCH; $\left.\mathrm{C}_{6} \mathrm{H}_{4}\right), 133.0(\mathrm{C}), 137.9(\mathrm{C})$, $138.9(\mathrm{CH}), 139.4\left(2 \times \mathrm{CH} ; \mathrm{C}_{6} \mathrm{H}_{4}\right), 157.1(\mathrm{C}), 177.1(2 \mathrm{C}, \mathrm{C}=\mathrm{O}), 185.9(\mathrm{C}, \mathrm{C}=\mathrm{O})$. HRMS calcd for $\mathrm{C}_{31} \mathrm{H}_{27} \mathrm{FeO}_{4}$ $(\mathrm{M})^{+}:$533.1290, found: 533.1284 .

Quinone methide 16-QM. Following the general procedure for the oxidation, $\mathrm{Ag}_{2} \mathrm{O}$ (60 mg, $\left.0.3 \mathrm{mmoles}\right)$, compound 16 (25 mg, 0.05 mmoles). Compound 16-QM was obtained as a dark red solid $22 \mathrm{mg}$, yield 84\%. ${ }^{1} \mathrm{H}$ NMR (400 MHz, acetone- $\left.d_{6}\right): \delta=2.34\left(\mathrm{q}, J=7.1 \mathrm{~Hz}, 2 \mathrm{H}, \mathrm{CH}_{2}\right), 2.68\left(\mathrm{~s}, 4 \mathrm{H}, 2 \mathrm{xCH}_{2}\right), 3.61(\mathrm{t}, J=6.9 \mathrm{~Hz}, 2 \mathrm{H}$, $\mathrm{CH}_{2}$ ), 4.00 (s, 5H, 5xCH; Cp), $4.11\left(\mathrm{~s}, 1 \mathrm{H}, \mathrm{C}_{5} \mathrm{H}_{4}\right), 4.14\left(\mathrm{~s}, 1 \mathrm{H}, \mathrm{C}_{5} \mathrm{H}_{4}\right), 4.19\left(\mathrm{~s}, 1 \mathrm{H}, \mathrm{C}_{5} \mathrm{H}_{4}\right), 4.29\left(\mathrm{~s}, 1 \mathrm{H}, \mathrm{C}_{5} \mathrm{H}_{4}\right), 6.35$ $-6.45(\mathrm{~m}, J=7.5 \mathrm{~Hz}, 3 \mathrm{H}, 2 \mathrm{xCH}+\mathrm{CH}), 7.45-7.57\left(\mathrm{~m}, 6 \mathrm{H}, \mathrm{C}_{6} \mathrm{H}_{4}\right), 7.65\left(\mathrm{dd}, J=10.0,2.7 \mathrm{~Hz}, 1 \mathrm{H}, \mathrm{C}_{6} \mathrm{H}_{4}\right) .{ }^{13} \mathrm{C} \mathrm{NMR}$ $\left(100 \mathrm{MHz}\right.$, acetone- $\left.d_{6}\right): \delta=28.0\left(\mathrm{CH}_{2}\right), 28.7\left(2 \mathrm{xCH}_{2}\right), 37.4\left(\mathrm{CH}_{2}\right), 68.2\left(2 \mathrm{xCH}, \mathrm{C}_{5} \mathrm{H}_{4}\right), 69.2\left(2 \mathrm{xCH}, \mathrm{C}_{5} \mathrm{H}_{4}\right), 69.3$ (5xCH, Cp), $86.7(\mathrm{C}), 126.5(\mathrm{CH}), 128.4(\mathrm{CH}), 128.5(\mathrm{CH}), 128.9(\mathrm{CH}), 129.8(\mathrm{CH}), 129.9(\mathrm{C}), 130.7(\mathrm{CH})$, $131.1(\mathrm{CH}), 131.1(\mathrm{CH}), 137.4(\mathrm{CH}), 137.8(\mathrm{C}), 138.7(\mathrm{CH}), 139.2(\mathrm{C}), 156.3(\mathrm{C}), 177.4(2 \mathrm{C}, \mathrm{C}=\mathrm{O}), 186.0(\mathrm{C}$, $\mathrm{C}=\mathrm{O})$. HRMS calcd for $\mathrm{C}_{31} \mathrm{H}_{27} \mathrm{FeO}_{3}(\mathrm{M})^{+}:$517.1340, found: 517.1335. 
Quinone methide 10-QM. Following the general procedure for the oxidation, $\mathrm{Ag}_{2} \mathrm{O}(0.13 \mathrm{~g}, 0.5 \mathrm{mmoles})$, compound 10 (0.05 g, 0.09 mmoles). Compound 10-QM was obtained as a dark red solid $45 \mathrm{mg}$, yield $91 \%$. ${ }^{1} \mathrm{H}$ NMR $\left(400 \mathrm{MHz}\right.$, acetone- $\left.d_{6}\right): \delta=1.68\left(\mathrm{~m}, J=6.7 \mathrm{~Hz}, 2 \mathrm{H}, \mathrm{CH}_{2}\right), 1.99\left(\mathrm{~m}, 2 \mathrm{H}, \mathrm{CH}_{2}\right), 2.60\left(\mathrm{~s}, 4 \mathrm{H}, 2 \mathrm{xCH}_{2}\right), 3.42(\mathrm{t}$, $\left.J=6.9 \mathrm{~Hz}, 2 \mathrm{H}, \mathrm{CH}_{2}\right), 4.01(\mathrm{~s}, 5 \mathrm{H}, 5 \mathrm{xCH} ; \mathrm{Cp}), 4.10-4.20\left(\mathrm{~m}, 4 \mathrm{H}, \mathrm{C}_{5} \mathrm{H}_{4}\right), 6.39(\mathrm{~m}, J=7.4 \mathrm{~Hz}, 3 \mathrm{H}, 2 \mathrm{xCH}+\mathrm{CH})$, 6.99 (br s, $2 \mathrm{H} ; \mathrm{C}_{6} \mathrm{H}_{4}$ ), 7.42 (br s, $\left.2 \mathrm{H} ; \mathrm{C}_{6} \mathrm{H}_{4}\right), 7.58$ (br s, $\left.2 \mathrm{H} ; \mathrm{C}_{6} \mathrm{H}_{4}\right), 9.14$ (s, $\left.1 \mathrm{H}, \mathrm{OH}\right) .{ }^{13} \mathrm{C} \mathrm{NMR}(100 \mathrm{MHz}$, acetone- $\left.d_{6}\right): \delta=27.1\left(\mathrm{CH}_{2}\right), 27.2\left(2 \mathrm{xCH}_{2}\right), 37.7\left(\mathrm{CH}_{2}\right), 66.9\left(\mathrm{CH}, \mathrm{C}_{5} \mathrm{H}_{4}\right), 68.0\left(2 \mathrm{xCH}, \mathrm{C}_{5} \mathrm{H}_{4}\right), 69.1\left(\mathrm{CH}, \mathrm{C}_{5} \mathrm{H}_{4}\right)$, 69.2 (5xCH, Cp), $87.4(\mathrm{C}), 115.4\left(2 \mathrm{xCH}, \mathrm{C}_{6} \mathrm{H}_{4}\right), 128.6\left(2 \mathrm{xCH}, \mathrm{C}_{6} \mathrm{H}_{4}\right), 129.6\left(2 \mathrm{xCH}, \mathrm{C}_{6} \mathrm{H}_{4}\right), 133.2(\mathrm{C}), 136.1(\mathrm{C})$, $138.1\left(2 \mathrm{xCH}, \mathrm{C}_{6} \mathrm{H}_{4}\right), 157.8(\mathrm{C}), 177.1(2 \mathrm{C}, \mathrm{C}=\mathrm{O}), 186.1(\mathrm{C}, \mathrm{C}=\mathrm{O})$. HRMS calcd for $\mathrm{C}_{32} \mathrm{H}_{29} \mathrm{FeO}_{4}(\mathrm{M})^{+}$: 547.1446, found: 547.1441. 10-Indene. ${ }^{1} \mathrm{H}$ NMR (400 MHz, acetone- $\left.d_{6}\right): \delta=1.05\left(\mathrm{~m}, 1 \mathrm{H}, \mathrm{CH}_{2}\right), 1.25\left(\mathrm{~m}, 1 \mathrm{H}, \mathrm{CH}_{2}\right), 1.84$ (m, 1H, $\left.\mathrm{CH}_{2}\right), 2.07\left(\mathrm{~m}, 1 \mathrm{H}, \mathrm{CH}_{2}\right), 2.41\left(\mathrm{~s}, 4 \mathrm{H}, 2 \mathrm{xCH}_{2}\right), 3.19\left(\mathrm{~m}, 2 \mathrm{H}, \mathrm{CH}_{2}\right), 3.71(\mathrm{~m}, 1 \mathrm{H}, \mathrm{CH}), 3.87(\mathrm{~s}, 5 \mathrm{H}, 5 \mathrm{xCH}$; $\mathrm{Cp}), 3.91\left(\mathrm{~s}, 1 \mathrm{H}, \mathrm{C}_{5} \mathrm{H}_{4}\right), 3.98\left(\mathrm{~s}, 1 \mathrm{H}, \mathrm{C}_{5} \mathrm{H}_{4}\right), 4.09\left(\mathrm{~s}, 1 \mathrm{H}, \mathrm{C}_{5} \mathrm{H}_{4}\right), 4.29\left(\mathrm{~s}, 1 \mathrm{H}, \mathrm{C}_{5} \mathrm{H}_{4}\right), 6.57\left(\mathrm{~m}, 2 \mathrm{H}, \mathrm{C}_{6} \mathrm{H}_{3}\right), 6.82(\mathrm{~s}$, $\left.1 \mathrm{H}, \mathrm{C}_{6} \mathrm{H}_{3}\right), 6.90\left(\mathrm{~d}, J=8.5 \mathrm{~Hz} 2 \mathrm{H} ; \mathrm{C}_{6} \mathrm{H}_{4}\right), 7.10\left(\mathrm{~d}, J=8.5 \mathrm{~Hz} 2 \mathrm{H} ; \mathrm{C}_{6} \mathrm{H}_{4}\right), 8.09(\mathrm{~s}, 1 \mathrm{H}, \mathrm{OH}), 8.45(\mathrm{~s}, 1 \mathrm{H}, \mathrm{OH}) .{ }^{13} \mathrm{C}$ NMR (100 MHz, acetone- $\left.d_{6}\right): \delta=22.2\left(\mathrm{CH}_{2}\right), 27.8\left(2 \mathrm{xCH}_{2}\right), 29.2\left(\mathrm{CH}_{2}\right), 38.2\left(\mathrm{CH}_{2}\right), 49.8(\mathrm{CH}), 66.6\left(\mathrm{CH}, \mathrm{C}_{5} \mathrm{H}_{4}\right)$, $67.8\left(\mathrm{CH}, \mathrm{C}_{5} \mathrm{H}_{4}\right), 68.3\left(\mathrm{CH}, \mathrm{C}_{5} \mathrm{H}_{4}\right), 68.4\left(\mathrm{CH}, \mathrm{C}_{5} \mathrm{H}_{4}\right), 69.2(5 x \mathrm{CH}, \mathrm{Cp}), 81.0(\mathrm{C}), 110.6(\mathrm{CH}), 113.3(\mathrm{CH}), 115.4$ $\left(2 x \mathrm{xH}, \mathrm{C}_{6} \mathrm{H}_{4}\right), 119.6(\mathrm{CH}), 128.1(\mathrm{C}), 130.6\left(2 \times \mathrm{CH}_{1} \mathrm{C}_{6} \mathrm{H}_{4}\right), 138.4(\mathrm{C}), 139.6(\mathrm{C}), 148.3(\mathrm{C}), 155.4(\mathrm{C}), 156.8(\mathrm{C})$, $177.2(2 \mathrm{C}, \mathrm{C}=\mathrm{O})$.

Quinone methide 17-QM. Following the general procedure for the oxidation, $\mathrm{Ag}_{2} \mathrm{O}$ ( $\left.80 \mathrm{mg}, 0.4 \mathrm{mmoles}\right)$, compound 17 (40 mg, 0.08 mmoles). Compound 17-QM was obtained as a dark red solid $35 \mathrm{mg}$, yield $88 \%$. ${ }^{1} \mathrm{H}$ NMR (400 MHz, acetone- $\left.d_{6}\right): \delta=1.72\left(\mathrm{~m}, 2 \mathrm{H}, \mathrm{CH}_{2}\right), 2.09\left(\mathrm{~m}, 2 \mathrm{H}, \mathrm{CH}_{2}\right), 2.62\left(\mathrm{~s}, 4 \mathrm{H}, 2 \mathrm{xCH}_{2}\right), 3.46(\mathrm{t}, J=7.1 \mathrm{~Hz}$, $\left.2 \mathrm{H}, \mathrm{CH}_{2}\right), 4.00$ (s, 5H, 5xCH; Cp), $4.10\left(\mathrm{~s}, 1 \mathrm{H}, \mathrm{C}_{5} \mathrm{H}_{4}\right), 4.15\left(\mathrm{~s}, 1 \mathrm{H}, \mathrm{C}_{5} \mathrm{H}_{4}\right), 4.18\left(\mathrm{~s}, 1 \mathrm{H}, \mathrm{C}_{5} \mathrm{H}_{4}\right), 4.31\left(\mathrm{~s}, 1 \mathrm{H}, \mathrm{C}_{5} \mathrm{H}_{4}\right)$, $6.37-6.44(\mathrm{~m}, J=7.5 \mathrm{~Hz}, 3 \mathrm{H}, 2 \mathrm{xCH}+\mathrm{CH}), 7.46-7.57\left(\mathrm{~m}, 6 \mathrm{H}, \mathrm{C}_{6} \mathrm{H}_{4}\right), 7.65\left(\mathrm{dd}, J=10.2,2.7 \mathrm{~Hz}, 1 \mathrm{H}, \mathrm{C}_{6} \mathrm{H}_{4}\right) .{ }^{13} \mathrm{C}$ NMR (100 MHz, acetone- $\left.d_{6}\right): \delta=26.6\left(\mathrm{CH}_{2}\right), 27.3\left(\mathrm{CH}_{2}\right), 27.9\left(2 \mathrm{xCH}_{2}\right), 37.7\left(\mathrm{CH}_{2}\right), 66.7\left(2 \times C H, \mathrm{C}_{5} \mathrm{H}_{4}\right), 68.1$ (2xCH, $\left.\mathrm{C}_{5} \mathrm{H}_{4}\right), 69.3(5 \mathrm{xCH}, \mathrm{Cp}), 87.0(\mathrm{C}), 128.4\left(2 \mathrm{xCH}_{0} \mathrm{C}_{6} \mathrm{H}_{4}\right), 128.5(\mathrm{CH}), 128.9(\mathrm{C}), 129.4(\mathrm{CH}), 129.6(\mathrm{CH})$, $129.8(\mathrm{CH}), 130.8\left(2 \times \mathrm{CH}, \mathrm{C}_{6} \mathrm{H}_{4}\right), 137.4(\mathrm{CH}), 137.8(\mathrm{C}), 137.9(\mathrm{C}), 138.8(\mathrm{CH}), 156.7(\mathrm{C}), 177.1(2 \mathrm{C}, \mathrm{C}=\mathrm{O})$, $186.0(\mathrm{C}, \mathrm{C}=\mathrm{O})$. HRMS calcd for $\mathrm{C}_{32} \mathrm{H}_{29} \mathrm{FeO}_{3}(\mathrm{M})^{+}:$531.1497, found: 531.1491. 17-Indene. ${ }^{1} \mathrm{H}$ NMR (400 MHz, acetone- $\left.d_{6}\right): \delta=1.06\left(\mathrm{~m}, 1 \mathrm{H}, \mathrm{CH}_{2}\right), 1.26\left(\mathrm{~m}, 1 \mathrm{H}, \mathrm{CH}_{2}\right), 1.86\left(\mathrm{~m}, 1 \mathrm{H}, \mathrm{CH}_{2}\right), 2.09\left(\mathrm{~m}, 1 \mathrm{H}, \mathrm{CH}_{2}\right), 2.41(\mathrm{~s}, 4 \mathrm{H}$, $\left.2 \mathrm{xCH}_{2}\right), 3.18\left(\mathrm{~m}, 2 \mathrm{H}, \mathrm{CH}_{2}\right), 3.74(\mathrm{~m}, 1 \mathrm{H}, \mathrm{CH}), 3.84\left(\mathrm{~s}, 1 \mathrm{H}, \mathrm{C}_{5} \mathrm{H}_{4}\right), 3.87(\mathrm{~s}, 5 \mathrm{H}, 5 \mathrm{xCH} ; \mathrm{Cp}), 3.99\left(\mathrm{~s}, 1 \mathrm{H}, \mathrm{C}_{5} \mathrm{H}_{4}\right)$, $4.11\left(\mathrm{~s}, 1 \mathrm{H}, \mathrm{C}_{5} \mathrm{H}_{4}\right), 4.31\left(\mathrm{~s}, 1 \mathrm{H}, \mathrm{C}_{5} \mathrm{H}_{4}\right), 6.53\left(\mathrm{~m}, 2 \mathrm{H}, \mathrm{C}_{6} \mathrm{H}_{3}\right), 6.84\left(\mathrm{~s}, 1 \mathrm{H}, \mathrm{C}_{6} \mathrm{H}_{3}\right), 7.28\left(\mathrm{~m}, 2 \mathrm{H}, \mathrm{C}_{6} \mathrm{H}_{5}\right), 7.34(\mathrm{~d}, J=$ $\left.7.5 \mathrm{~Hz}, 1 \mathrm{H} ; \mathrm{C}_{6} \mathrm{H}_{5}\right), 7.42\left(\mathrm{~d}, J=7.6 \mathrm{~Hz}, 2 \mathrm{H} ; \mathrm{C}_{6} \mathrm{H}_{5}\right), 8.06(\mathrm{~s}, 1 \mathrm{H}, \mathrm{OH}) .{ }^{13} \mathrm{C}$ NMR $\left(100 \mathrm{MHz}\right.$, acetone- $\left.d_{6}\right): \delta=22.2$ $\left(\mathrm{CH}_{2}\right), 26.6\left(\mathrm{CH}_{2}\right), 27.8\left(2 \mathrm{xCH}_{2}\right), 38.2\left(\mathrm{CH}_{2}\right), 50.0(\mathrm{CH}), 66.7\left(\mathrm{CH}, \mathrm{C}_{5} \mathrm{H}_{4}\right), 68.0\left(\mathrm{CH}, \mathrm{C}_{5} \mathrm{H}_{4}\right), 68.2\left(\mathrm{CH}, \mathrm{C}_{5} \mathrm{H}_{4}\right)$, $68.6\left(\mathrm{CH}, \mathrm{C}_{5} \mathrm{H}_{4}\right), 69.3(5 \times \mathrm{CH}, \mathrm{Cp}), 80.8(\mathrm{C}), 110.6(\mathrm{CH}), 113.4(\mathrm{CH}), 119.5(\mathrm{CH}), 127.3(\mathrm{CH}), 128.5(\mathrm{CH}), 128.6$ (2xCH, $\left.\mathrm{C}_{6} \mathrm{H}_{5}\right), 129.5\left(2 \mathrm{xCH}_{0} \mathrm{C}_{6} \mathrm{H}_{5}\right), 137.4(\mathrm{C}), 138.4(\mathrm{C}), 139.2(\mathrm{C}), 140.1(\mathrm{C}), 148.4(\mathrm{C}), 155.6(\mathrm{C}), 177.2$ (2C, $\mathrm{C}=\mathrm{O})$.

Quinone methide 12-QM. Following the general procedure for the oxidation, $\mathrm{Ag}_{2} \mathrm{O}$ (0.18 g, $\left.0.8 \mathrm{mmoles}\right)$, compound 12 (0.08 g, 0.14 mmoles). Compound 12-QM was obtained as a dark red solid $73 \mathrm{mg}$, yield $95 \%$. ${ }^{1} \mathrm{H}$ NMR (400 MHz, acetone- $\left.d_{6}\right): \delta=1.92(\mathrm{~m}, 2 \mathrm{H}), 2.22\left(\mathrm{q}, J=7.2 \mathrm{~Hz}, 2 \mathrm{H}, \mathrm{CH}_{2}\right), 2.62\left(\mathrm{t}, J=6.2 \mathrm{~Hz}, 4 \mathrm{H}, 2 \mathrm{xCH}_{2}\right)$, 
$3.84\left(\mathrm{t}, J=7.1 \mathrm{~Hz}, 2 \mathrm{H}, \mathrm{CH}_{2}\right), 4.00(\mathrm{~s}, 5 \mathrm{H}, 5 \mathrm{xCH} ; \mathrm{Cp}), 4.13-4.23\left(\mathrm{~m}, 4 \mathrm{H}, \mathrm{C}_{5} \mathrm{H}_{4}\right), 6.31(\mathrm{t}, J=7.5 \mathrm{~Hz}, 1 \mathrm{H}, \mathrm{CH})$, 6.68 (br s, 4H; $\left.\mathrm{C}_{6} \mathrm{H}_{4}\right), 7.49$ (d, $\left.J=7.8 \mathrm{~Hz}, 4 \mathrm{H} ; \mathrm{C}_{6} \mathrm{H}_{4}\right), 9.16$ (br s, $\left.1 \mathrm{H}, \mathrm{OH}\right) .{ }^{13} \mathrm{C}$ NMR $\left(100 \mathrm{MHz}\right.$, acetone- $\left.d_{6}\right): \delta=$ $17.0\left(\mathrm{CH}_{2}\right), 28.4\left(\mathrm{CH}_{2}\right), 32.4\left(2 \mathrm{xCH}_{2}\right), 38.1\left(\mathrm{CH}_{2}\right), 66.9\left(2 \mathrm{xCH}, \mathrm{C}_{5} \mathrm{H}_{4}\right), 68.0\left(2 \mathrm{xCH}, \mathrm{C}_{5} \mathrm{H}_{4}\right), 69.3(5 x \mathrm{CH}, \mathrm{Cp}), 87.2$ (C), $114.5\left(\mathrm{CH}, \mathrm{C}_{6} \mathrm{H}_{4}\right), 115.2\left(\mathrm{CH}, \mathrm{C}_{6} \mathrm{H}_{4}\right), 127.0\left(2 \times \mathrm{xH}_{1} \mathrm{C}_{6} \mathrm{H}_{4}\right), 127.9\left(\mathrm{CH}, \mathrm{C}_{6} \mathrm{H}_{4}\right), 128.3(\mathrm{C}), 128.8(\mathrm{CH}), 130.6$ (C), $133.0\left(\mathrm{CH}, \mathrm{C}_{6} \mathrm{H}_{4}\right), 137.8(\mathrm{C}), 138.9\left(2 \times \mathrm{CH}, \mathrm{C}_{6} \mathrm{H}_{4}\right), 139.1(\mathrm{C}), 157.4(\mathrm{C}), 172.3(2 \mathrm{C}, \mathrm{C}=\mathrm{O}), 185.9(\mathrm{C}, \mathrm{C}=\mathrm{O})$. HRMS calcd for $\mathrm{C}_{32} \mathrm{H}_{29} \mathrm{FeO}_{4}(\mathrm{M})^{+}$: 547.1446, found: 547.1441.

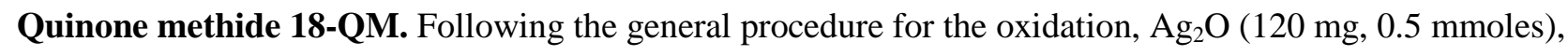
compound 18 (50 mg, 0.1 mmoles). Compound 18-QM was obtained as a dark red solid $45 \mathrm{mg}$, yield 90\%. ${ }^{1} \mathrm{H}$ NMR (400 MHz, acetone- $\left.d_{6}\right): \delta=1.94\left(\mathrm{~m}, 2 \mathrm{H}, \mathrm{CH}_{2}\right), 2.29\left(\mathrm{q}, J=7.6 \mathrm{~Hz}, 2 \mathrm{H}, \mathrm{CH}_{2}\right), 2.64\left(\mathrm{~m}, 4 \mathrm{H}, 2 \mathrm{xCH}_{2}\right), 3.89$ $\left(\mathrm{td}, J=6.8,3.1 \mathrm{~Hz}, 2 \mathrm{H}, \mathrm{CH}_{2}\right), 3.99(\mathrm{~s}, 5 \mathrm{H}, 5 \mathrm{xCH} ; \mathrm{Cp}), 4.13\left(\mathrm{~m}, 2 \mathrm{H}, \mathrm{C}_{5} \mathrm{H}_{4}\right), 4.18\left(\mathrm{~s}, 1 \mathrm{H}, \mathrm{C}_{5} \mathrm{H}_{4}\right), 4.28\left(\mathrm{~s}, 1 \mathrm{H}, \mathrm{C}_{5} \mathrm{H}_{4}\right)$, $6.31-6.46(\mathrm{~m}, 3 \mathrm{H}, 2 \mathrm{xCH}+\mathrm{CH}), 7.43-7.61\left(\mathrm{~m}, 6 \mathrm{H}, \mathrm{C}_{6} \mathrm{H}_{4}\right), 7.66\left(\mathrm{dd}, J=10.0,2.7 \mathrm{~Hz}, 1 \mathrm{H}, \mathrm{C}_{6} \mathrm{H}_{4}\right) .{ }^{13} \mathrm{C} \mathrm{NMR}(100$ MHz, acetone- $\left.d_{6}\right): \delta=17.0\left(\mathrm{CH}_{2}\right), 26.6\left(2 \mathrm{xCH}_{2}\right), 32.4\left(\mathrm{CH}_{2}\right), 38.1\left(\mathrm{CH}_{2}\right), 68.1\left(2 \times C H, \mathrm{C}_{5} \mathrm{H}_{4}\right), 69.2\left(2 \times \mathrm{CH}_{1} \mathrm{C}_{5} \mathrm{H}_{4}\right)$, $69.3(5 x \mathrm{xH}, \mathrm{Cp}), 86.7(\mathrm{C}), 126.7(\mathrm{CH}), 128.2(\mathrm{C}), 128.4\left(2 \times \mathrm{CH}, \mathrm{C}_{6} \mathrm{H}_{4}\right), 128.5(\mathrm{CH}), 128.9(\mathrm{CH}), 129.7(\mathrm{CH})$, $129.9(\mathrm{CH}), 130.7\left(2 \times \mathrm{CH}, \mathrm{C}_{6} \mathrm{H}_{4}\right), 131.1(\mathrm{C}), 137.3(\mathrm{CH}), 137.8(\mathrm{C}), 138.7(\mathrm{C}), 138.8(\mathrm{CH}), 156.3(\mathrm{C}), 172.3(2 \mathrm{C}$, $\mathrm{C}=\mathrm{O}), 185.9(\mathrm{C}, \mathrm{C}=\mathrm{O})$. HRMS calcd for $\mathrm{C}_{32} \mathrm{H}_{29} \mathrm{FeO}_{3}(\mathrm{M})^{+}:$531.1497, found: 531.1491.

Quinone methide 13-QM. Following the general procedure for the oxidation, $\mathrm{Ag}_{2} \mathrm{O}$ ( $\left.0.05 \mathrm{~g}, 0.3 \mathrm{mmoles}\right)$, compound 13 (0.03 g, 0.05 mmoles). Compound 13-QM was obtained as a dark red solid $25 \mathrm{mg}$, yield $89 \%$. ${ }^{1} \mathrm{H}$ NMR (400 MHz, acetone- $\left.d_{6}\right): \delta=1.65\left(\mathrm{~m}, J=7.5 \mathrm{~Hz}, 2 \mathrm{H}, \mathrm{CH}_{2}\right), 1.88\left(\mathrm{~m}, J=6.1 \mathrm{~Hz}, 2 \mathrm{H}, \mathrm{CH}_{2}\right), 2.59(\mathrm{t}, J=6.5$ $\left.\mathrm{Hz}, 4 \mathrm{H}, 2 \mathrm{xCH}_{2}\right), 3.72\left(\mathrm{t}, J=6.9 \mathrm{~Hz}, 2 \mathrm{H}, \mathrm{CH}_{2}\right), 4.03(\mathrm{~s}, 5 \mathrm{H}, 5 \mathrm{xCH} ; \mathrm{Cp}), 4.10-4.20\left(\mathrm{~m}, 4 \mathrm{H}, \mathrm{C}_{5} \mathrm{H}_{4}\right), 6.40(\mathrm{~m}, 3 \mathrm{H}$, $2 \mathrm{xCH}+\mathrm{CH}), 7.00\left(\mathrm{~d}, J=8.4 \mathrm{~Hz}, 2 \mathrm{H} ; \mathrm{C}_{6} \mathrm{H}_{4}\right), 7.41\left(\mathrm{~d}, J=8.0 \mathrm{~Hz}, 2 \mathrm{H} ; \mathrm{C}_{6} \mathrm{H}_{4}\right), 7.58\left(\mathrm{~d}, J=10.9 \mathrm{~Hz}, 1 \mathrm{H} ; \mathrm{C}_{6} \mathrm{H}_{4}\right), 7.64$ $\left(\mathrm{d}, J=10.2 \mathrm{~Hz}, 1 \mathrm{H} ; \mathrm{C}_{6} \mathrm{H}_{4}\right), 9.07$ (s, $\left.1 \mathrm{H}, \mathrm{OH}\right) .{ }^{13} \mathrm{C}$ NMR (100 MHz, acetone- $\left.d_{6}\right): \delta=17.0\left(\mathrm{CH}_{2}\right), 26.7\left(\mathrm{CH}_{2}\right), 27.5$ $\left(\mathrm{CH}_{2}\right), 32.3\left(2 \mathrm{xCH}_{2}\right), 38.3\left(\mathrm{CH}_{2}\right), 66.7\left(2 \mathrm{xCH}, \mathrm{C}_{5} \mathrm{H}_{4}\right), 68.0\left(2 \mathrm{xCH}, \mathrm{C}_{5} \mathrm{H}_{4}\right), 69.2(5 \mathrm{xCH}, \mathrm{Cp}), 87.5(\mathrm{C}), 115.2$ $\left(2 x \mathrm{CH}, \mathrm{C}_{6} \mathrm{H}_{4}\right), 128.1\left(2 \times \mathrm{CH}, \mathrm{C}_{6} \mathrm{H}_{4}\right), 128.4\left(\mathrm{CH}, \mathrm{C}_{6} \mathrm{H}_{4}\right), 129.9(\mathrm{C}), 130.3\left(\mathrm{CH}, \mathrm{C}_{6} \mathrm{H}_{4}\right), 132.7(\mathrm{C}), 133.0\left(\mathrm{CH}, \mathrm{C}_{6} \mathrm{H}_{4}\right)$, $137.5(\mathrm{C}), 138.8\left(2 \times \mathrm{CH}, \mathrm{C}_{6} \mathrm{H}_{4}\right), 157.5(\mathrm{C}), 172.1$ (2C, $\left.\mathrm{C}=\mathrm{O}\right), 185.9(\mathrm{C}, \mathrm{C}=\mathrm{O})$. HRMS calcd for $\mathrm{C}_{33} \mathrm{H}_{31} \mathrm{FeO}_{4}(\mathrm{M})^{+}$: 561.1603, found: 561.1597. 13-Indene. ${ }^{1} \mathrm{H}$ NMR $\left(400 \mathrm{MHz}\right.$, acetone- $\left.d_{6}\right): \delta=1.19\left(\mathrm{~m}, 1 \mathrm{H}, \mathrm{CH}_{2}\right), 1.41(\mathrm{~m}, 1 \mathrm{H}$, $\left.\mathrm{CH}_{2}\right), 1.85\left(\mathrm{~m}, J=6.6 \mathrm{~Hz}, 2 \mathrm{H} ; \mathrm{CH}_{2}\right), 1.96\left(\mathrm{~m}, 1 \mathrm{H}, \mathrm{CH}_{2}\right), 2.23\left(\mathrm{~m}, 1 \mathrm{H}, \mathrm{CH}_{2}\right), 2.55\left(\mathrm{~m}, 4 \mathrm{H}, 2 \mathrm{CH}_{2}\right), 3.62(\mathrm{~m}, 2 \mathrm{H}$, $\left.\mathrm{CH}_{2}\right), 3.81(\mathrm{~m}, 1 \mathrm{H}, \mathrm{CH}), 4.01(\mathrm{~s}, 5 \mathrm{H}, 5 \mathrm{xCH} ; \mathrm{Cp}), 4.04\left(\mathrm{~s}, 1 \mathrm{H}, \mathrm{C}_{5} \mathrm{H}_{4}\right), 4.15\left(\mathrm{~s}, 1 \mathrm{H}, \mathrm{C}_{5} \mathrm{H}_{4}\right), 4.22\left(\mathrm{~s}, 1 \mathrm{H}, \mathrm{C}_{5} \mathrm{H}_{4}\right), 4.43$ $\left(\mathrm{s}, 1 \mathrm{H}, \mathrm{C}_{5} \mathrm{H}_{4}\right), 6.68\left(\mathrm{~m}, 2 \mathrm{H}, \mathrm{C}_{6} \mathrm{H}_{3}\right), 6.97\left(\mathrm{~s}, 1 \mathrm{H}, \mathrm{C}_{6} \mathrm{H}_{3}\right), 7.02\left(\mathrm{~d}, J=8.5 \mathrm{~Hz}, 2 \mathrm{H}, \mathrm{C}_{6} \mathrm{H}_{4}\right), 7.22(\mathrm{~d}, J=8.4 \mathrm{~Hz} 2 \mathrm{H}$; $\left.\mathrm{C}_{6} \mathrm{H}_{4}\right), 8.16(\mathrm{~s}, 1 \mathrm{H}, \mathrm{OH}), 8.51(\mathrm{~s}, 1 \mathrm{H}, \mathrm{OH}) .{ }^{13} \mathrm{C} \mathrm{NMR}\left(100 \mathrm{MHz}\right.$, acetone- $\left.d_{6}\right): \delta=16.9\left(\mathrm{CH}_{2}\right), 23.0\left(\mathrm{CH}_{2}\right), 29.9$ $\left(\mathrm{CH}_{2}\right), 32.4\left(2 \mathrm{xCH}_{2}\right), 38.9\left(\mathrm{CH}_{2}\right), 50.1(\mathrm{CH}), 66.6\left(\mathrm{CH}, \mathrm{C}_{5} \mathrm{H}_{4}\right), 67.9\left(\mathrm{CH}, \mathrm{C}_{5} \mathrm{H}_{4}\right), 68.3\left(\mathrm{CH}, \mathrm{C}_{5} \mathrm{H}_{4}\right), 69.2(5 x \mathrm{CH}$, $\mathrm{Cp}), 69.5\left(\mathrm{CH}, \mathrm{C}_{5} \mathrm{H}_{4}\right), 81.2(\mathrm{C}), 110.6(\mathrm{CH}), 113.2(\mathrm{CH}), 115.4\left(2 \times \mathrm{CH}, \mathrm{C}_{6} \mathrm{H}_{4}\right), 119.6(\mathrm{CH}), 128.1(\mathrm{C}), 130.4$ (2xCH, $\left.\mathrm{C}_{6} \mathrm{H}_{4}\right), 139.4(\mathrm{C}), 139.9(\mathrm{C}), 148.6(\mathrm{C}), 155.3(\mathrm{C}), 156.8(\mathrm{C}), 172.2(2 \mathrm{C}, \mathrm{C}=\mathrm{O})$.

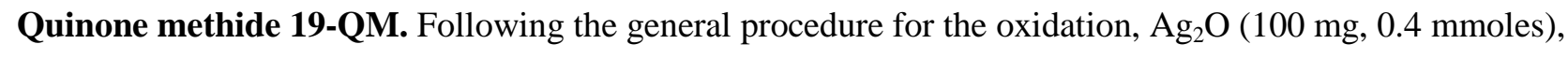
compound 19 (40 mg, 0.07 mmoles). Compound 19-QM was obtained as a dark red solid $38 \mathrm{mg}$, yield $95 \%$. ${ }^{1} \mathrm{H}$ NMR (400 MHz, acetone- $\left.d_{6}\right): \delta=1.66\left(\mathrm{dt}, J=14.9,7.6 \mathrm{~Hz}, 2 \mathrm{H}, \mathrm{CH}_{2}\right), 1.86\left(\mathrm{p}, J=6.5 \mathrm{~Hz}, 2 \mathrm{H}, \mathrm{CH}_{2}\right), 2.07(\mathrm{~m}, 2 \mathrm{H}$, $\left.\mathrm{CH}_{2}\right), 2.58\left(\mathrm{t}, J=6.5 \mathrm{~Hz}, 4 \mathrm{H}, 2 \mathrm{xCH}_{2}\right), 3.72\left(\mathrm{~m}, 2 \mathrm{H}, \mathrm{CH}_{2}\right), 4.00(\mathrm{~s}, 5 \mathrm{H}, 5 \mathrm{xCH} ; \mathrm{Cp}), 4.08\left(\mathrm{~m}, 1 \mathrm{H}, \mathrm{C}_{5} \mathrm{H}_{4}\right), 4.11(\mathrm{~s}$, 
$\left.1 \mathrm{H}, \mathrm{C}_{5} \mathrm{H}_{4}\right), 4.17\left(\mathrm{~s}, 1 \mathrm{H}, \mathrm{C}_{5} \mathrm{H}_{4}\right), 4.29\left(\mathrm{~s}, 1 \mathrm{H}, \mathrm{C}_{5} \mathrm{H}_{4}\right), 6.33-6.45(\mathrm{~m}, 3 \mathrm{H}, 2 \mathrm{xCH}+\mathrm{CH}), 7.43-7.58\left(\mathrm{~m}, 6 \mathrm{H}, \mathrm{C}_{6} \mathrm{H}_{4}\right)$, $7.64\left(\mathrm{dd}, J=10.1,2.7 \mathrm{~Hz}, 1 \mathrm{H}, \mathrm{C}_{6} \mathrm{H}_{4}\right) .{ }^{13} \mathrm{C}$ NMR (100 MHz, acetone- $\left.d_{6}\right): \delta=17.0\left(\mathrm{CH}_{2}\right), 27.4\left(\mathrm{CH}_{2}\right), 27.5\left(\mathrm{CH}_{2}\right)$, $32.4\left(2 \mathrm{xCH}_{2}\right), 38.4\left(\mathrm{CH}_{2}\right), 38.1\left(\mathrm{CH}_{2}\right), 66.7\left(\mathrm{CH}, \mathrm{C}_{5} \mathrm{H}_{4}\right), 67.1\left(\mathrm{CH}, \mathrm{C}_{5} \mathrm{H}_{4}\right), 68.0\left(2 \mathrm{xCH}, \mathrm{C}_{5} \mathrm{H}_{4}\right), 69.3(5 x \mathrm{CH}, \mathrm{Cp})$, $87.0(\mathrm{C}), 128.4\left(2 \times \mathrm{CH}, \mathrm{C}_{6} \mathrm{H}_{4}\right), 128.5(\mathrm{CH}), 128.9(\mathrm{CH}), 129.6(\mathrm{C}), 129.7(\mathrm{CH}), 129.8(\mathrm{CH}), 130.7\left(2 \times \mathrm{CH}_{2} \mathrm{C}_{6} \mathrm{H}_{4}\right)$, $137.4(\mathrm{CH}), 137.6(\mathrm{C}), 137.9(\mathrm{C}), 138.9(\mathrm{CH}), 156.7(\mathrm{C}), 172.2(2 \mathrm{C}, \mathrm{C}=\mathrm{O}), 186.0(\mathrm{C}, \mathrm{C}=\mathrm{O})$. HRMS calcd for $\mathrm{C}_{33} \mathrm{H}_{31} \mathrm{FeNO}_{3}(\mathrm{M})^{+}: 545.1653$, found: 545.1648 . 19-Indene. ${ }^{1} \mathrm{H}$ NMR (400 MHz, acetone- $\left.d_{6}\right): \delta=1.11(\mathrm{~m}, 1 \mathrm{H}$, $\left.\mathrm{CH}_{2}\right), 1.20\left(\mathrm{~m}, 1 \mathrm{H}, \mathrm{CH}_{2}\right), 1.80\left(\mathrm{~m}, 2 \mathrm{H}, \mathrm{CH}_{2}\right), 1.89\left(\mathrm{~m}, 1 \mathrm{H}, \mathrm{CH}_{2}\right), 2.22\left(\mathrm{~m}, 1 \mathrm{H}, \mathrm{CH}_{2}\right), 2.55\left(\mathrm{~m}, 4 \mathrm{H}, 2 \mathrm{CH}_{2}\right), 3.60$ (m, 2H, $\left.\mathrm{CH}_{2}\right), 3.86(\mathrm{~m}, 1 \mathrm{H}, \mathrm{CH}), 3.97\left(\mathrm{~s}, 1 \mathrm{H}, \mathrm{C}_{5} \mathrm{H}_{4}\right), 4.02(\mathrm{~s}, 5 \mathrm{H}, 5 \mathrm{xCH} ; \mathrm{Cp}), 4.11\left(\mathrm{~s}, 1 \mathrm{H}, \mathrm{C}_{5} \mathrm{H}_{4}\right), 4.23(\mathrm{~s}, 1 \mathrm{H}$, $\left.\mathrm{C}_{5} \mathrm{H}_{4}\right), 4.43\left(\mathrm{~s}, 1 \mathrm{H}, \mathrm{C}_{5} \mathrm{H}_{4}\right), 6.67\left(\mathrm{~m}, 2 \mathrm{H}, \mathrm{C}_{6} \mathrm{H}_{3}\right), 6.99\left(\mathrm{~s}, 1 \mathrm{H}, \mathrm{C}_{6} \mathrm{H}_{3}\right), 7.39-7.59\left(\mathrm{~m}, 5 \mathrm{H}, \mathrm{C}_{6} \mathrm{H}_{5}\right), 8.21(\mathrm{~s}, 1 \mathrm{H}, \mathrm{OH})$. ${ }^{13} \mathrm{C}$ NMR $\left(100 \mathrm{MHz}\right.$, acetone- $\left.d_{6}\right): \delta=16.9\left(\mathrm{CH}_{2}\right), 22.9\left(\mathrm{CH}_{2}\right), 29.9\left(\mathrm{CH}_{2}\right), 32.4\left(2 \mathrm{xCH}_{2}\right), 38.9\left(\mathrm{CH}_{2}\right), 50.2(\mathrm{CH})$, $66.7\left(\mathrm{CH}, \mathrm{C}_{5} \mathrm{H}_{4}\right), 68.0\left(\mathrm{CH}, \mathrm{C}_{5} \mathrm{H}_{4}\right), 68.3\left(\mathrm{CH}, \mathrm{C}_{5} \mathrm{H}_{4}\right), 68.5\left(\mathrm{CH}, \mathrm{C}_{5} \mathrm{H}_{4}\right), 69.3(5 x \mathrm{CH}, \mathrm{Cp}), 80.8(\mathrm{C}), 110.7(\mathrm{CH})$, $113.3(\mathrm{CH}), 119.5(\mathrm{CH}), 127.3(\mathrm{CH}), 128.5(\mathrm{CH}), 128.6\left(2 \mathrm{xCH}, \mathrm{C}_{6} \mathrm{H}_{5}\right), 129.5\left(2 \mathrm{xCH}_{1} \mathrm{C}_{6} \mathrm{H}_{5}\right), 137.3(\mathrm{C}), 137.5(\mathrm{C})$, 139.1 (C), 140.1 (C), $148.6(\mathrm{C}), 155.5$ (C), 172.2 (2C, C=O).

Quinone methide 14-QM. Following the general procedure for the oxidation, $\mathrm{Ag}_{2} \mathrm{O}(0.25 \mathrm{~g}, 1 \mathrm{mmoles})$, compound 14 (0.12 g, 0.2 mmoles). Compound 14-QM was obtained as a dark red solid $105 \mathrm{mg}$, yield 90\%. ${ }^{1} \mathrm{H}$ NMR (400 MHz, acetone- $\left.d_{6}\right): \delta=2.42\left(\mathrm{q}, J=6.5 \mathrm{~Hz}, 2 \mathrm{H}, \mathrm{CH}_{2}\right), 3.84\left(\mathrm{t}, J=6.3 \mathrm{~Hz}, 2 \mathrm{H}, \mathrm{CH}_{2}\right), 3.97(\mathrm{~s}, 5 \mathrm{H}$, $5 \times \mathrm{XH} ; \mathrm{Cp}), 4.05-4.20\left(\mathrm{~m}, 4 \mathrm{H}, \mathrm{C}_{5} \mathrm{H}_{4}\right), 6.02$ (br s, 1H, $\left.\mathrm{C}_{6} \mathrm{H}_{4}\right), 6.33\left(\right.$ br s, $\left.1 \mathrm{H}, \mathrm{C}_{6} \mathrm{H}_{4}\right), 6.43$ (t, J= 7.5 Hz, 1H; CH), 6.80 (br s, $2 \mathrm{H} ; \mathrm{C}_{6} \mathrm{H}_{4}$ ), 7.30 (br s, $2 \mathrm{H} ; \mathrm{C}_{6} \mathrm{H}_{4}$ ), 7.47 (br s, $2 \mathrm{H} ; \mathrm{C}_{6} \mathrm{H}_{4}$ ), 7.84 (s, $4 \mathrm{H} ; \mathrm{C}_{6} \mathrm{H}_{4}$ ), $9.02(\mathrm{~s}, 1 \mathrm{H}, \mathrm{OH}) .{ }^{13} \mathrm{C}$ NMR (100 MHz, acetone- $\left.d_{6}\right): \delta=29.7\left(\mathrm{CH}_{2}\right), 36.9\left(\mathrm{CH}_{2}\right), 68.1\left(2 \mathrm{xCH}, \mathrm{C}_{5} \mathrm{H}_{4}\right), 69.2\left(2 \mathrm{xCH}, \mathrm{C}_{5} \mathrm{H}_{4}\right), 69.3(5 x \mathrm{CH}$, $\mathrm{Cp}), 87.0(\mathrm{C}), 115.2\left(2 \mathrm{xCH}, \mathrm{C}_{6} \mathrm{H}_{4}\right), 122.9\left(2 \mathrm{xCH} ; \mathrm{C}_{6} \mathrm{H}_{4}\right), 123.0\left(\mathrm{CH} ; \mathrm{C}_{6} \mathrm{H}_{4}\right), 123.2(\mathrm{C}), 127.2\left(2 \mathrm{xCH} ; \mathrm{C}_{6} \mathrm{H}_{4}\right)$, 128.7 (C), 130.7 (C), $132.2\left(2 \times \mathrm{xH}_{6} \mathrm{C}_{6} \mathrm{H}_{4}\right), 132.3$ (C), 134.2 (2xCH; $\left.\mathrm{C}_{6} \mathrm{H}_{4}\right), 134.3(\mathrm{C}), 134.5$ (C), $139.6(2 \times \mathrm{CH}$; $\left.\mathrm{C}_{6} \mathrm{H}_{4}\right), 156.9(\mathrm{C}), 167.9(\mathrm{C}), 185.7$ (C). HRMS calcd for $\mathrm{C}_{35} \mathrm{H}_{27} \mathrm{FeNO}_{4}(\mathrm{M})^{+}$: 581.1290, found: 581.1284 . Quinone methide 20-QM. Following the general procedure for the oxidation, $\operatorname{Ag}_{2} \mathrm{O}(0.25 \mathrm{~g}, 1 \mathrm{mmoles})$, compound 20 ( $0.1 \mathrm{~g}, 0.2$ mmoles). Compound 20-QM was obtained as a dark red solid $95 \mathrm{mg}$, yield $84 \%$. ${ }^{1} \mathrm{H}$ NMR (400 MHz, acetone- $\left.d_{6}\right): \delta=2.47\left(\mathrm{q}, J=6.5 \mathrm{~Hz}, 2 \mathrm{H}, \mathrm{CH}_{2}\right), 3.86\left(\mathrm{t}, J=6.4 \mathrm{~Hz}, 2 \mathrm{H}, \mathrm{CH}_{2}\right), 3.94(\mathrm{~s}, 5 \mathrm{H}$, $5 \mathrm{xCH} ; \mathrm{Cp}), 4.09\left(\mathrm{~s}, 1 \mathrm{H}, \mathrm{C}_{5} \mathrm{H}_{4}\right), 4.11\left(\mathrm{~s}, 1 \mathrm{H}, \mathrm{C}_{5} \mathrm{H}_{4}\right), 4.15\left(\mathrm{~s}, 1 \mathrm{H}, \mathrm{C}_{5} \mathrm{H}_{4}\right), 4.24\left(\mathrm{~s}, 1 \mathrm{H}, \mathrm{C}_{5} \mathrm{H}_{4}\right), 6.06(\mathrm{dd}, J=10.0,2.0$ $\left.\mathrm{Hz}, 1 \mathrm{H}, \mathrm{C}_{6} \mathrm{H}_{4}\right), 6.32\left(\mathrm{dd}, J=10.1,2.0 \mathrm{~Hz}, 1 \mathrm{H}, \mathrm{C}_{6} \mathrm{H}_{4}\right), 6.44(\mathrm{t}, J=7.5 \mathrm{~Hz}, 1 \mathrm{H} ; \mathrm{CH}), 7.31-7.47\left(\mathrm{~m}, 6 \mathrm{H} ; \mathrm{C}_{6} \mathrm{H}_{4}\right)$, $7.50\left(\mathrm{dd}, J=10.0,2.7 \mathrm{~Hz}, 1 \mathrm{H}, \mathrm{C}_{6} \mathrm{H}_{4}\right), 7.85\left(\mathrm{~s}, 4 \mathrm{H} ; \mathrm{C}_{6} \mathrm{H}_{4}\right) .{ }^{13} \mathrm{C}$ NMR $\left(100 \mathrm{MHz}\right.$, acetone- $\left.d_{6}\right): \delta=29.7\left(\mathrm{CH}_{2}\right), 36.9$ $\left(\mathrm{CH}_{2}\right), 68.2\left(2 \mathrm{xCH}, \mathrm{C}_{5} \mathrm{H}_{4}\right), 69.2\left(2 \mathrm{xCH}, \mathrm{C}_{5} \mathrm{H}_{4}\right), 69.3(5 \mathrm{xCH}, \mathrm{Cp}), 86.6(\mathrm{C}), 123.0\left(2 \mathrm{xCH} ; \mathrm{C}_{6} \mathrm{H}_{4}\right), 127.0(\mathrm{CH}$; $\left.\mathrm{C}_{6} \mathrm{H}_{4}\right), 128.2(\mathrm{C}), 128.3\left(2 x \mathrm{xH} ; \mathrm{C}_{6} \mathrm{H}_{4}\right), 128.9\left(\mathrm{CH} ; \mathrm{C}_{6} \mathrm{H}_{4}\right), 129.7\left(\mathrm{CH} ; \mathrm{C}_{6} \mathrm{H}_{4}\right), 129.9(\mathrm{C}), 130.6\left(2 x C H ; \mathrm{C}_{6} \mathrm{H}_{4}\right)$, $131.0(\mathrm{C}), 132.3\left(\mathrm{CH} ; \mathrm{C}_{6} \mathrm{H}_{4}\right), 134.2\left(2 \times \mathrm{CH}_{6} \mathrm{C}_{6} \mathrm{H}_{4}\right), 134.3(\mathrm{C}), 137.2\left(\mathrm{CH} ; \mathrm{C}_{6} \mathrm{H}_{4}\right), 137.7(\mathrm{C}), 138.3\left(\mathrm{CH} ; \mathrm{C}_{6} \mathrm{H}_{4}\right)$, 139.4 (C), 155.9 (C), 185.7 (C). HRMS calcd for $\mathrm{C}_{35} \mathrm{H}_{27} \mathrm{FeNO}_{3}(\mathrm{M})^{+}$: 565.1340, found: 565.1335.

Quinone methide 15-QM. Following the general procedure for the oxidation, $\operatorname{Ag}_{2} \mathrm{O}(0.15 \mathrm{~g}, 6 \mathrm{mmoles})$, compound 15 (60 mg, 0.1 mmoles). Compound 15-QM was obtained as a dark red solid $55 \mathrm{mg}$, yield $92 \%$. ${ }^{1} \mathrm{H}$ NMR (400 MHz, acetone- $\left.d_{6}\right): \delta=1.83\left(\mathrm{~m}, J=6.7 \mathrm{~Hz}, 2 \mathrm{H}, \mathrm{CH}_{2}\right), 2.06\left(\mathrm{~m}, 2 \mathrm{H}, \mathrm{CH}_{2}\right), 3.65(\mathrm{t}, J=6.8 \mathrm{~Hz}, 2 \mathrm{H}$, $\mathrm{CH}_{2}$ ), 4.00 (s, 5H, 5xCH; Cp), $4.05-4.20\left(\mathrm{~m}, 4 \mathrm{H}, \mathrm{C}_{5} \mathrm{H}_{4}\right), 6.29$ (br s, $\left.2 \mathrm{H}, \mathrm{C}_{6} \mathrm{H}_{4}\right), 6.42$ (t, J = 7.5 Hz, 1H; CH), 
6.95 (br s, 2H, $\mathrm{C}_{6} \mathrm{H}_{4}$ ), 7.40 (br s, 4H; $\left.\mathrm{C}_{6} \mathrm{H}_{4}\right), 7.80$ (s, 4H; $\left.\mathrm{C}_{6} \mathrm{H}_{4}\right), 9.17$ (s, 1H, OH). ${ }^{13} \mathrm{C}$ NMR (100 MHz, acetone$\left.d_{6}\right): \delta=27.2\left(\mathrm{CH}_{2}\right), 28.0\left(\mathrm{CH}_{2}\right), 37.2\left(\mathrm{CH}_{2}\right), 66.9\left(2 \mathrm{xCH}, \mathrm{C}_{5} \mathrm{H}_{4}\right), 68.0\left(2 \mathrm{xCH}, \mathrm{C}_{5} \mathrm{H}_{4}\right), 69.3(5 \mathrm{xCH}, \mathrm{Cp}), 87.4(\mathrm{C})$, $115.5\left(2 x \mathrm{xH}, \mathrm{C}_{6} \mathrm{H}_{4}\right), 122.7(\mathrm{C}), 122.8\left(2 \mathrm{xCH} ; \mathrm{C}_{6} \mathrm{H}_{4}\right), 123.2(\mathrm{C}), 127.7\left(\mathrm{CH} ; \mathrm{C}_{6} \mathrm{H}_{4}\right), 128.7\left(\mathrm{CH} ; \mathrm{C}_{6} \mathrm{H}_{4}\right), 129.4$ $\left(2 \mathrm{xCH} ; \mathrm{C}_{6} \mathrm{H}_{4}\right), 132.1\left(2 \mathrm{xCH} ; \mathrm{C}_{6} \mathrm{H}_{4}\right), 133.0(\mathrm{C}), 134.0\left(2 \mathrm{xCH} ; \mathrm{C}_{6} \mathrm{H}_{4}\right), 134.5(\mathrm{C}), 137.6(\mathrm{C}), 138.1\left(\mathrm{CH}, \mathrm{C}_{6} \mathrm{H}_{4}\right)$, 139.0 (C), 157.5 (C), 167.8 (C), 185.9 (C). HRMS calcd for $\mathrm{C}_{36} \mathrm{H}_{29} \mathrm{FeNO}_{4}(\mathrm{M})^{+}:$595.1446, found: 595.1441. 15Indene. ${ }^{1} \mathrm{H}$ NMR $\left(400 \mathrm{MHz}\right.$, acetone- $\left.d_{6}\right): \delta=1.37\left(\mathrm{~m}, 1 \mathrm{H}, \mathrm{CH}_{2}\right), 1.55\left(\mathrm{~m}, 1 \mathrm{H}, \mathrm{CH}_{2}\right), 2.04\left(\mathrm{~m}, 1 \mathrm{H}, \mathrm{CH}_{2}\right), 2.30(\mathrm{~m}$, $\left.1 \mathrm{H}, \mathrm{CH}_{2}\right), 3.55\left(\mathrm{~m}, 2 \mathrm{H}, \mathrm{CH}_{2}\right), 3.84(\mathrm{~m}, 1 \mathrm{H}, \mathrm{CH}), 3.96(\mathrm{~s}, 5 \mathrm{H}, 5 \mathrm{xCH} ; \mathrm{Cp}), 4.00-4.10\left(\mathrm{~m}, 3 \mathrm{H}, \mathrm{C}_{5} \mathrm{H}_{4}\right), 4.33(\mathrm{~s}, 1 \mathrm{H}$, $\left.\mathrm{C}_{5} \mathrm{H}_{4}\right), 6.69\left(\mathrm{~m}, 2 \mathrm{H}, \mathrm{C}_{6} \mathrm{H}_{3}\right), 6.98\left(\mathrm{~s}, 1 \mathrm{H}, \mathrm{C}_{6} \mathrm{H}_{3}\right), 7.03\left(\mathrm{~d}, J=8.5 \mathrm{~Hz} 2 \mathrm{H} ; \mathrm{C}_{6} \mathrm{H}_{4}\right), 7.23\left(\mathrm{~d}, J=8.5 \mathrm{~Hz} 2 \mathrm{H} ; \mathrm{C}_{6} \mathrm{H}_{4}\right), 7.79$ (s, $\left.4 \mathrm{H}, \mathrm{C}_{6} \mathrm{H}_{4}\right), 8.23(\mathrm{~s}, 1 \mathrm{H}, \mathrm{OH}), 8.62(\mathrm{~s}, 1 \mathrm{H}, \mathrm{OH}) .{ }^{13} \mathrm{C}$ NMR $\left(100 \mathrm{MHz}\right.$, acetone- $\left.d_{6}\right): \delta=23.2\left(\mathrm{CH}_{2}\right), 29.2\left(\mathrm{CH}_{2}\right)$, $37.8\left(\mathrm{CH}_{2}\right), 49.9(\mathrm{CH}), 66.4\left(\mathrm{CH}, \mathrm{C}_{5} \mathrm{H}_{4}\right), 67.7\left(\mathrm{CH}, \mathrm{C}_{5} \mathrm{H}_{4}\right), 68.3\left(\mathrm{CH}, \mathrm{C}_{5} \mathrm{H}_{4}\right), 69.1(5 x \mathrm{xH}, \mathrm{Cp}), 69.9\left(\mathrm{CH}, \mathrm{C}_{5} \mathrm{H}_{4}\right)$, $80.9(\mathrm{C}), 110.6(\mathrm{CH}), 113.3(\mathrm{CH}), 115.4\left(2 \mathrm{xCH}, \mathrm{C}_{6} \mathrm{H}_{4}\right), 119.6(\mathrm{CH}), 122.8\left(2 \mathrm{xCH}_{1} \mathrm{C}_{6} \mathrm{H}_{4}\right), 123.2(\mathrm{CH}), 128.1(\mathrm{C})$, $130.6\left(2 x \mathrm{xH}, \mathrm{C}_{6} \mathrm{H}_{4}\right), 132.2(\mathrm{C}), 133.9$ (2xCH, $\left.\mathrm{C}_{6} \mathrm{H}_{4}\right), 134.5(\mathrm{C}), 138.4(\mathrm{C}), 139.6(\mathrm{C}), 148.4(\mathrm{C}), 155.5(\mathrm{C}), 156.8$ (C), 167.9 (2C, C=O).

Quinone methide 21-QM. Following the general procedure for the oxidation, $\mathrm{Ag}_{2} \mathrm{O}$ ( $\left.0.15 \mathrm{~g}, 6 \mathrm{mmoles}\right)$, compound 21 (0.071 g, 0.12 mmoles). Compound 21-QM was obtained as a dark red solid $65 \mathrm{mg}$, yield 92\%. ${ }^{1} \mathrm{H}$ NMR (400 MHz, acetone- $\left.d_{6}\right): \delta=1.86\left(\mathrm{p}, J=7.1 \mathrm{~Hz}, 2 \mathrm{H}, \mathrm{CH}_{2}\right), 2.13\left(\mathrm{~m}, 2 \mathrm{H}, \mathrm{CH}_{2}\right), 3.68\left(\mathrm{t}, J=6.9 \mathrm{~Hz}, 2 \mathrm{H}, \mathrm{CH}_{2}\right)$, 3.97 (s, 5H, 5xCH; Cp), $4.07\left(\mathrm{~s}, 1 \mathrm{H}, \mathrm{C}_{5} \mathrm{H}_{4}\right), 4.11\left(\mathrm{~s}, 1 \mathrm{H}, \mathrm{C}_{5} \mathrm{H}_{4}\right), 4.16\left(\mathrm{~s}, 1 \mathrm{H}, \mathrm{C}_{5} \mathrm{H}_{4}\right), 4.28\left(\mathrm{~s}, 1 \mathrm{H}, \mathrm{C}_{5} \mathrm{H}_{4}\right), 6.21(\mathrm{dd}, J$ $\left.=10.1,1.8 \mathrm{~Hz}, 1 \mathrm{H}, \mathrm{C}_{6} \mathrm{H}_{4}\right), 6.33\left(\mathrm{dd}, J=10.0,1.8 \mathrm{~Hz}, 1 \mathrm{H}, \mathrm{C}_{6} \mathrm{H}_{4}\right), 6.44(\mathrm{t}, J=7.5 \mathrm{~Hz}, 1 \mathrm{H} ; \mathrm{CH}), 7.35(\mathrm{dd}, J=10.1$, $\left.2.6 \mathrm{~Hz}, 1 \mathrm{H}, \mathrm{C}_{6} \mathrm{H}_{4}\right), 7.45-7.51\left(\mathrm{~m}, 5 \mathrm{H} ; \mathrm{C}_{6} \mathrm{H}_{5}\right), 7.61\left(\mathrm{dd}, J=10.0,2.6 \mathrm{~Hz}, 1 \mathrm{H}, \mathrm{C}_{6} \mathrm{H}_{4}\right), 7.81\left(\mathrm{~m}, 4 \mathrm{H} ; \mathrm{C}_{6} \mathrm{H}_{4}\right) .{ }^{13} \mathrm{C}$ NMR (100 MHz, acetone- $\left.d_{6}\right): \delta=27.4\left(\mathrm{CH}_{2}\right), 27.9\left(\mathrm{CH}_{2}\right), 37.2\left(\mathrm{CH}_{2}\right), 68.1\left(2 \mathrm{xCH}, \mathrm{C}_{5} \mathrm{H}_{4}\right), 69.1\left(\mathrm{CH}, \mathrm{C}_{5} \mathrm{H}_{4}\right), 69.3$ (5xCH, Cp), $69.5\left(\mathrm{CH}, \mathrm{C}_{5} \mathrm{H}_{4}\right), 87.0(\mathrm{C}), 122.8\left(2 \mathrm{xCH} ; \mathrm{C}_{6} \mathrm{H}_{4}\right), 128.2(\mathrm{C}), 128.4\left(2 \mathrm{xCH} \mathrm{C}_{6} \mathrm{H}_{4}\right), 128.5\left(\mathrm{CH} ; \mathrm{C}_{6} \mathrm{H}_{4}\right)$, $128.8\left(\mathrm{CH} ; \mathrm{C}_{6} \mathrm{H}_{4}\right), 129.2(\mathrm{C}), 129.7\left(\mathrm{CH} ; \mathrm{C}_{6} \mathrm{H}_{4}\right), 130.7\left(2 \times \mathrm{CH} ; \mathrm{C}_{6} \mathrm{H}_{4}\right), 131.1(\mathrm{C}), 132.1(\mathrm{C}), 134.0\left(2 \times C H ; \mathrm{C}_{6} \mathrm{H}_{4}\right)$, $137.1\left(\mathrm{CH} ; \mathrm{C}_{6} \mathrm{H}_{4}\right), 137.9\left(\mathrm{CH} ; \mathrm{C}_{6} \mathrm{H}_{4}\right), 138.6\left(\mathrm{CH} ; \mathrm{C}_{6} \mathrm{H}_{4}\right), 156.4(\mathrm{C}), 185.8(\mathrm{C})$. HRMS calcd for $\mathrm{C}_{36} \mathrm{H}_{29} \mathrm{FeNO}_{3}$ $(\mathrm{M})^{+}:$579.1497, found: 579.1491 .

Quinone methide 11-QM. Following the general procedure for the oxidation, $\operatorname{Ag}_{2} \mathrm{O}$ ( $\left.0.1 \mathrm{~g}, 4.3 \mathrm{mmoles}\right)$, compound 11 (0.045 g, 0.08 mmoles). Compound 11-QM was obtained as a dark red solid $41 \mathrm{mg}$, yield 91\%. ${ }^{1} \mathrm{H}$ NMR $\left(400 \mathrm{MHz}\right.$, acetone- $\left.d_{6}\right): \delta=1.33\left(\mathrm{~m}, 2 \mathrm{H}, \mathrm{CH}_{2}\right), 1.41\left(\mathrm{~m}, 2 \mathrm{H}, \mathrm{CH}_{2}\right), 1.98\left(\mathrm{~m}, 2 \mathrm{H}, \mathrm{CH}_{2}\right), 2.55\left(\mathrm{~s}, 4 \mathrm{H}, 2 \mathrm{CH}_{2}\right)$, $3.28\left(\mathrm{t}, J=7.0 \mathrm{~Hz}, 2 \mathrm{H}, \mathrm{CH}_{2}\right), 3.90(\mathrm{~s}, 5 \mathrm{H}, 5 \mathrm{xCH} ; \mathrm{Cp}), 4.01\left(\mathrm{~m}, 2 \mathrm{H}, \mathrm{C}_{5} \mathrm{H}_{4}\right), 4.07\left(\mathrm{~s}, 1 \mathrm{H}, \mathrm{C}_{5} \mathrm{H}_{4}\right), 4.17\left(\mathrm{~s}, 1 \mathrm{H}, \mathrm{C}_{5} \mathrm{H}_{4}\right)$, $6.25(\mathrm{~m}, J=7.5 \mathrm{~Hz}, 3 \mathrm{H}, 2 \mathrm{xCH}+\mathrm{CH}), 6.97$ (br s, $\left.2 \mathrm{H} ; \mathrm{C}_{6} \mathrm{H}_{4}\right), 7.32\left(\mathrm{br} \mathrm{s}, 2 \mathrm{H} ; \mathrm{C}_{6} \mathrm{H}_{4}\right), 7.50$ (br s, 2H; $\left.\mathrm{C}_{6} \mathrm{H}_{4}\right), 9.01$ (s, $1 \mathrm{H}, \mathrm{OH}) .{ }^{13} \mathrm{C}$ NMR $\left(100 \mathrm{MHz}\right.$, acetone- $\left.d_{6}\right): \delta=26.2\left(\mathrm{CH}_{2}\right), 26.6\left(\mathrm{CH}_{2}\right), 27.3\left(\mathrm{CH}_{2}\right), 27.9\left(2 \mathrm{xCH}_{2}\right), 37.8\left(\mathrm{CH}_{2}\right)$, $66.7\left(\mathrm{CH}, \mathrm{C}_{5} \mathrm{H}_{4}\right), 66.9\left(\mathrm{CH}, \mathrm{C}_{5} \mathrm{H}_{4}\right), 68.0\left(2 \mathrm{xCH}, \mathrm{C}_{5} \mathrm{H}_{4}\right), 69.2(5 \mathrm{xCH}, \mathrm{Cp}), 87.5(\mathrm{C}), 115.5\left(2 \times \mathrm{CH}, \mathrm{C}_{6} \mathrm{H}_{4}\right), 127.7$ $(\mathrm{CH}), 128.4(\mathrm{C}), 128.6\left(\mathrm{CH} ; \mathrm{C}_{6} \mathrm{H}_{4}\right), 130.3\left(2 \times \mathrm{CH}_{6} \mathrm{C}_{6} \mathrm{H}_{4}\right), 132.2(\mathrm{C}), 133.1(\mathrm{CH}), 137.7\left(2 \mathrm{xCH} ; \mathrm{C}_{6} \mathrm{H}_{4}\right), 137.8(\mathrm{C})$, 139.3 (C), 157.8 (C), 177.1 (2C, C=O), 185.9 (C, C=O). HRMS calcd for $\mathrm{C}_{33} \mathrm{H}_{31} \mathrm{FeO}_{4}(\mathrm{M})^{+}:$: 561.1603, found: 561.1597. 11-Indene. ${ }^{1} \mathrm{H}$ NMR $\left(400 \mathrm{MHz}\right.$, acetone- $\left.d_{6}\right): \delta=1.04\left(\mathrm{~m}, 1 \mathrm{H}, \mathrm{CH}_{2}\right), 1.29\left(\mathrm{~m}, 1 \mathrm{H}, \mathrm{CH}_{2}\right), 1.48(\mathrm{~m}, 2 \mathrm{H}$, $\left.\mathrm{CH}_{2}\right), 1.89\left(\mathrm{~m}, 1 \mathrm{H}, \mathrm{CH}_{2}\right), 2.26\left(\mathrm{~m}, 1 \mathrm{H}, \mathrm{CH}_{2}\right), 2.60\left(\mathrm{~s}, 4 \mathrm{H}, 2 \mathrm{xCH}_{2}\right), 3.34\left(\mathrm{~m}, 2 \mathrm{H}, \mathrm{CH}_{2}\right), 3.80(\mathrm{~m}, 1 \mathrm{H}, \mathrm{CH}), 4.01(\mathrm{~s}$, $5 \mathrm{H}, 5 \mathrm{xCH} ; \mathrm{Cp}), 4.07\left(\mathrm{~s}, 1 \mathrm{H}, \mathrm{C}_{5} \mathrm{H}_{4}\right), 4.12\left(\mathrm{~s}, 1 \mathrm{H}, \mathrm{C}_{5} \mathrm{H}_{4}\right), 4.20\left(\mathrm{~s}, 1 \mathrm{H}, \mathrm{C}_{5} \mathrm{H}_{4}\right), 4.42\left(\mathrm{~s}, 1 \mathrm{H}, \mathrm{C}_{5} \mathrm{H}_{4}\right), 6.69(\mathrm{~m}, 2 \mathrm{H}$, 
$\left.\mathrm{C}_{6} \mathrm{H}_{3}\right), 6.97\left(\mathrm{~s}, 1 \mathrm{H}, \mathrm{C}_{6} \mathrm{H}_{3}\right), 7.04\left(\mathrm{~d}, J=8.5 \mathrm{~Hz} 2 \mathrm{H} ; \mathrm{C}_{6} \mathrm{H}_{4}\right), 7.23\left(\mathrm{~d}, J=8.5 \mathrm{~Hz} 2 \mathrm{H} ; \mathrm{C}_{6} \mathrm{H}_{4}\right), 8.18(\mathrm{~s}, 1 \mathrm{H}, \mathrm{OH}), 8.55(\mathrm{~s}$, $1 \mathrm{H}, \mathrm{OH}) .{ }^{13} \mathrm{C}$ NMR $\left(100 \mathrm{MHz}\right.$, acetone- $\left.d_{6}\right): \delta=21.6\left(\mathrm{CH}_{2}\right), 27.6\left(\mathrm{CH}_{2}\right), 27.8\left(2 \mathrm{xCH}_{2}\right), 32.5\left(\mathrm{CH}_{2}\right), 37.9\left(\mathrm{CH}_{2}\right)$, $50.3(\mathrm{CH}), 66.5\left(\mathrm{CH}, \mathrm{C}_{5} \mathrm{H}_{4}\right), 67.9\left(\mathrm{CH}, \mathrm{C}_{5} \mathrm{H}_{4}\right), 68.2\left(\mathrm{CH}, \mathrm{C}_{5} \mathrm{H}_{4}\right), 68.4\left(\mathrm{CH}, \mathrm{C}_{5} \mathrm{H}_{4}\right), 69.1(5 \mathrm{xCH}, \mathrm{Cp}), 81.1(\mathrm{C})$, $110.8(\mathrm{CH}), 113.2(\mathrm{CH}), 115.4\left(2 \mathrm{xCH}, \mathrm{C}_{6} \mathrm{H}_{4}\right), 119.5(\mathrm{CH}), 128.2(\mathrm{C}), 130.6\left(2 \mathrm{xCH}, \mathrm{C}_{6} \mathrm{H}_{4}\right), 139.3(\mathrm{C}), 139.5(\mathrm{C})$, $148.8(\mathrm{C}), 155.3(\mathrm{C}), 156.8(\mathrm{C}), 177.1(2 \mathrm{C}, \mathrm{C}=\mathrm{O})$.

Variable-Temperature and decoupling NMR experimental procedures. Variable temperature NMR experiments were performed from $27{ }^{\circ} \mathrm{C}$ to $-70{ }^{\circ} \mathrm{C}$ on a Bruker Avance $400 \mathrm{MHz}$ NMR spectrometer. Data acquisition and processing were performed with TOPSPIN 2.1 program (Bruker). The desired temperature was set on the variable temperature unit and the sample was equilibrated for 10 15 minutes at each set temperature. The ${ }^{1} \mathrm{H}-\mathrm{NMR}$ spectra were recorded using a pulse sequence of proton with a spectral width of $7184 \mathrm{~Hz}$, an acquisition time of $4,5 \mathrm{~s}$ and a relaxation delay of $1 \mathrm{~s}$.

The homodecoupling spectra were recorded with the same parameters as that of 1H-NMR experiments (pulse program name: zghd). The decoupling power is optimized between $20 \mathrm{~dB}$ to $50 \mathrm{~dB}$. Finally, a suitable decoupling power at $35 \mathrm{~dB}$ was chosed for the experiments as it allows to irradiate the signal of interest without attenuating those nearby. The calculation of energy barrier to this process could be evaluated from peak coalescence according to the following equation: $\Delta \mathrm{G}^{\ddagger} /\left(R \cdot T_{\mathrm{c}}\right)=\log _{\mathrm{e}}(\sqrt{ } 2 \cdot R / \pi \cdot N \cdot h)+\log _{\mathrm{e}}\left(T_{\mathrm{c}} / \delta_{\mathrm{v}}\right)$.

$X$-ray crystal structure determinations for 14, Z-16, Z-20, 17, E-4-QM and E-12-QM. A single crystal of each compound was selected, mounted onto a cryoloop and transferred into a cold nitrogen gas stream. Intensity data were collected with a Bruker Kappa-APEXII diffractometer using graphite-monochromated Mo-K $\alpha$ radiation $(\lambda=$ $0.71073 \AA$ ). Data collection was performed with the Bruker APEXII suite. Unit-cell parameters determination, integration and data reduction were carried out with SAINT program. SADABS was used for scaling and absorption corrections. The structures were solved with SHELXT-2014 ${ }^{2}$ and refined by full-matrix least-squares methods with SHELXL-2014 ${ }^{3}$ using the WinGX suite ${ }^{4}$ or Olex 2 software package ${ }^{5}$. All non-hydrogen atoms were refined anisotropically. The structures were deposited at the Cambridge Crystallographic Data Centre with numbers CCDC 1860092 to 1860097 and can be obtained free of charge via www.ccdc.cam.ac.uk.

Kinetic experiments of selected QMs monitored by $\boldsymbol{U V}$-Vis spectroscopy. The disappearance of QMs $(25 \mu \mathrm{M})$ in $50 \mathrm{mM}$ phosphate buffer $\left(1 \mathrm{~mL}, \mathrm{pH} 7.4,6.0\right.$ or $\left.5.0,37^{\circ} \mathrm{C}\right)$ was followed by monitoring the decrease in UV absorbance at $430 \mathrm{~nm}(1 \mathrm{~min} / \mathrm{scan})$ using a Cary 50 Scan UV/VIS spectrophotometer. Pseudo first rate constants were determined in duplicate for at least three half-lives.

Measurements of pKa values of selected QMs. By using UV-Vis spectroscopy, one can plot log[In-/HIn] vs. pH and then apply for using Beer-Lambert Law in conjunction with the Henderson-Hasselbalch to calculate for the approximate $\mathrm{pKa}$ value of QMs.

$$
\log [\mathrm{In}-/ \mathrm{HIn}]=\mathrm{pH}-\mathrm{pKa}
$$




\section{Computational Methods}

All electronic structure calculations were performed with using the Gaussian program. ${ }^{6}$ Structural optimizations were carried out at Density Functional Theory (DFT) level using the B3LYP exchange correlation functional, including an empirical dispersion correction (D3-BJ) ${ }^{9}$ and treating solvent (here acetone) implicitly using the Polarizable Continuum Model (PCM) ${ }^{10}$ as implemented in Gaussian. Diffuse-augmented polarization valencedouble- $\zeta(6-31+\mathrm{G}(\mathrm{d}))^{11}$ basis set with one set of $d$ polarization functions, ${ }^{12,13}$ a set of $s$ and $p$ diffuse functions for all atoms but the hydrogens atoms, and the double- $\zeta$ quality LANL2DZ ${ }^{14}$ basis set with its associated effective core potential $^{15}$ (including 10 core electrons) for the iron atom were used. This basis set will be denoted hereafter as 'BS1'. Subsequently, vibrational frequency calculations were performed at the same level of theory in order to confirm that all the structures found were minima. In order to compare the relative stabilities of the different conformers, single point energy calculations were performed on optimized structures at using the Grimme's double-hybrid functional B2PLYP ${ }^{16}$ combined with the dispersion correction scheme (D3-BJ) ${ }^{10}$ and using the non-empirical double hybrid functional PBE0-DH ${ }^{17}$.

For all systems, two different conformers, presenting $1 p-\pi$ interaction or not, were considered.

The UV-Vis absorption spectra of the conformer with the lp- $\pi$ interaction of the 4-QM, 12-QM and 14-QM compounds (in the phenol protonated and deprotonated form, as shown in Figure 5 of the main text) were computed at Time Dependent-DFT level in acetone using the long-range corrected CAM-B3LYP ${ }^{18}$ functional together with the BS1 basis set. The natural transition orbitals ${ }^{19}$ (NTO) involved in the most intense low energy transition were computed in order to analyze the nature of the electronic transitions.

\section{Biological studies}

Culture cells. Stock solutions $(10 \mathrm{mM})$ of the compounds to be tested were prepared in DMSO and were kept at $20^{\circ} \mathrm{C}$ in the dark. Serial dilutions in Dulbecco's modified eagle medium (DMEM) without phenol red/Glutamax I were prepared just prior to use. DMEM without phenol red, Glutamax I and fetal bovine serum were purchased from Gibco; MDA-MB-231 cells were obtained from ATCC (Manassas, VA, USA). Cells were maintained in a monolayer culture in DMEM with phenol red/Glutamax I supplemented with $9 \%$ fetal bovine serum at $37^{\circ} \mathrm{C}$ in a $5 \% \mathrm{CO}_{2}$ /air-humidified incubator. For proliferation assays, MDA-MB-231 cells were plated in $1 \mathrm{~mL}$ of DMEM without phenol red, supplemented with $9 \%$ decomplemented and hormone-depleted fetal bovine serum, $1 \%$ kanamycin, 1\% Glutamax I and incubated. The following day (D0), $1 \mathrm{~mL}$ of the same medium containing the compounds to be tested was added to the plates. After 3 days (D3) the incubation medium was removed and $2 \mathrm{~mL}$ of the fresh medium containing the compounds was added. At different days (D4, D5), the protein content of each well was quantified by methylene blue staining as follows: cell monolayers were fixed for $1 \mathrm{~h}$ at room temperature with methylene blue (1mg mL-1 in 50:50 water/MeOH mixture), then washed with water. After addition of $\mathrm{HCl}(0.1 \mathrm{M}, 2 \mathrm{~mL})$, the plate was incubated for $1 \mathrm{~h}$ at $37{ }^{\circ} \mathrm{C}$ and then the absorbance of each well (4 wells for each concentration) was measured at $655 \mathrm{~nm}$ with a Biorad spectrophotometer. The results are 
expressed as the percentage of proteins versus the control. Two independent experiments, run in quadruplicate, were performed.

NCI/DTP cytotoxicity tests. The protocol for the determination of cytotoxicity on the 60 cell line panel can be found at https://dtp.cancer.gov/discovery development/nci-60/methodology.htm; The DTP homepage can be accessed at http://dtp.cancer.gov/. 


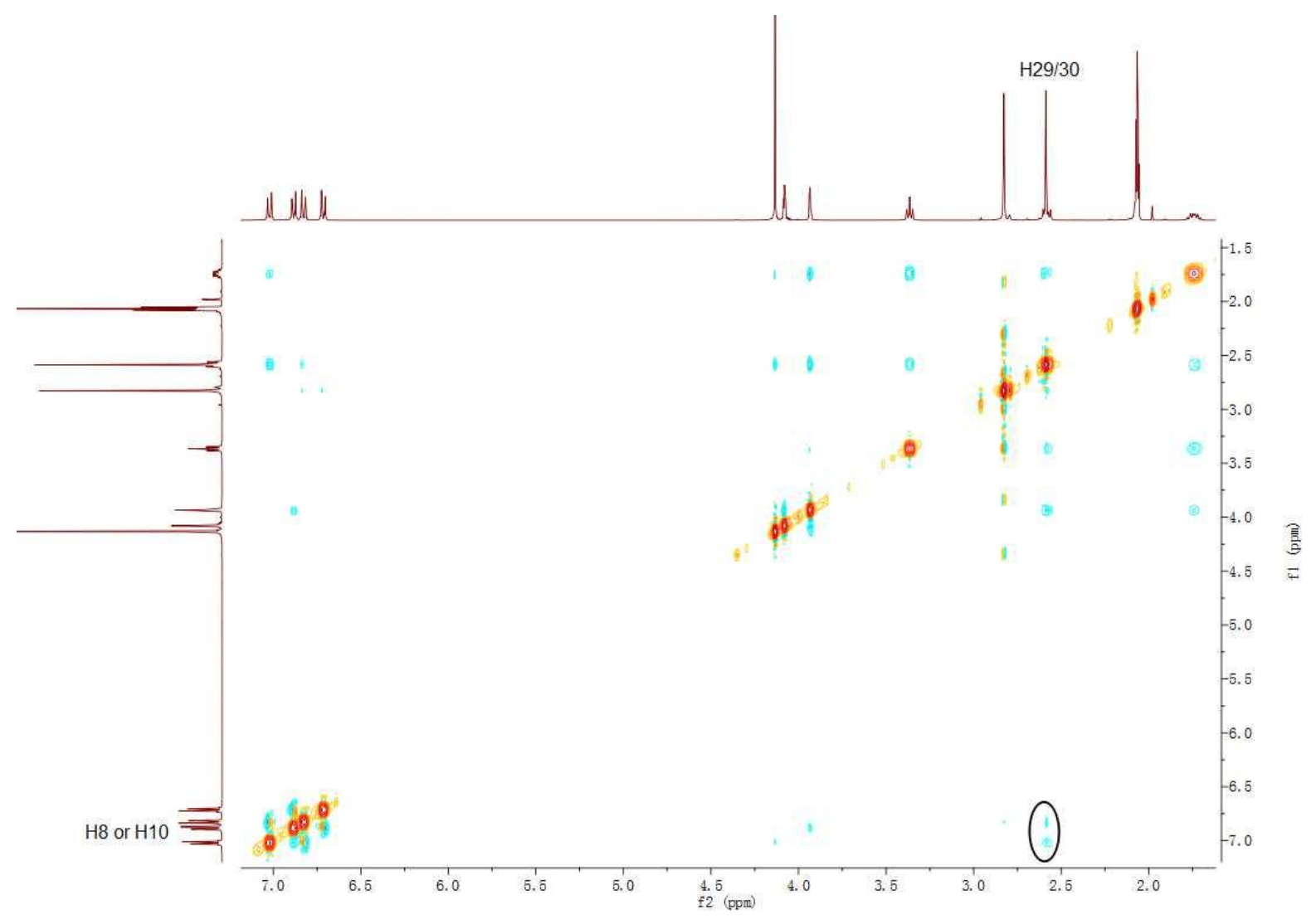

Fig. SI1. 2D-NOESY spectrum of $\mathbf{4}$ in acetone- $d_{6}$.

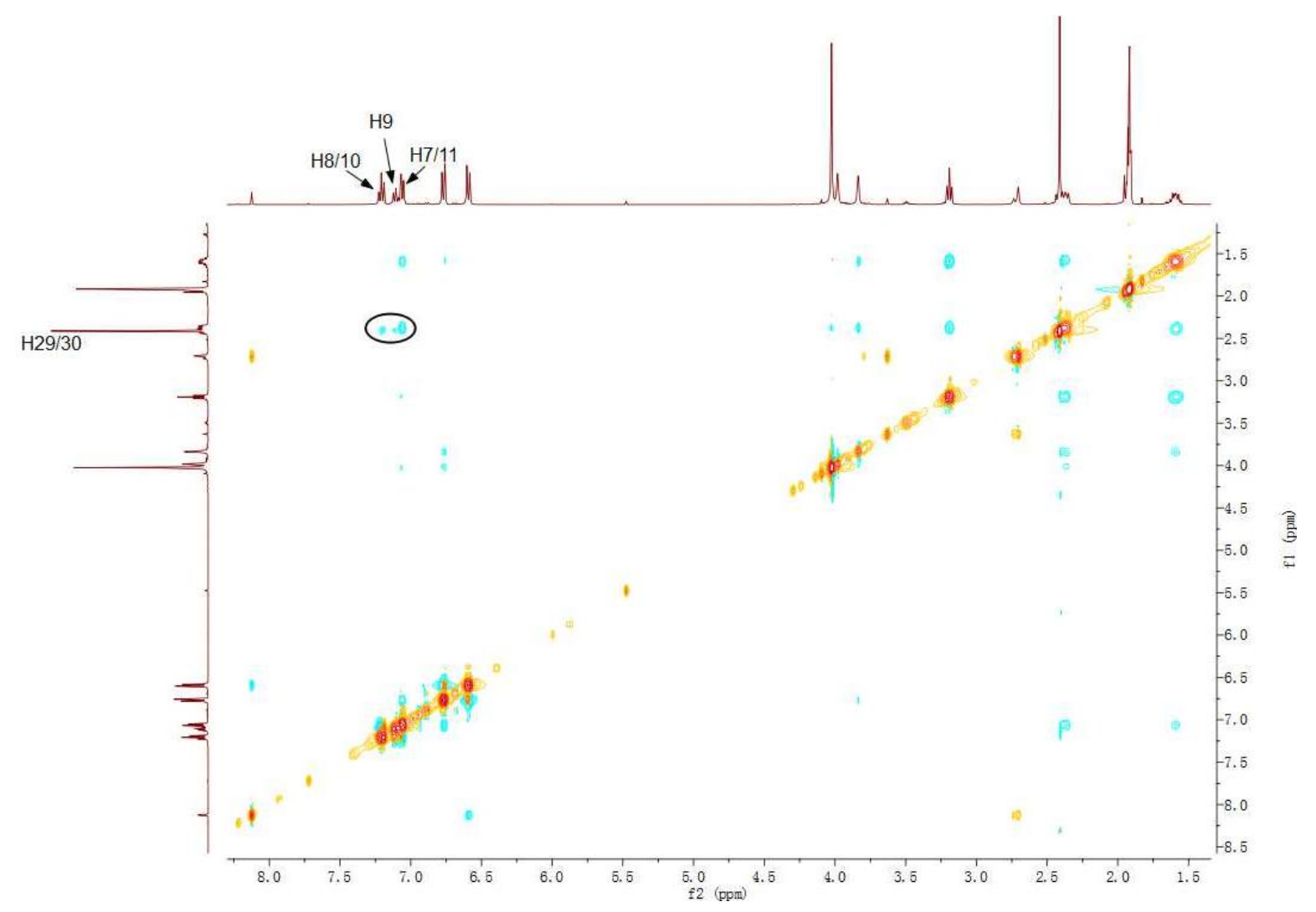


Fig. SI2. 2D-NOESY spectrum of Z-16 in acetone- $d_{6}$.

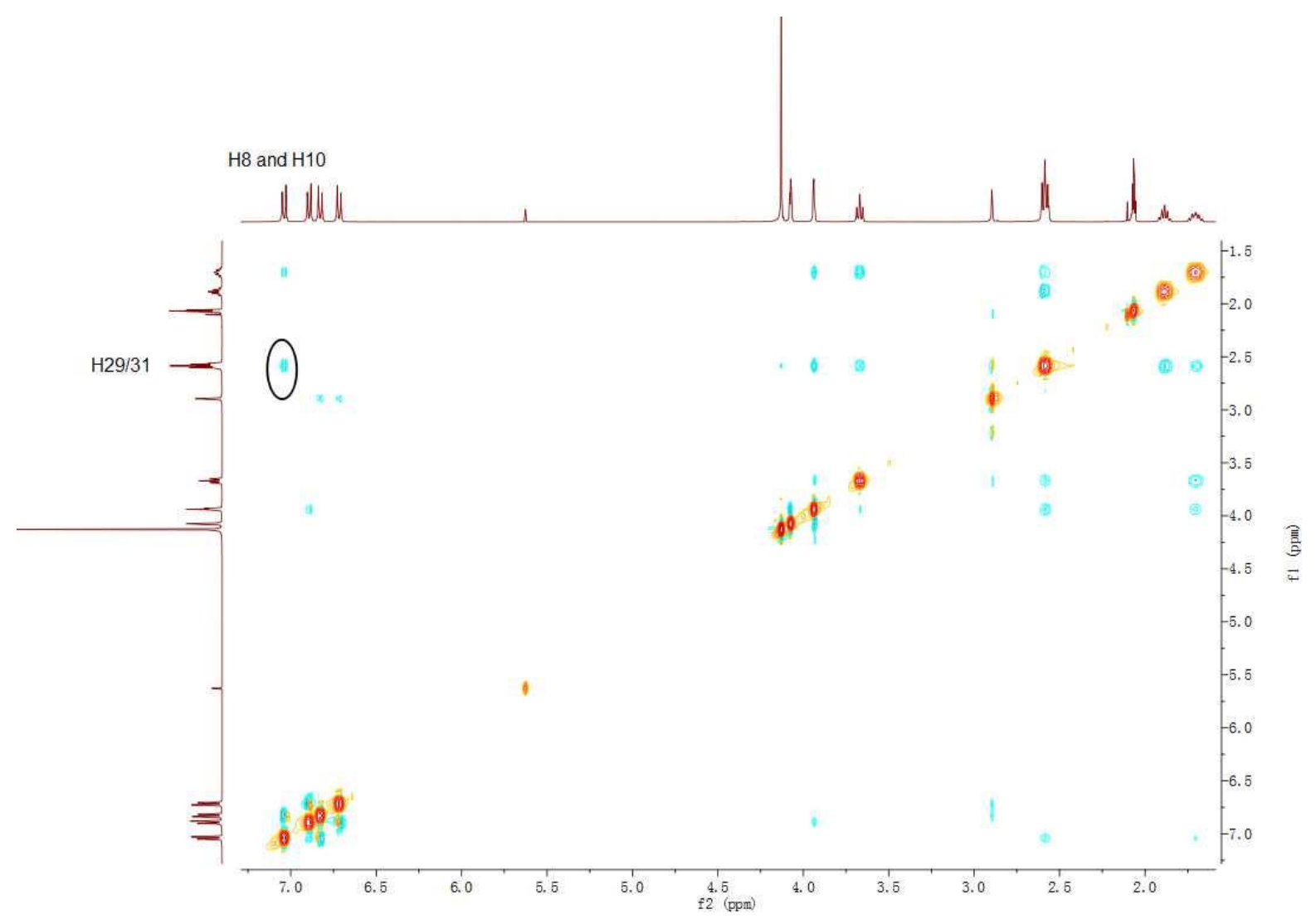

Fig. SI3. 2D-NOESY spectrum of $\mathbf{1 2}$ in acetone- $d_{6}$. 


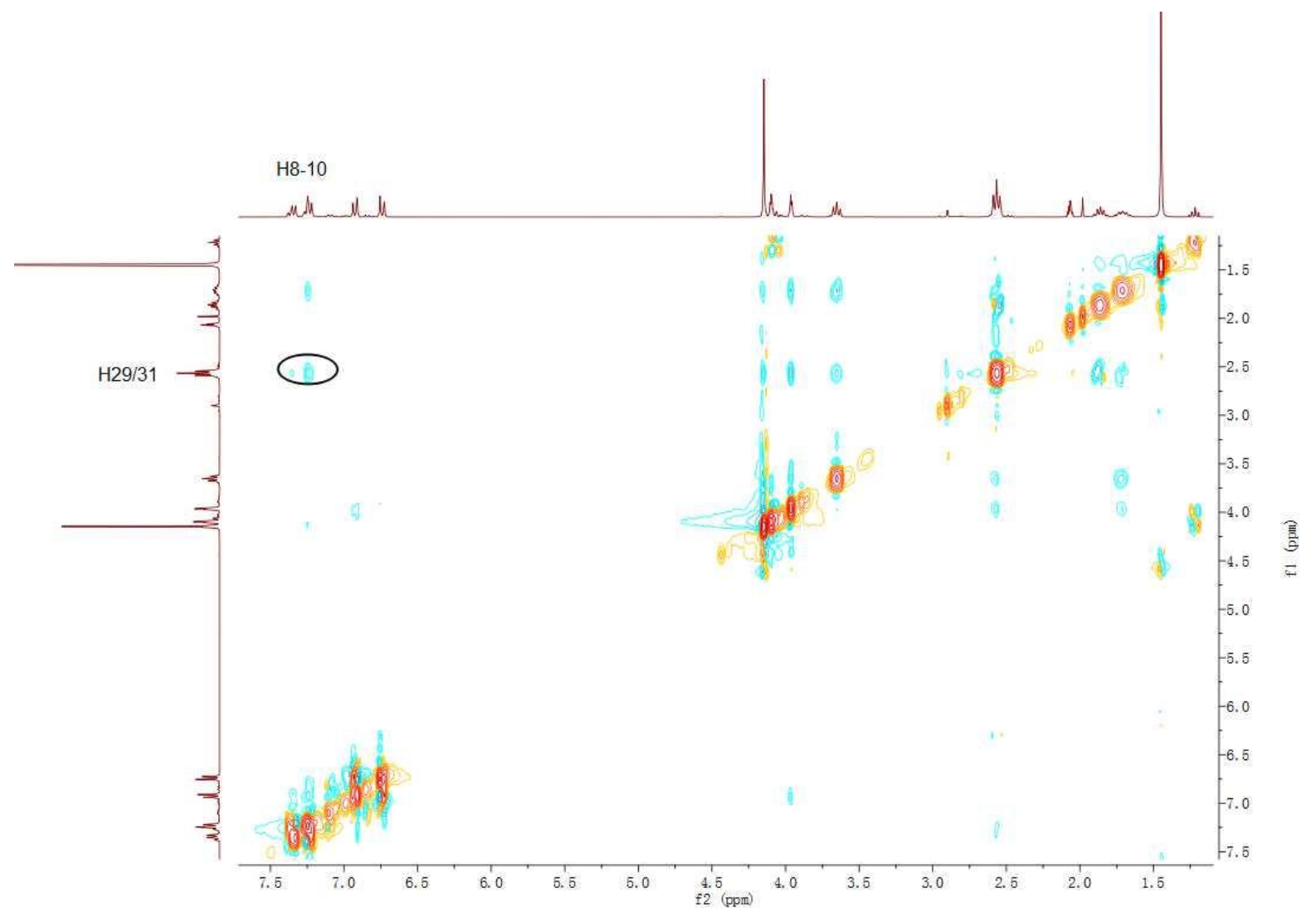

Fig. SI4. 2D-NOESY spectrum of Z-18 in acetone- $d_{6}$.

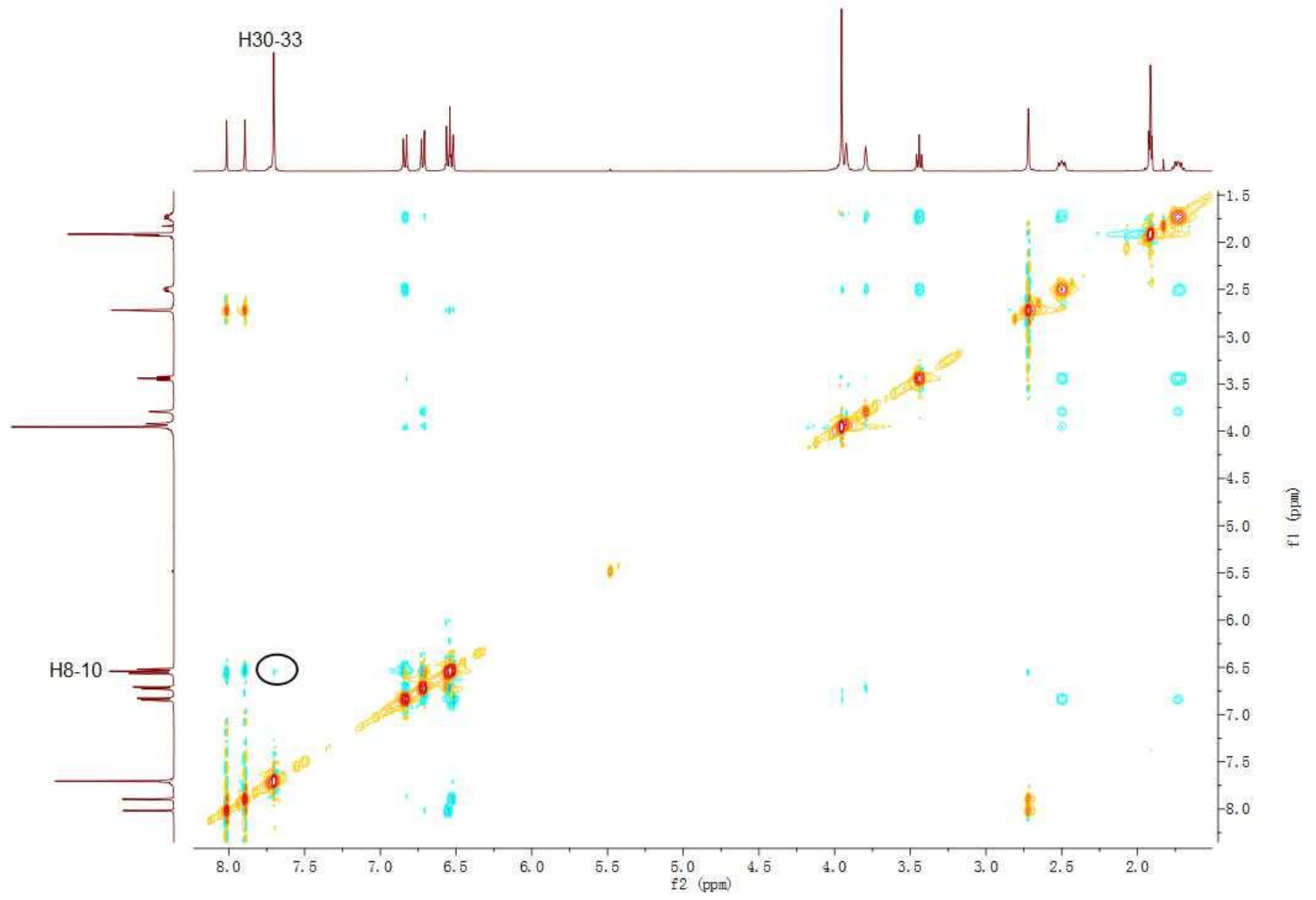


Fig. SI5. 2D-NOESY spectrum of $\mathbf{1 4}$ in acetone- $d_{6}$.

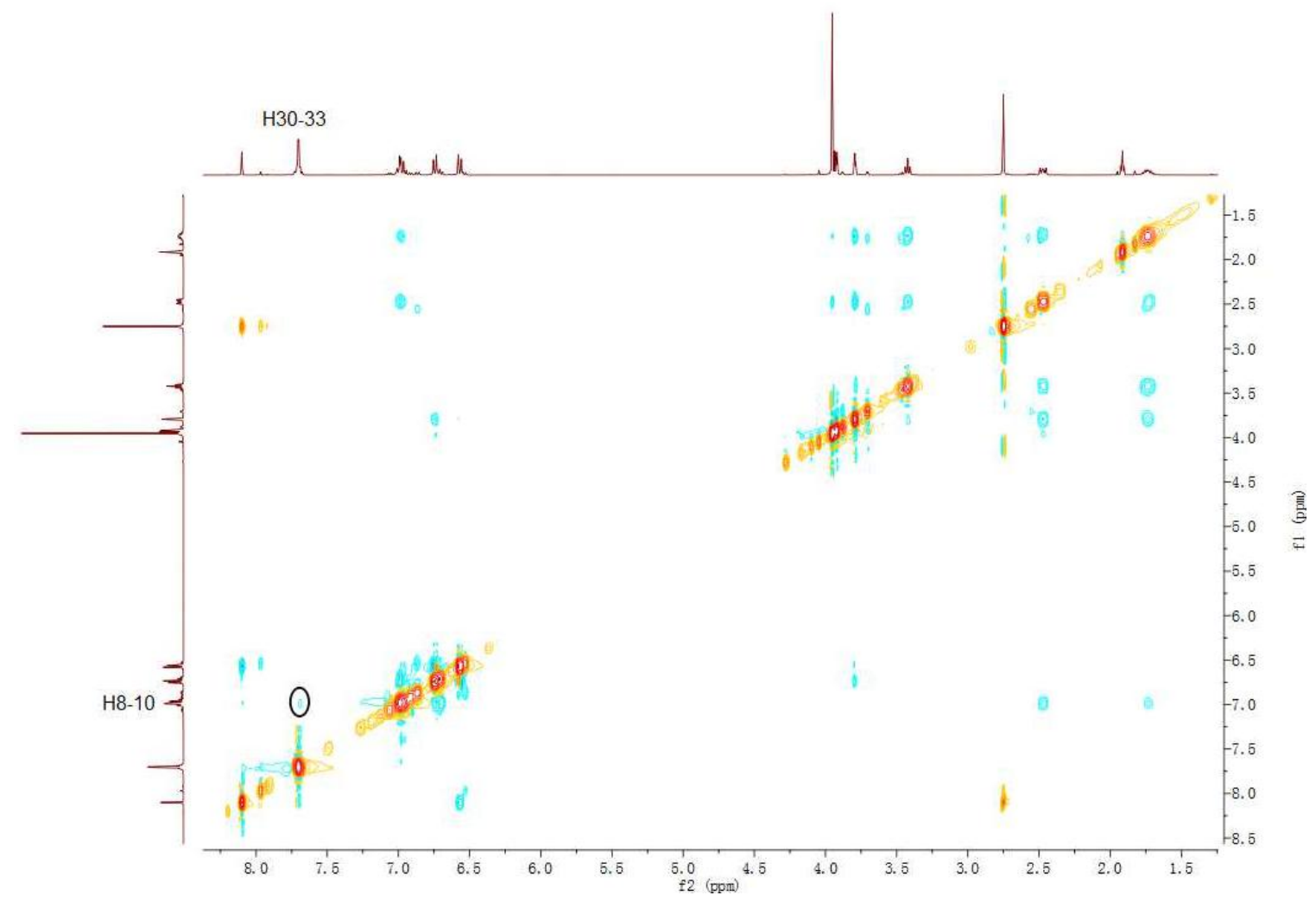

Fig. SI6. 2D-NOESY spectrum of Z-20 in acetone- $d_{6}$.
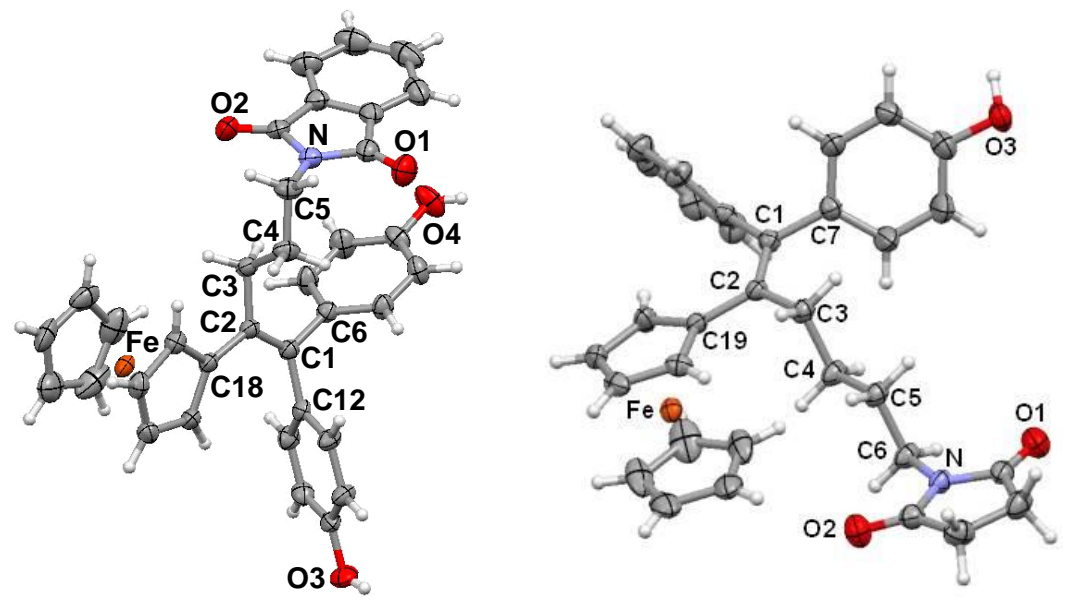

Figure SI7. Molecular structure of $\mathbf{1 4}$ and 17, thermal ellipsoids are shown at $50 \%$. 


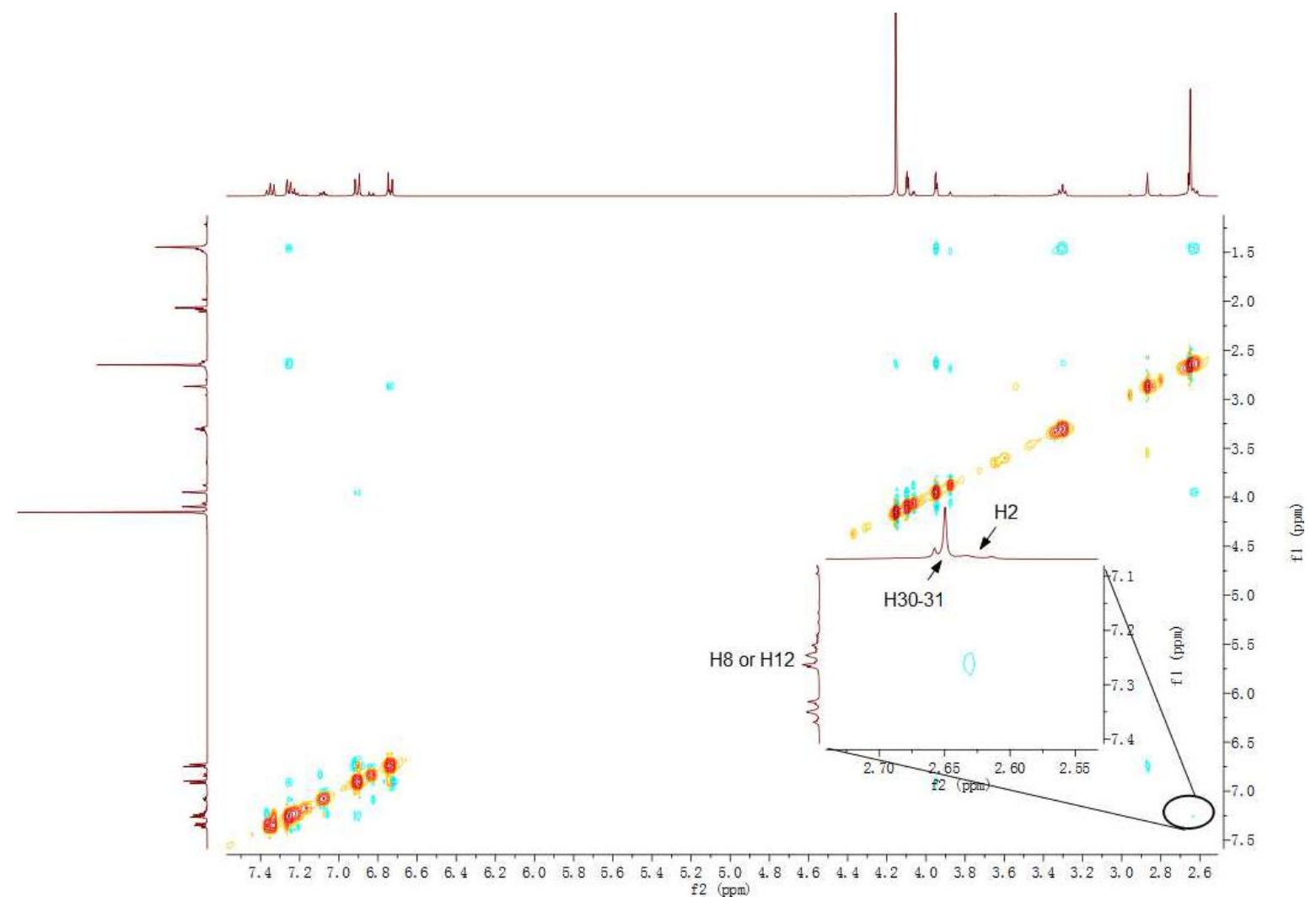

Fig. SI8. 2D-NOESY spectrum of Z-17 in acetone- $d_{6}$. 


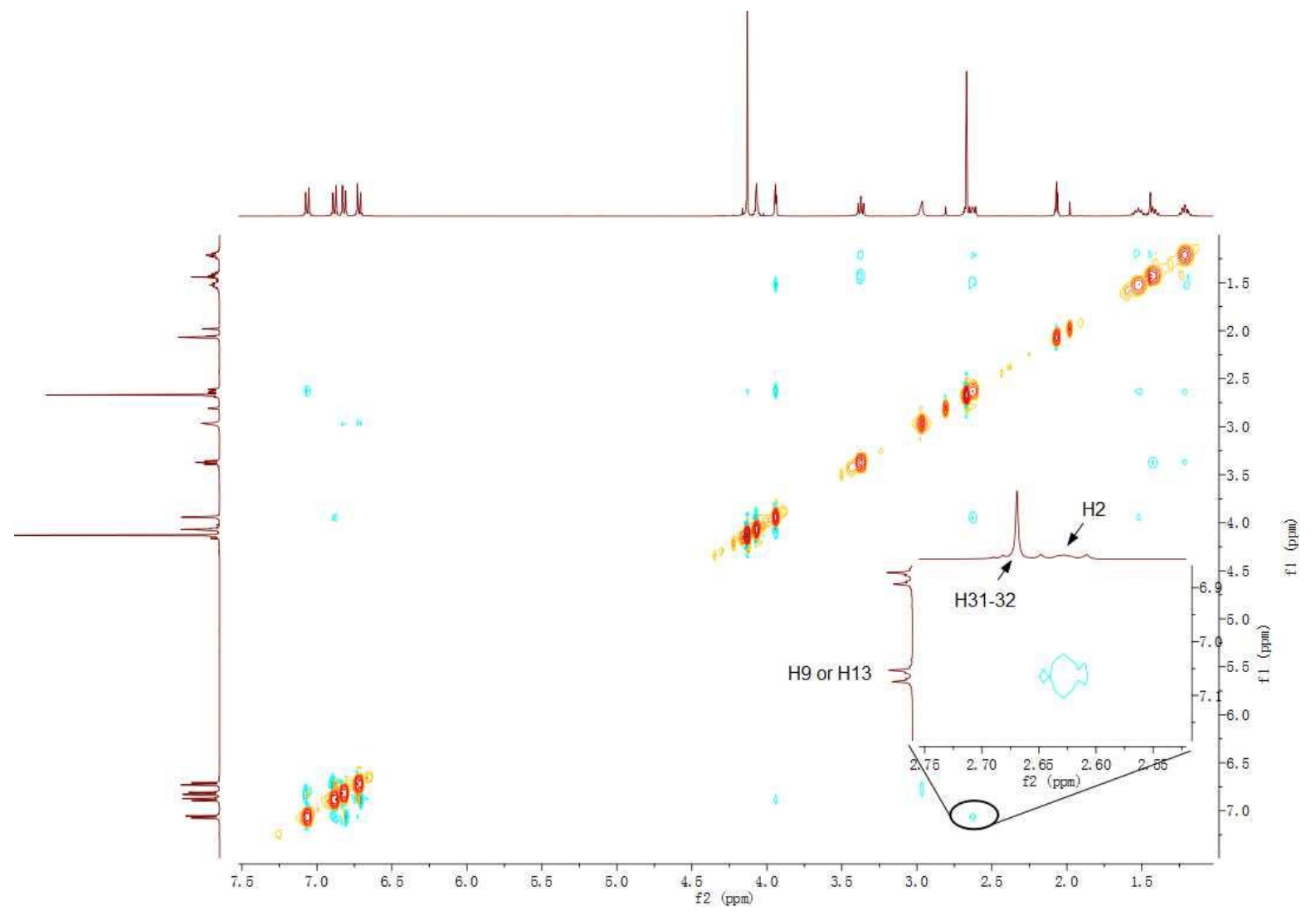

Fig. SI9. 2D-NOESY spectrum of $\mathbf{1 1}$ in acetone- $d_{6}$.

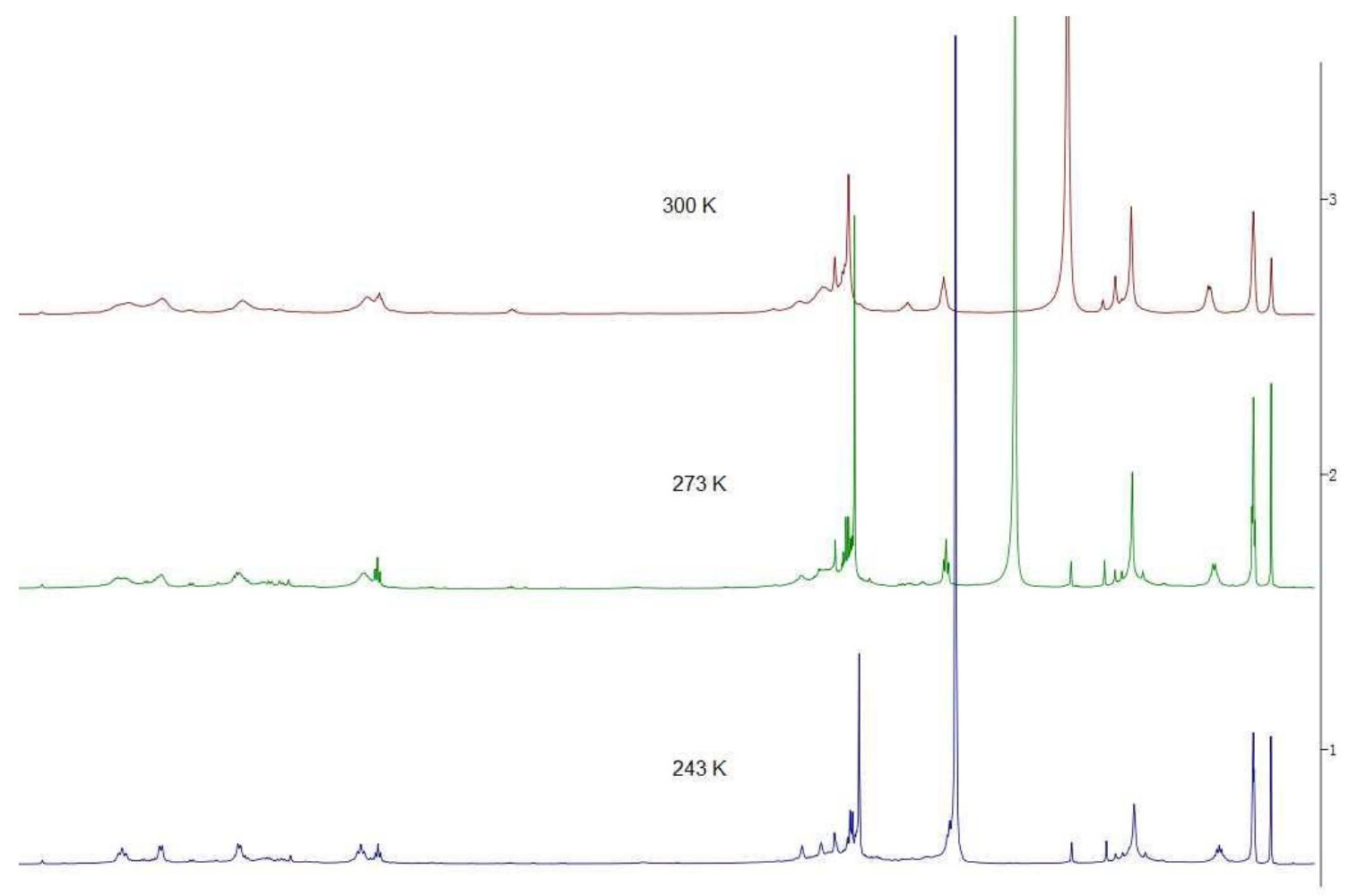

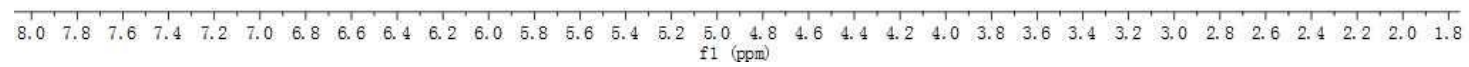


Fig. SI10. V-T proton spectrum of 4-QM in acetone- $d_{6}$.
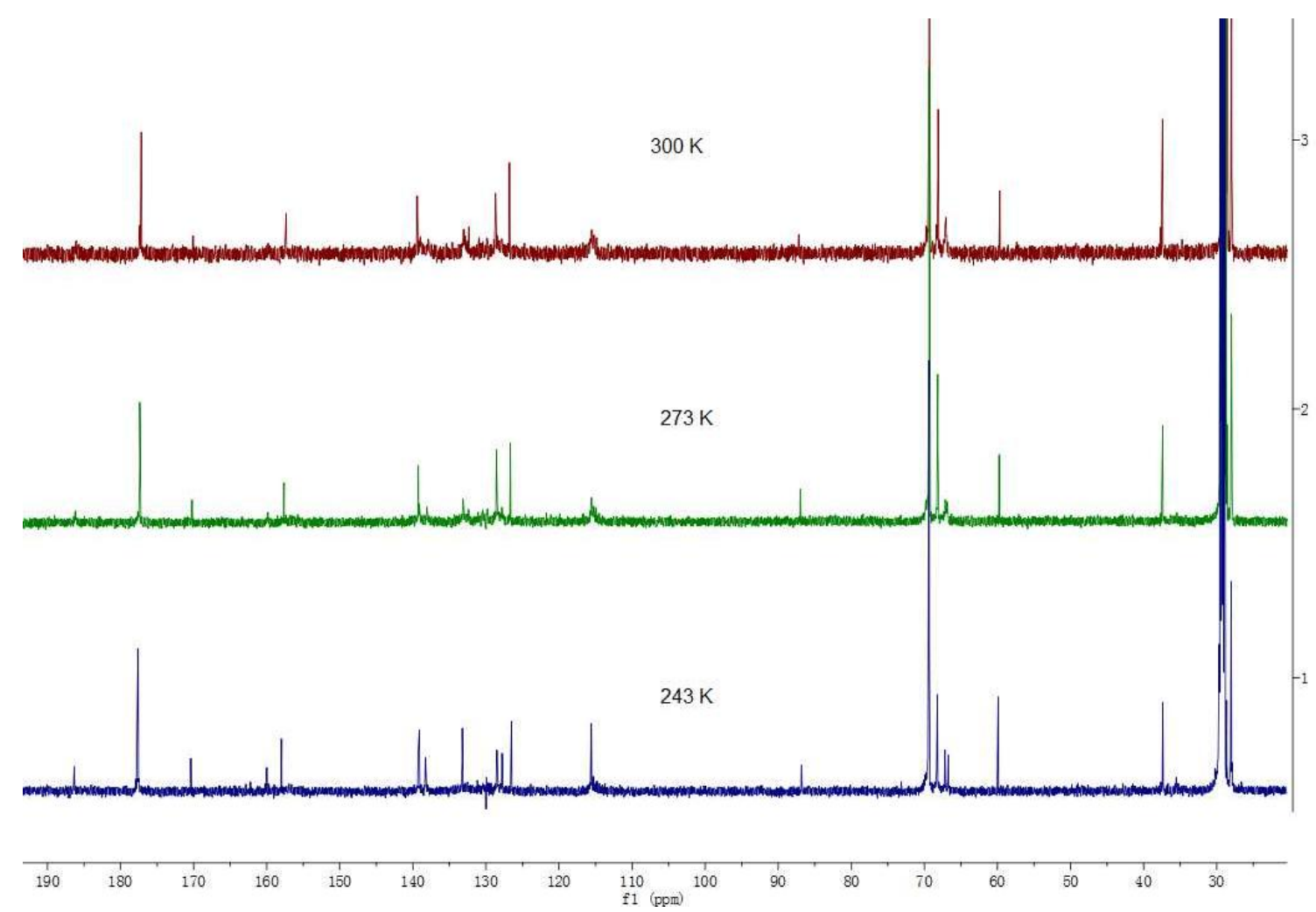

Fig. SI11. V-T carbon spectrum of 4-QM in acetone- $d_{6}$.
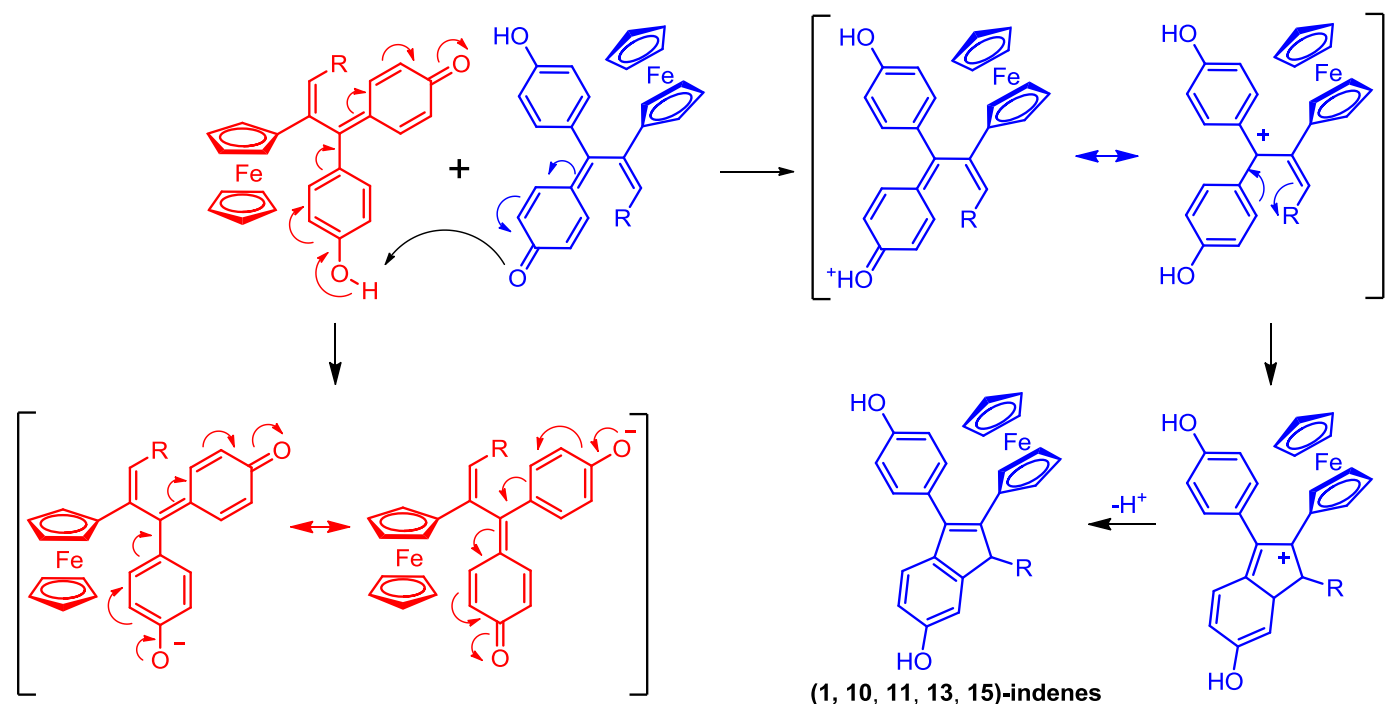

$(1,10,11,13,15)$-indenes

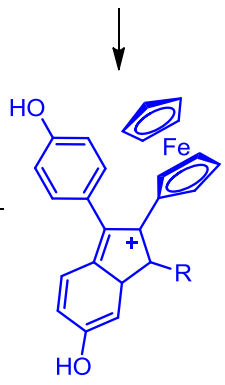

Fig. SI12. Proposed mechanism of interconversion of the neutral QMs and phenolates, and also formation of the ferrocenyl-indenes. The anionic charge so generated can be delocalized over both aryl rings; this also allows rotation about the $\mathrm{C}(1)-\mathrm{C}(2)$ bond, thereby equilibrating their NMR resonances. Meanwhile, the newly formed cation can be stabilized as a doubly benzylic system in which both aryl substituents possess para-hydroxyl substituents. However, over time, decomposition to form a ferrocenyl-indene became evident, a process readily 
understood as arising from a cyclization that places the positive charge adjacent to the strongly stabilizing ferrocenyl moiety; subsequent deprotonation yields the observed indenes.

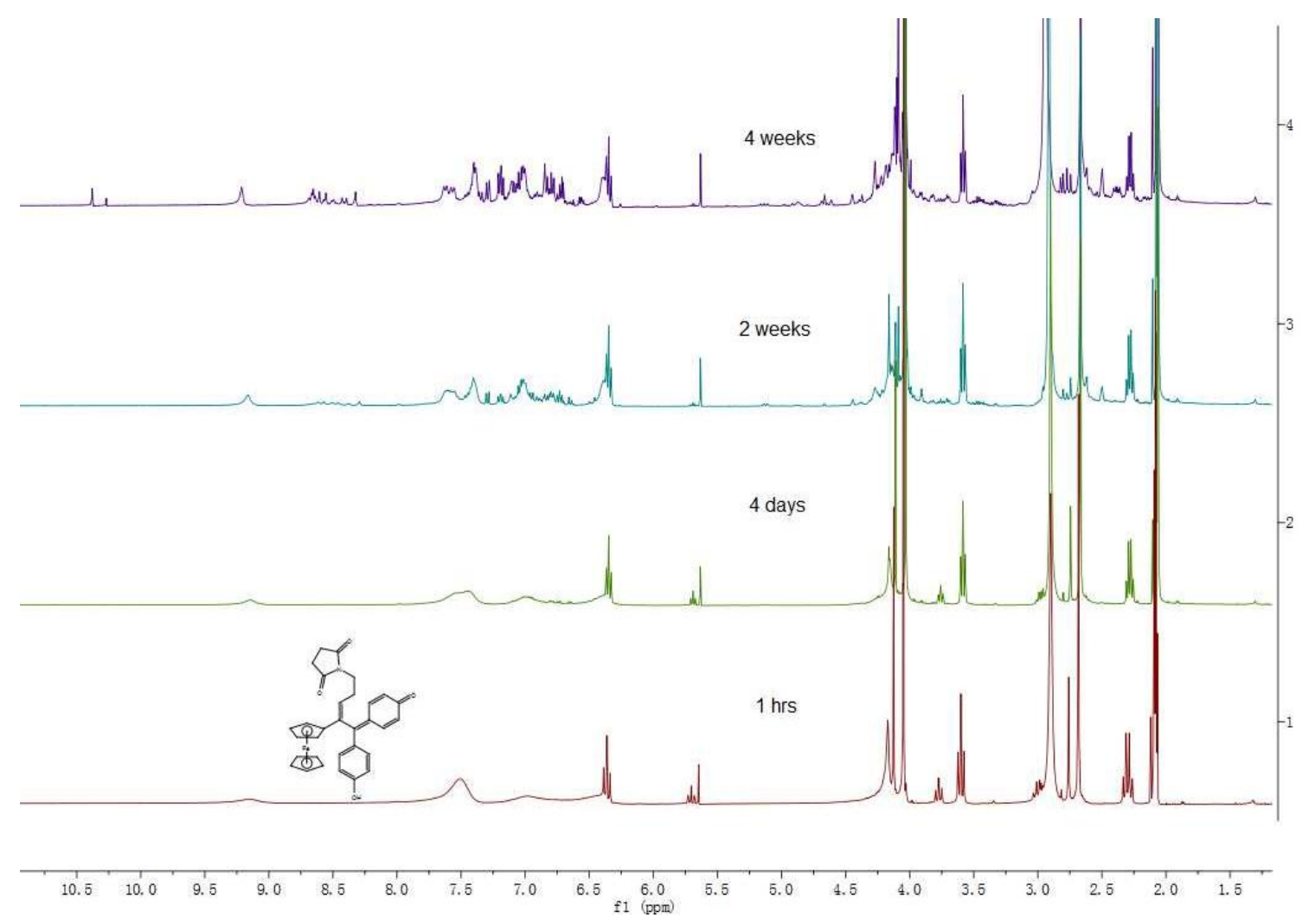

Fig. SI13. Stability spectrum of 4-QM in acetone- $d_{6}$. 

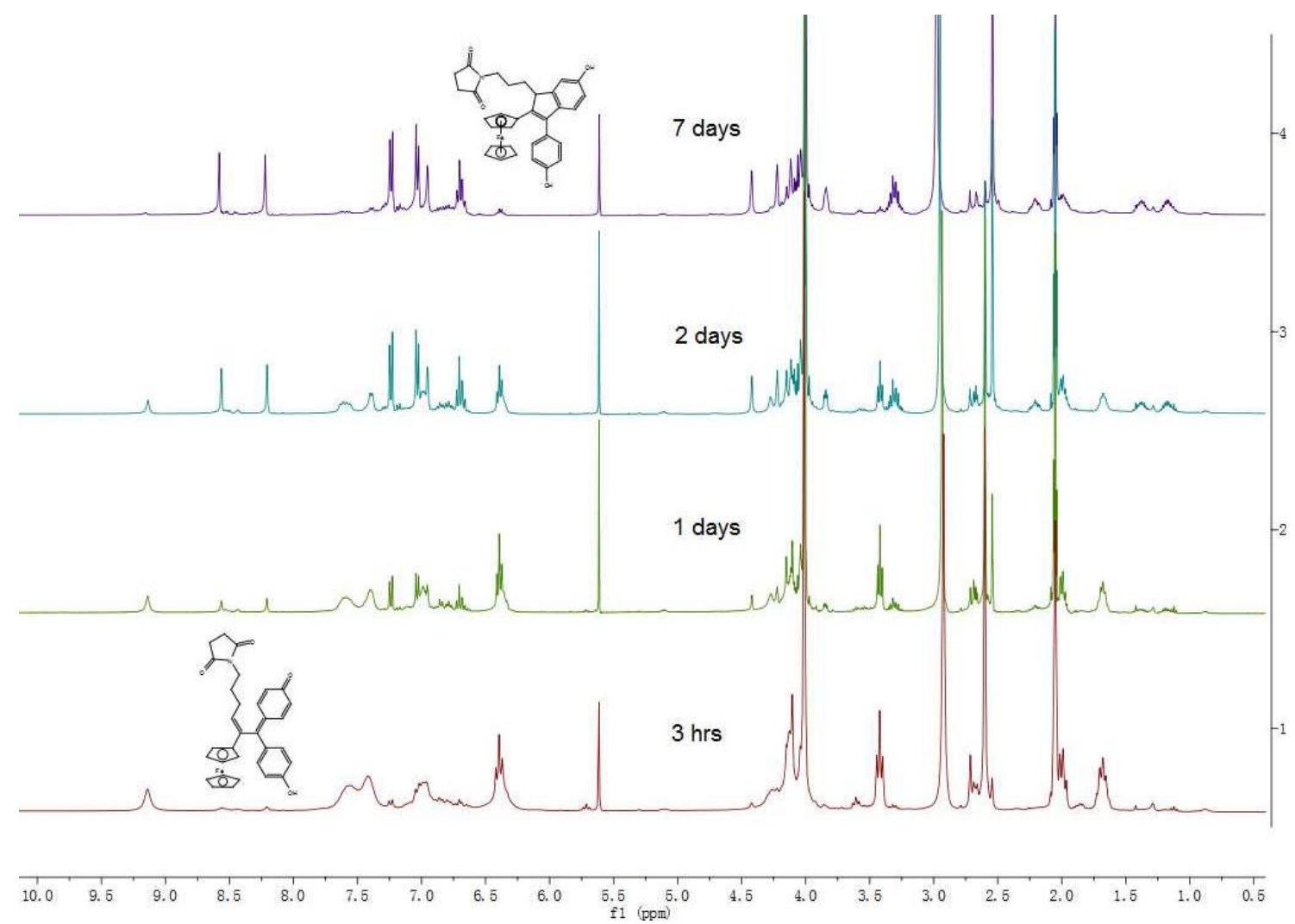

Fig. SI14. Stability spectrum of 10-QM in acetone- $d_{6}$. 

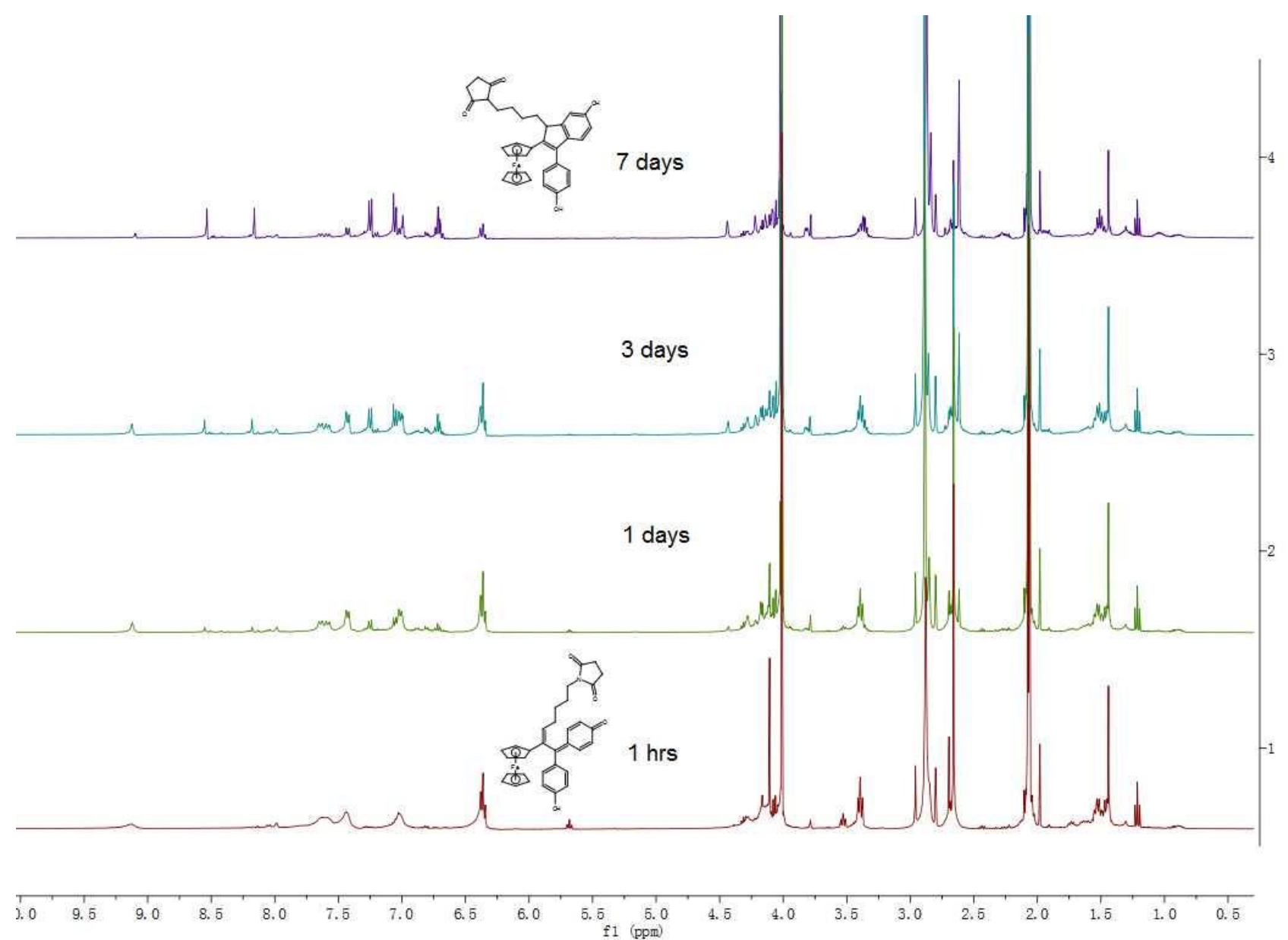

Fig. SI15. Stability spectrum of 11-QM in acetone- $d_{6}$. 

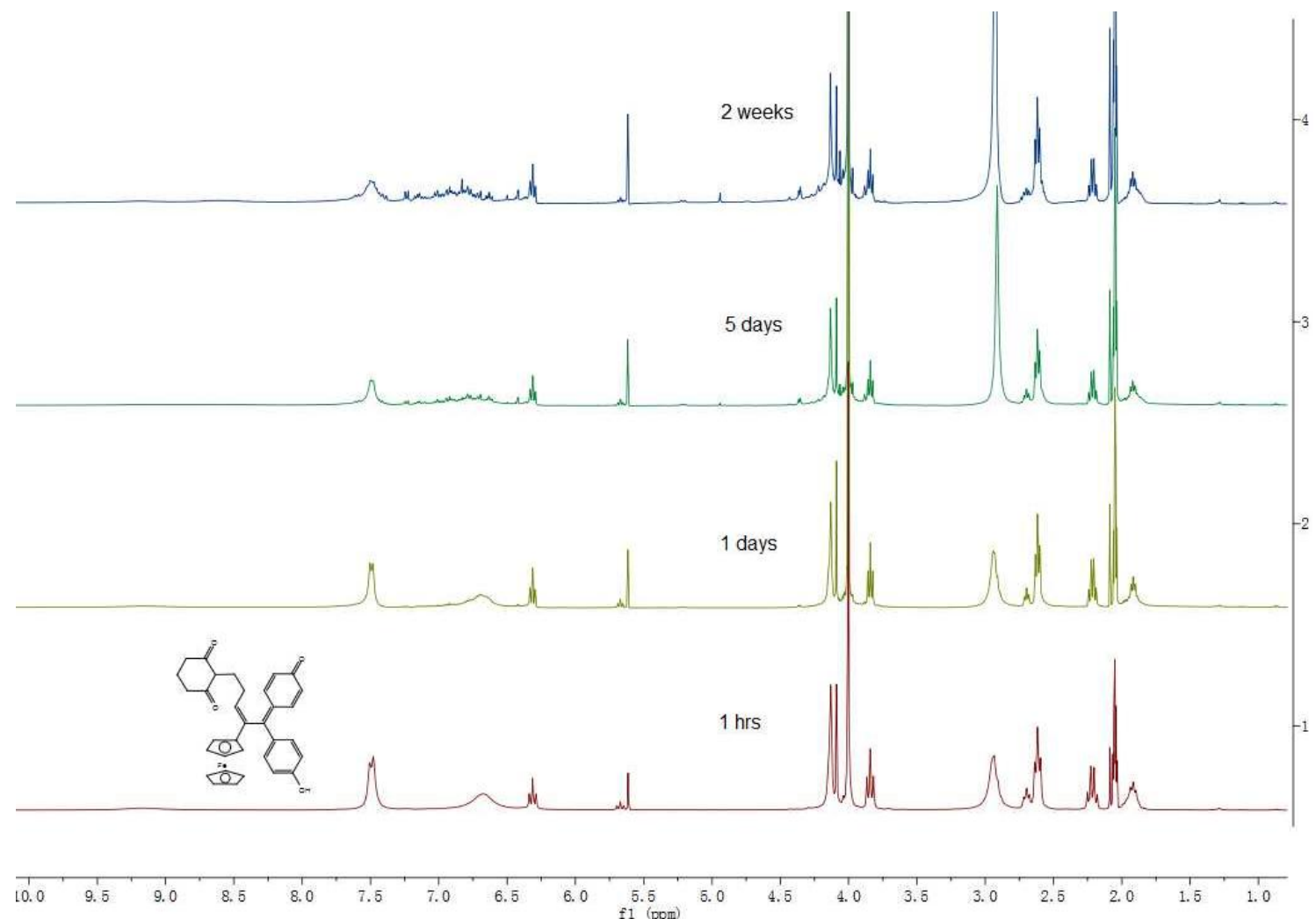

Fig. SI16. Stability spectrum of 12-QM in acetone- $d_{6}$. 


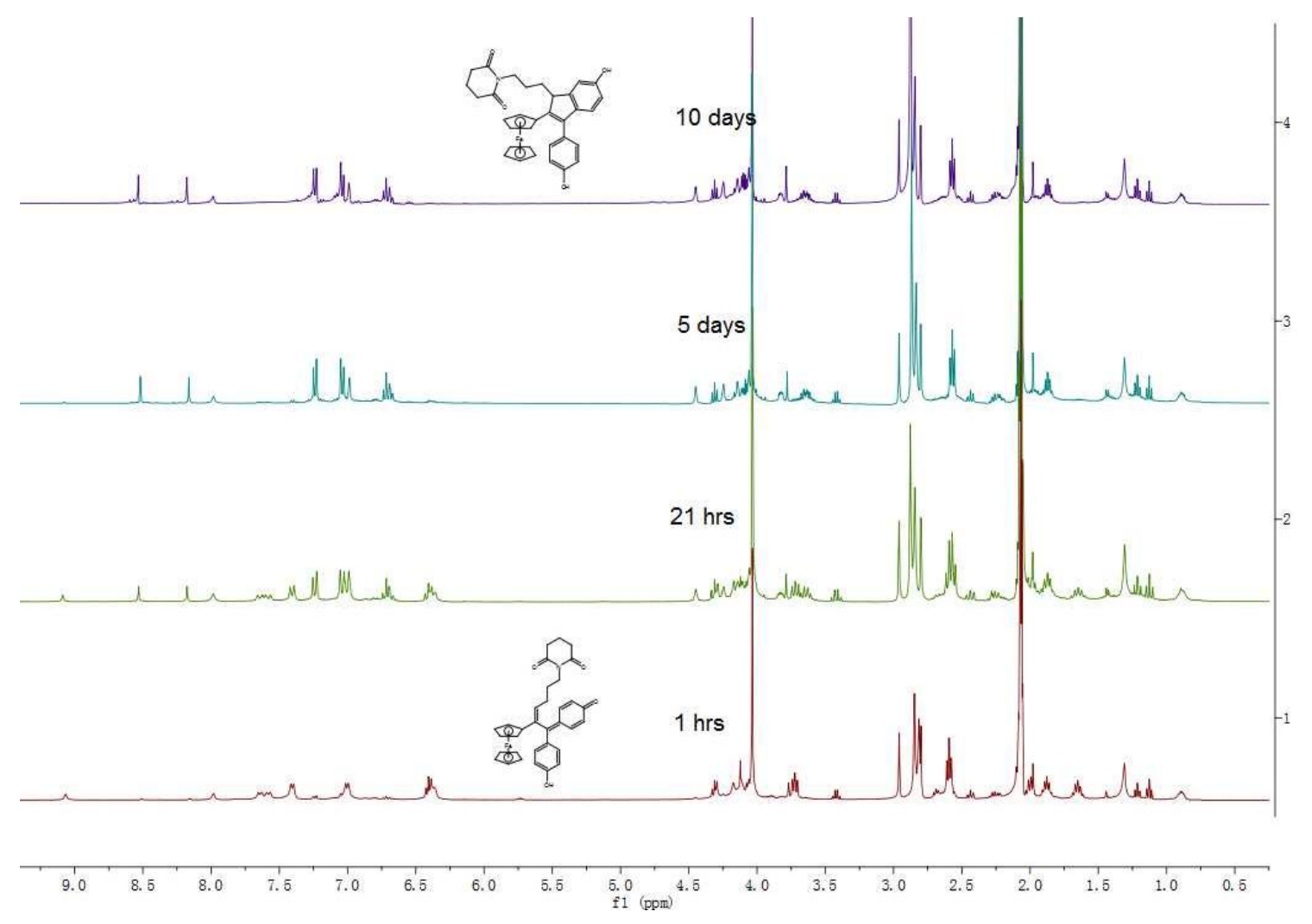

Fig. SI17. Stability spectrum of 13-QM in acetone- $d_{6}$. 

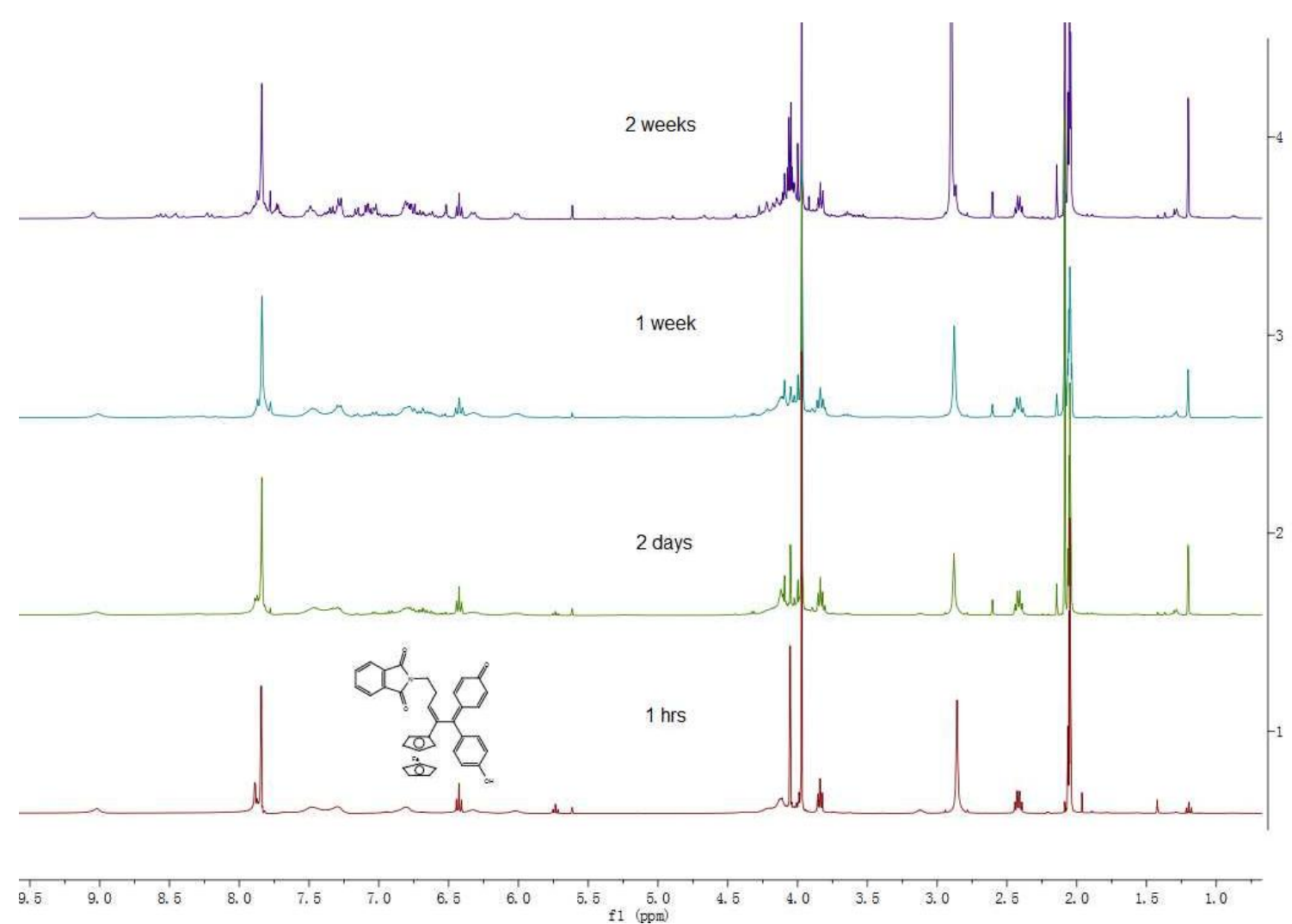

Fig. SI18. Stability spectrum of 14-QM in acetone- $d_{6}$.
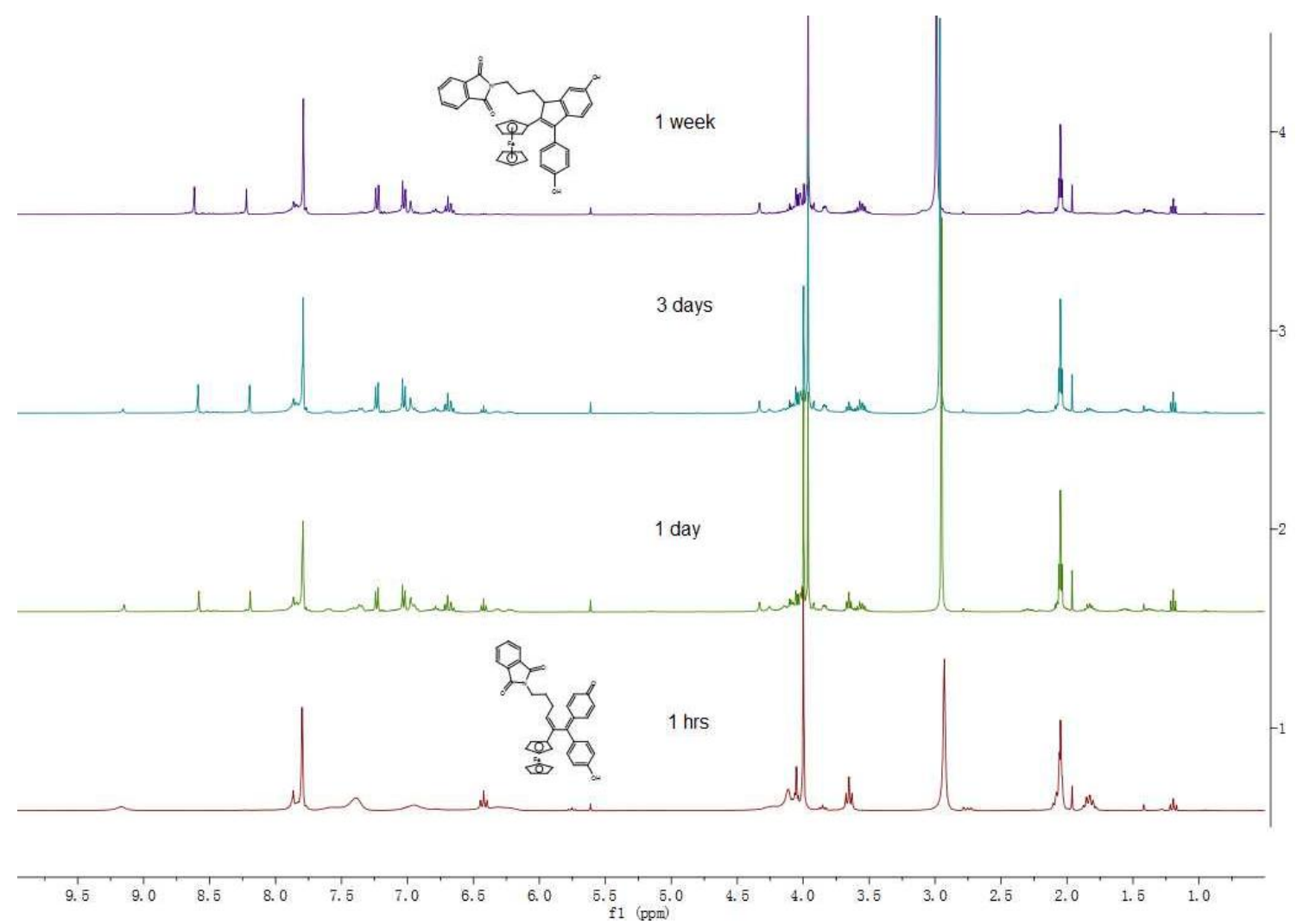
Fig. SI19. Stability spectrum of 15-QM in acetone- $d_{6}$.

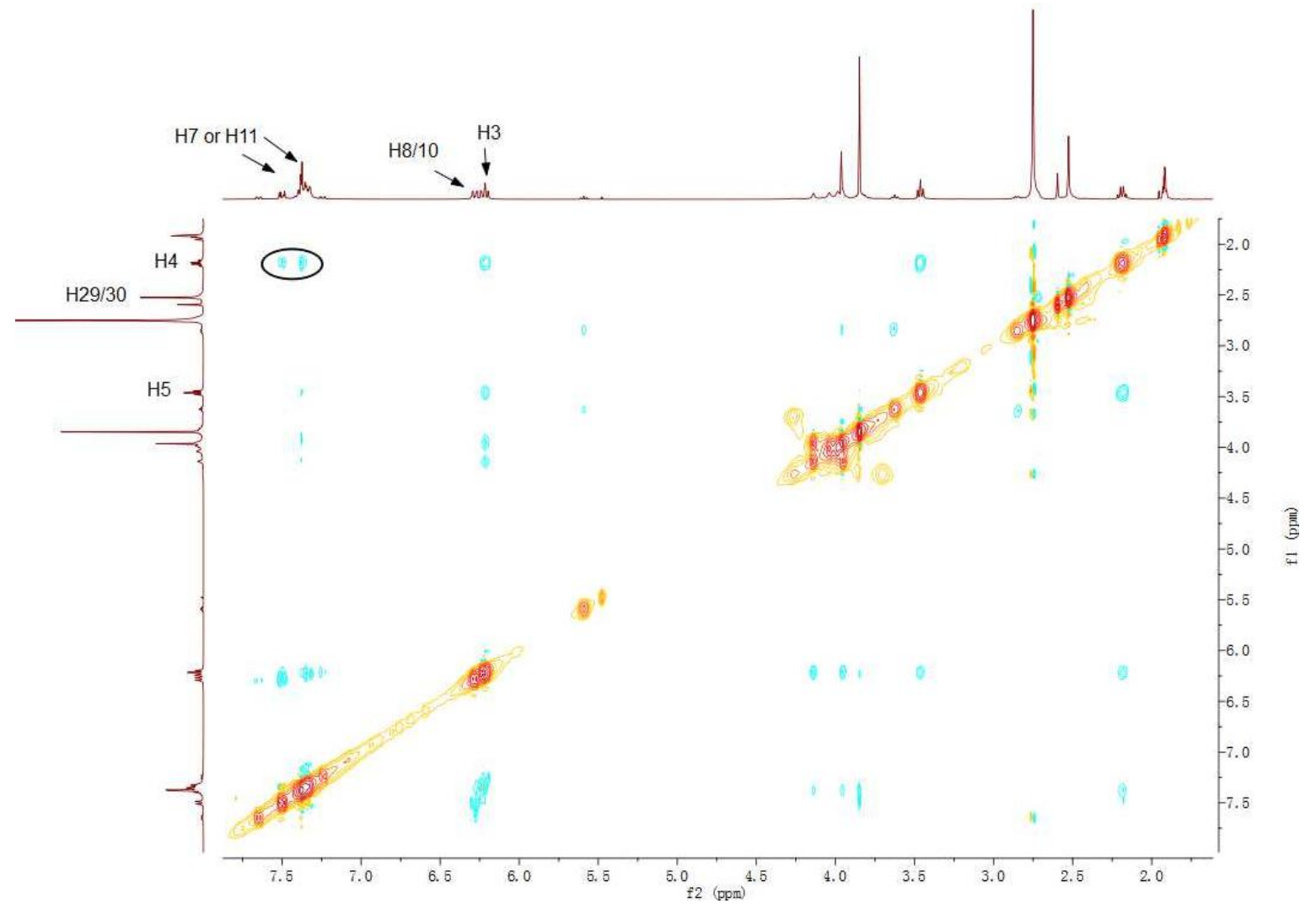

Fig. SI20. 2D-NOESY spectrum of $E-16-Q M$ in acetone- $d_{6}$. 


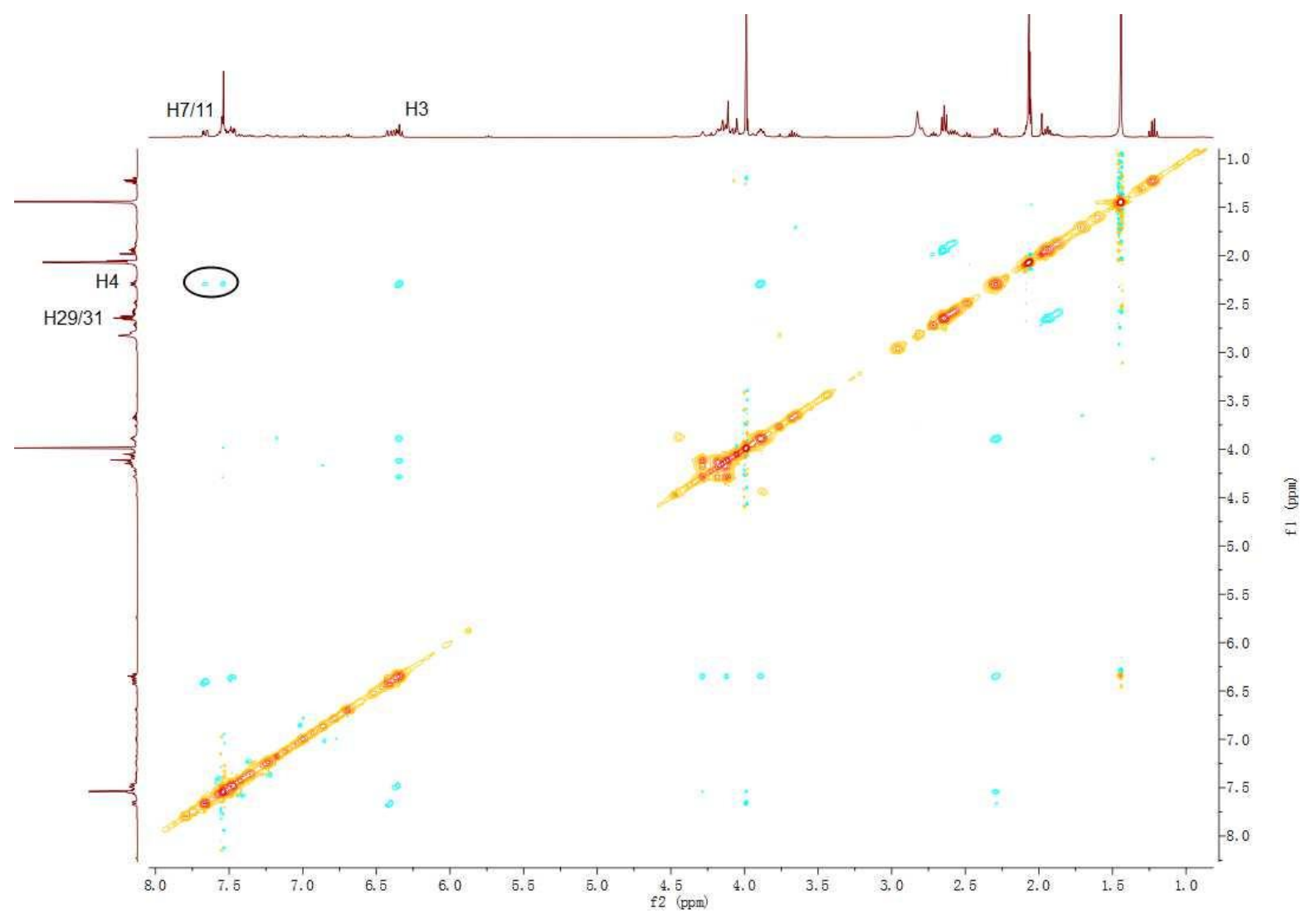

Fig. SI21. 2D-NOESY spectrum of $E-\mathbf{1 8 - Q M}$ in acetone- $d_{6}$. 


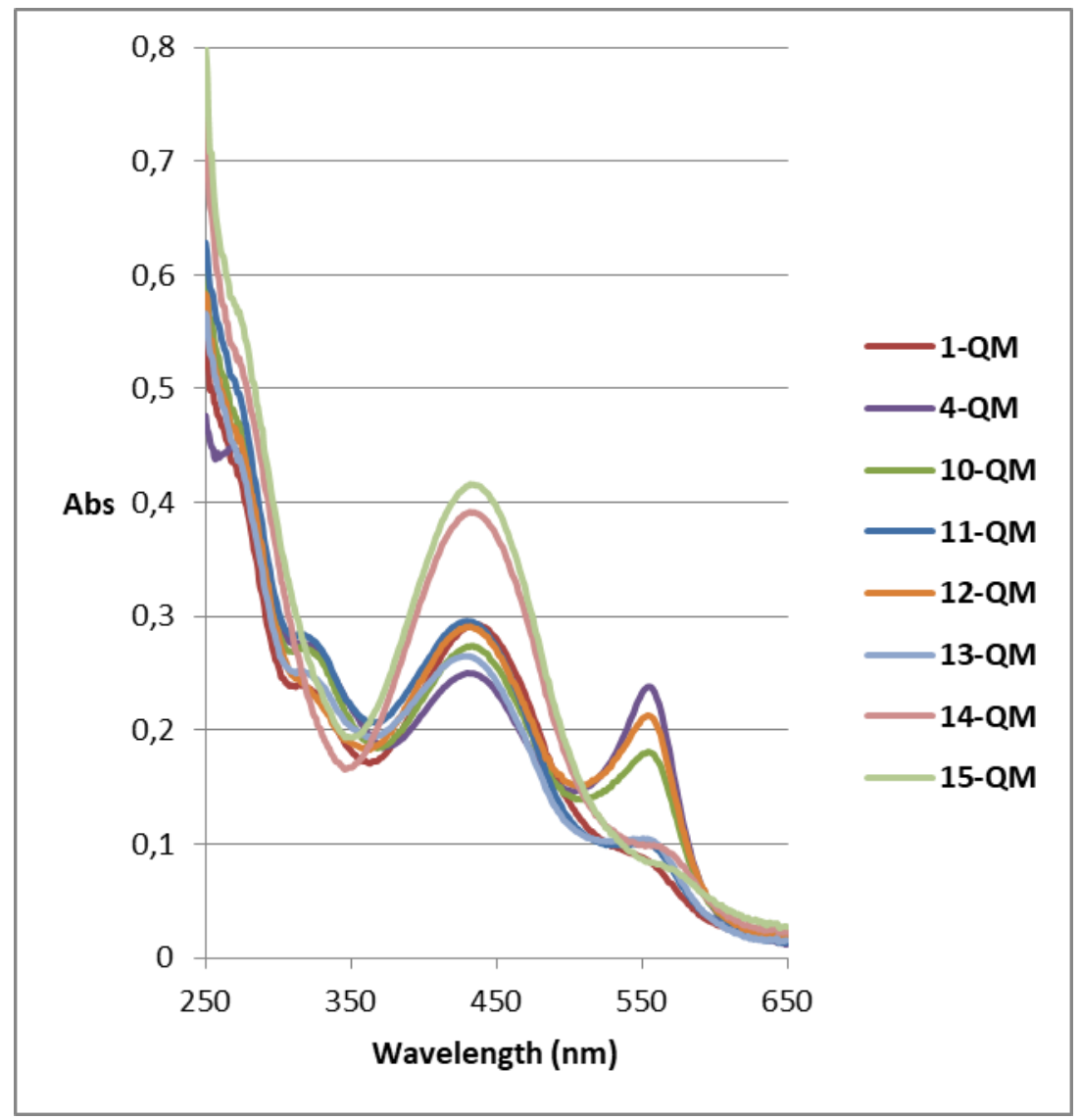

Fig. SI22. UV-Vis spectrum of QMs $(25 \mu \mathrm{M})$ observed in phosphate buffer at $37^{\circ} \mathrm{C}, \mathrm{pH}$ 7.4.

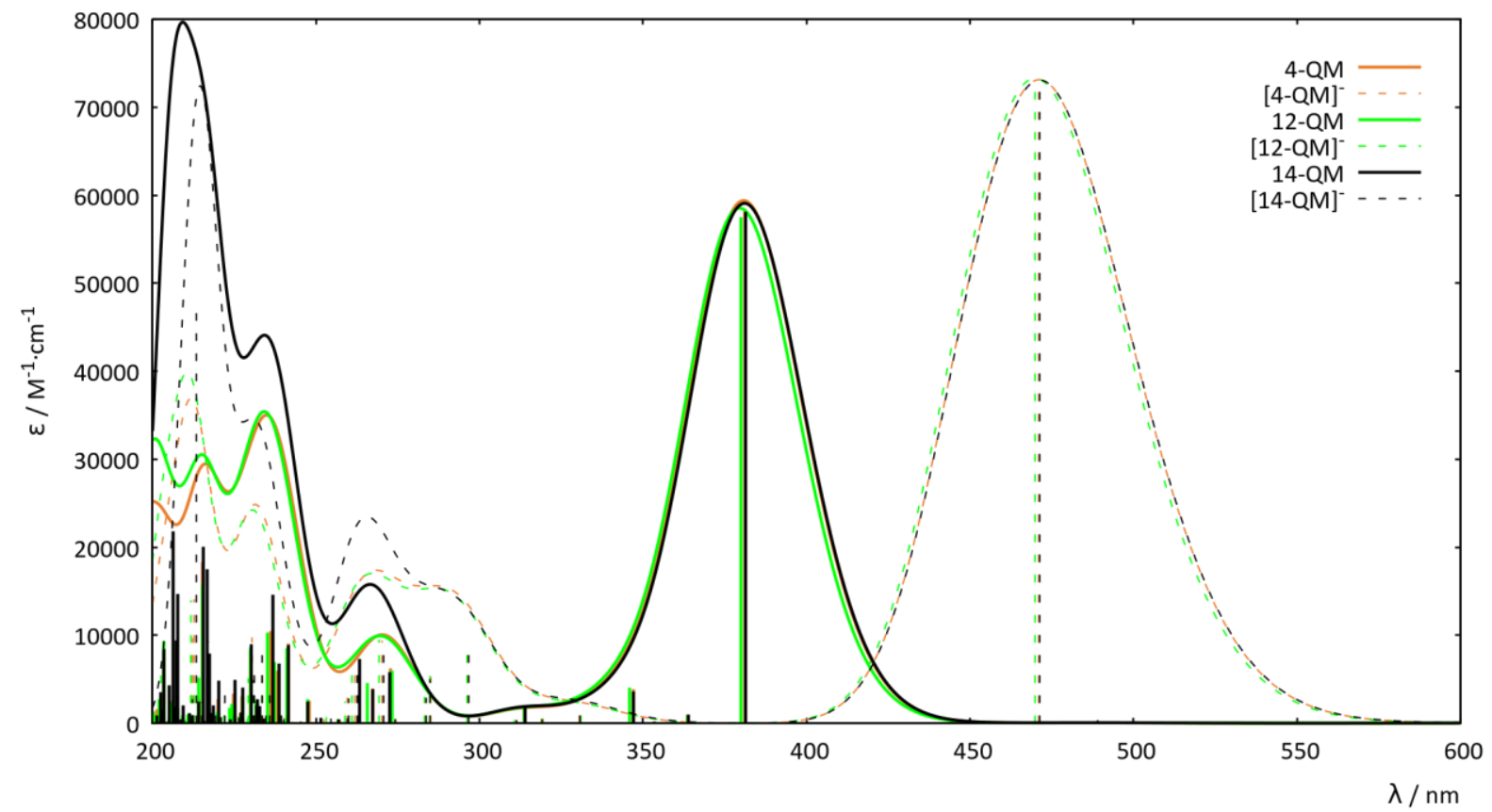


Fig. SI23. The UV-Vis absorption spectra of the conformer with the lp- $\pi$ interaction of the 4-QM, 12-QM and 14QM compounds (with the phenol protonated and deprotonated, as shown in Figure 5 of the main text) at the CAM-B3LYP/6-31+G* level of theory.

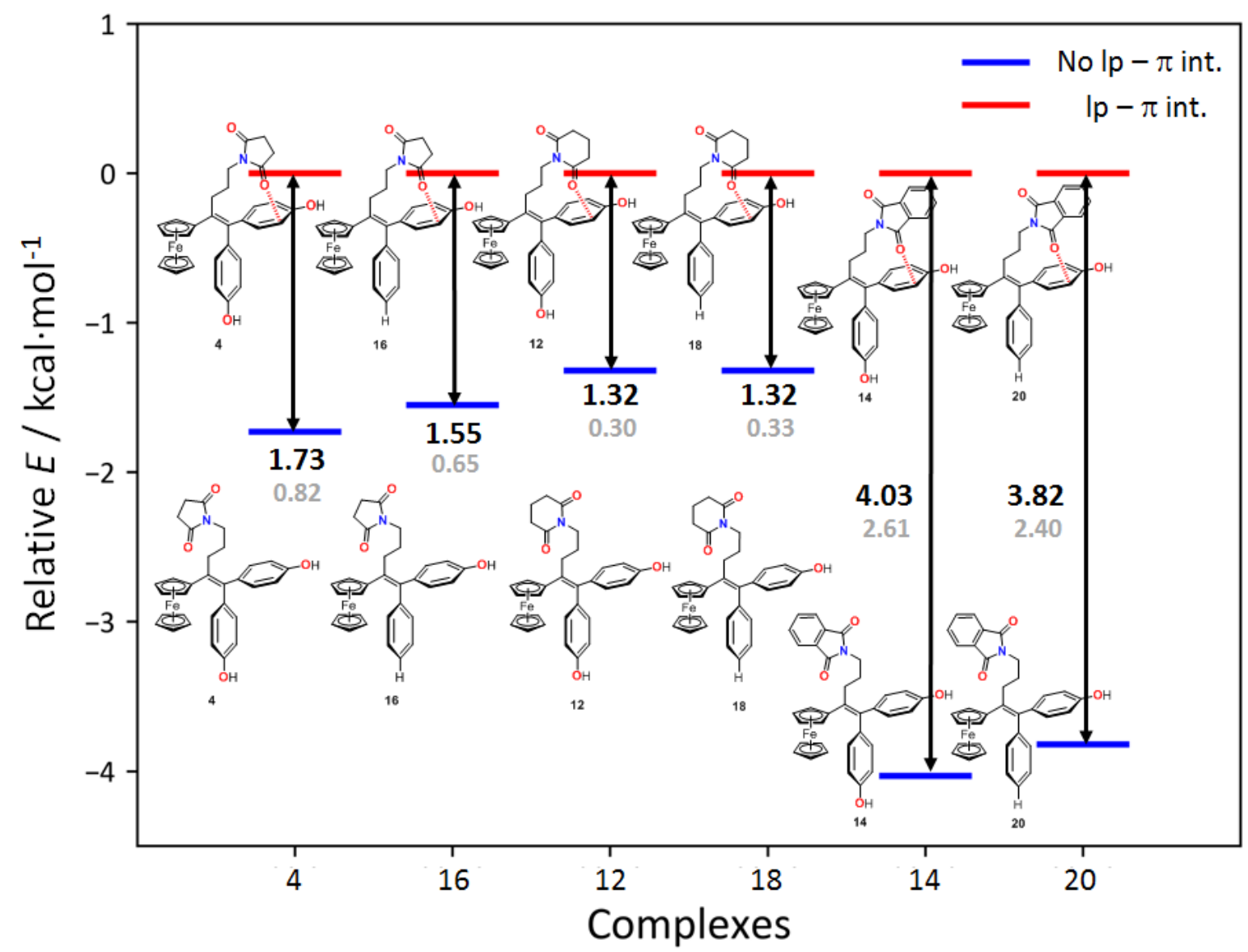

Fig. SI24. Relative energies of complexes 4, 16, 12, 18, 14 and 20, optimized at the B3LYP/6-31+G* level of theory with the D3-BJ Grimme's dispersion in acetone (PCM). Values in black represent single point calculations performed at the optimized structures at the B2LYPD3/6-31+G* level of theory in acetone (PCM). Values in grey represent single point calculations performed at the optimized structures at the PBEQIDH/6-31+G* level of theory in acetone (PCM). The $\mathrm{O}-\mathrm{C}_{\mathrm{Bz}}$ distances shown in red are more than $4 \AA$ I long. 


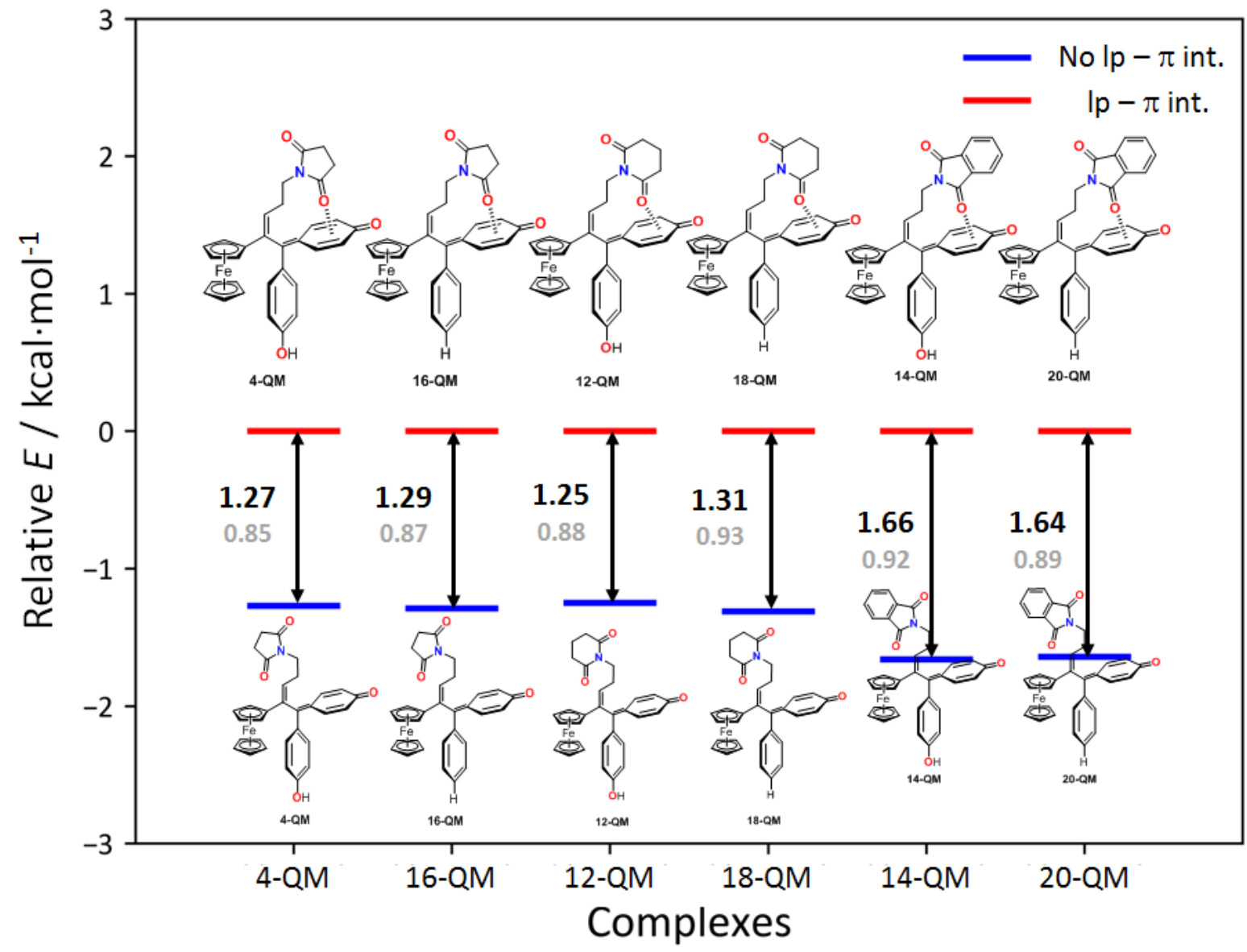

Fig. SI25. Relative energies of complexes 4-QM, 16-QM, 12-QM, 18-QM, 14-QM and 20-QM, optimized at the B3LYP/6-31+G* level of theory with the D3-BJ Grimme's dispersion in acetone (PCM). Values in black represent single point calculations performed at the optimized structures at the B2LYPD3/6-31+G* level of theory in acetone (PCM). Values in grey represent single point calculations performed at the optimized structures at the PBEQIDH/6-31+G* level of theory in acetone (PCM). 


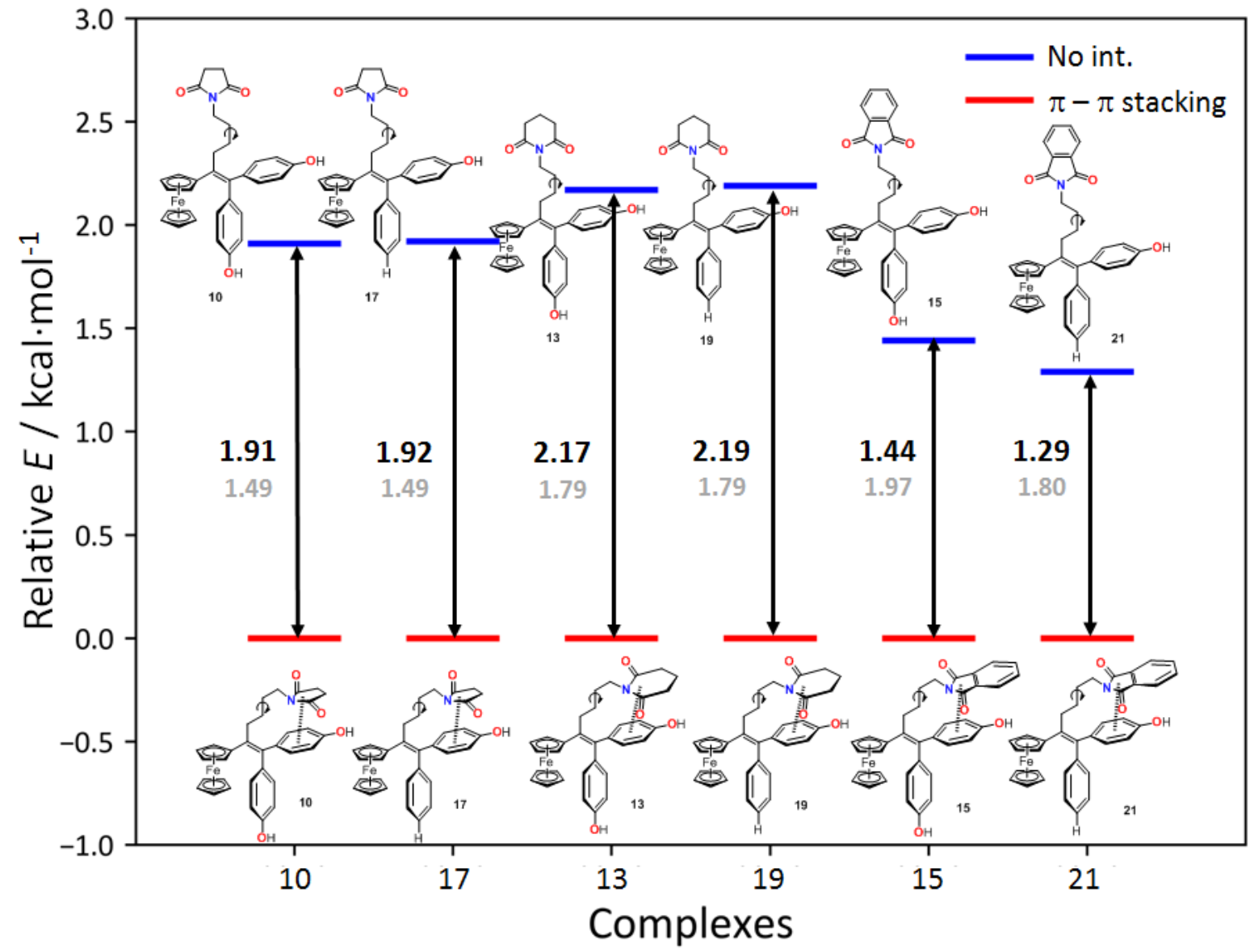

Fig. SI26. Relative energies of complexes 10, 17, 13, 19, 15 and 21, optimized at the B3LYP/6-31+G* level of theory with the D3-BJ Grimme's dispersion in acetone (PCM). Values in black represent single point calculations performed at the optimized structures at the B2LYPD3/6-31+G* level of theory in acetone (PCM). Values in grey represent single point calculations performed at the optimized structures at the PBEQIDH/6-31+G* level of theory in acetone (PCM). 


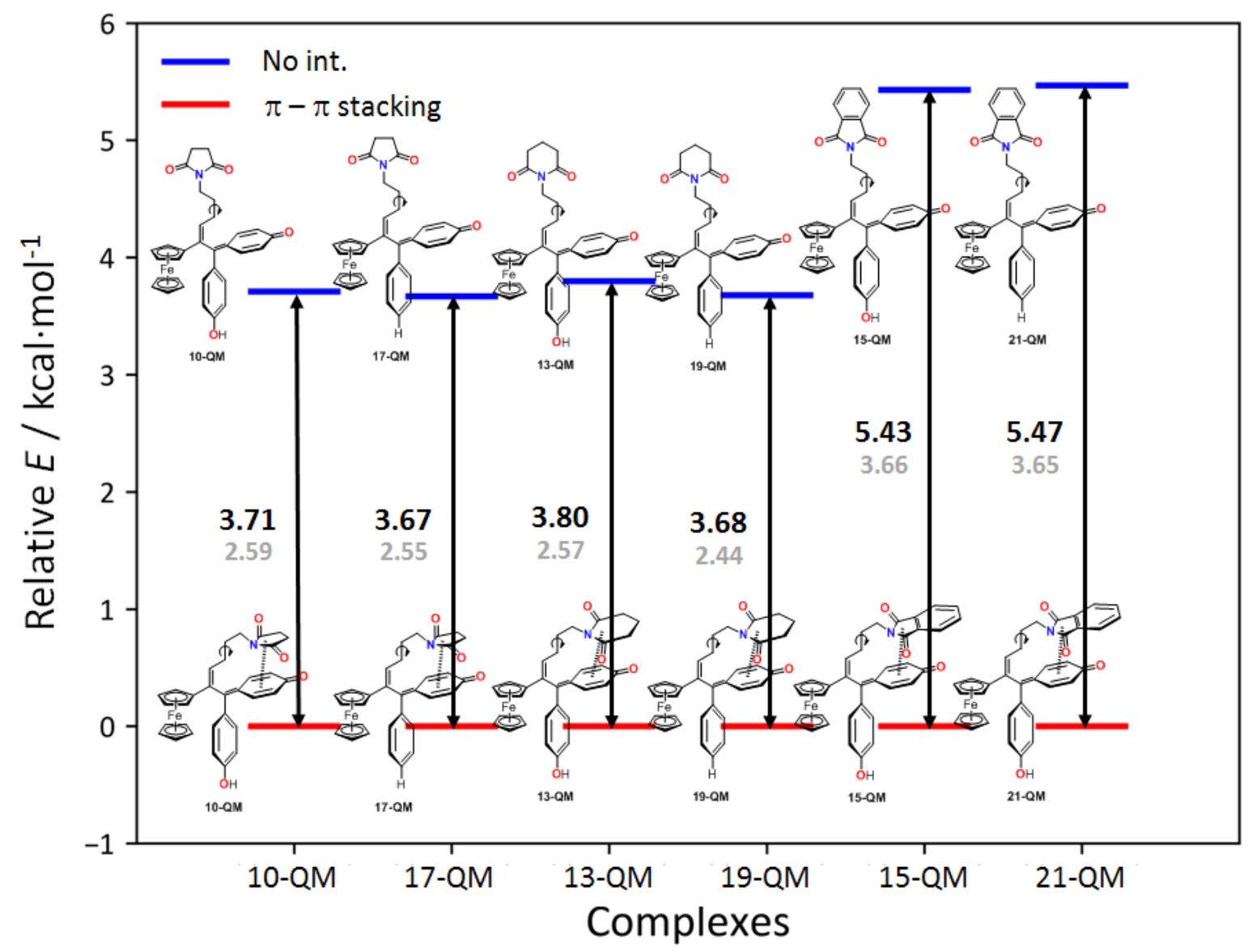

Fig. SI27. Relative energies of complexes 10-QM, 17-QM, 13-QM, 19-QM, 15-QM and 21-QM, optimized at the B3LYP/6-31+G* level of theory with the D3-BJ Grimme's dispersion in acetone (PCM). Values in black represent single point calculations performed at the optimized structures at the B2LYPD3/6-31+G* level of theory in acetone $(\mathrm{PCM})$. Values in grey represent single point calculations performed at the optimized structures at the PBEQIDH/6-31+G* level of theory in acetone (PCM). 


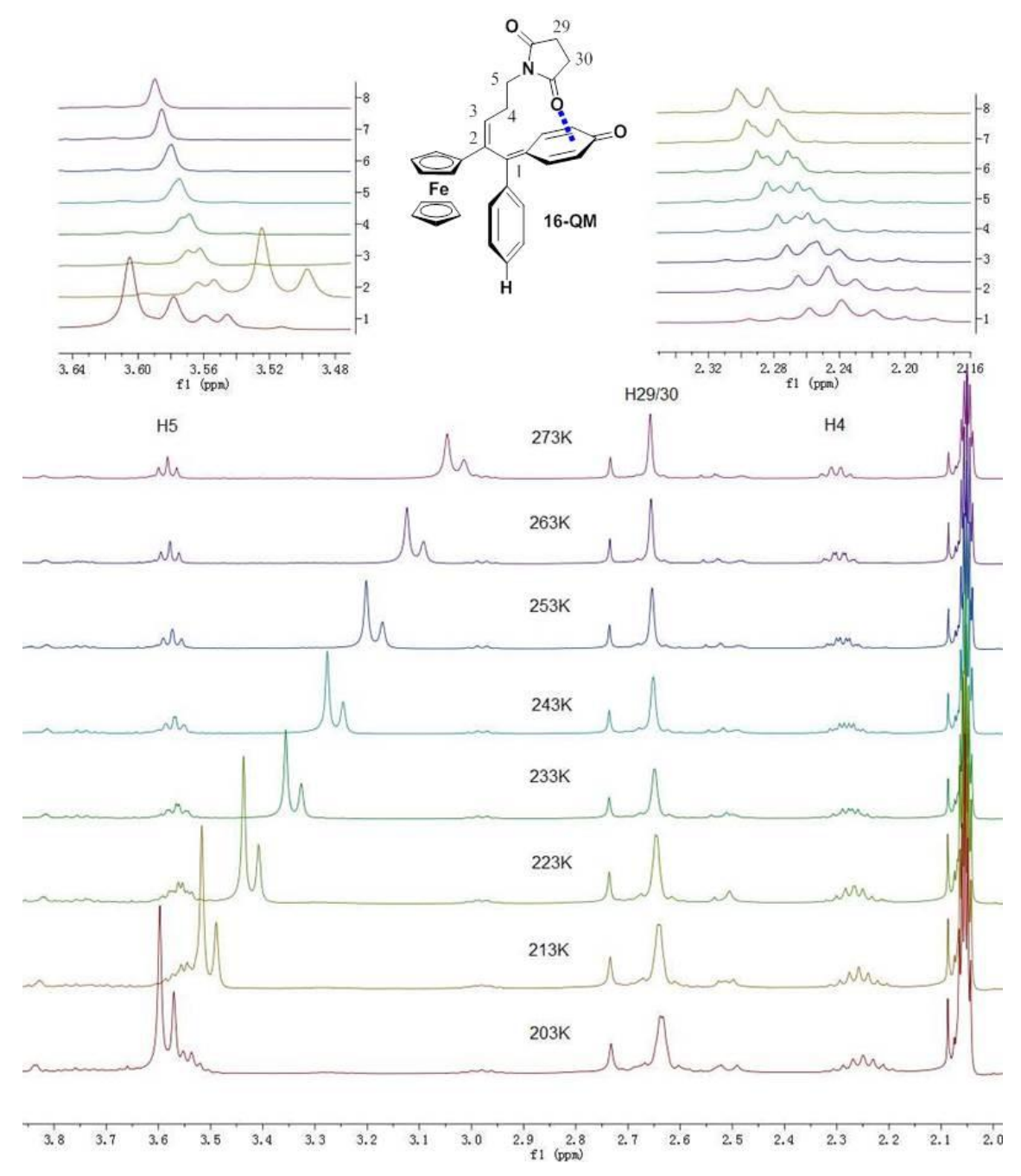

Fig. SI28. Variable temperature NMR data for 16-QM. The vinylic proton, H(3), appears as a $7 \mathrm{~Hz}$ triplet, while the adjacent methylene group at $\mathrm{C}(4)$ is a pseudo-quartet $(J=7 \mathrm{~Hz})$ arising from the doublet coupling from $\mathrm{H}(3)$ together with a $7 \mathrm{~Hz}$ triplet from the methylene protons at $\mathrm{C}(5)$; finally, this latter $\mathrm{CH}_{2}$ unit also appears as a $7 \mathrm{~Hz}$ triplet. However, when cooled gradually to $203 \mathrm{~K}$, the methylene protons at $\mathrm{C}(5)$ develop quartet character, while its neighbour at $\mathrm{C}(4)$ has clearly become a septet, with hints of smaller outer peaks. To simplify the situation, a number of selective proton decoupling experiments were performed on 16-QM (two magnified insets). Irradiation of the methylene protons at $\mathrm{C}(4)$ removed the vicinal coupling to their neighbours at $\mathrm{C}(5)$ which now appeared as a singlet; however, at $203 \mathrm{~K}$ the protons at $\mathrm{C}(5)$ were resolved into a pair of $13 \mathrm{~Hz}$ doublets. Analogously, irradiation of the methylene protons at $\mathrm{C}(5)$ initially caused the $\mathrm{CH}_{2}$ group at $\mathrm{C}(4)$ to appear as a $7 \mathrm{~Hz}$ doublet, but at low temperature it appeared as two pairs of doublets of doublets $\left({ }^{2} J_{\mathrm{HH}}=14.7 \mathrm{~Hz},{ }^{3} J_{\mathrm{HH}}=7 \mathrm{~Hz}\right)$. 
Table SI1. NTOs of the first brightest transition in the TD-DFT spectra for complexes 4-QM, 12-QM, 14-QM and their respective phenolic deprotonated forms.

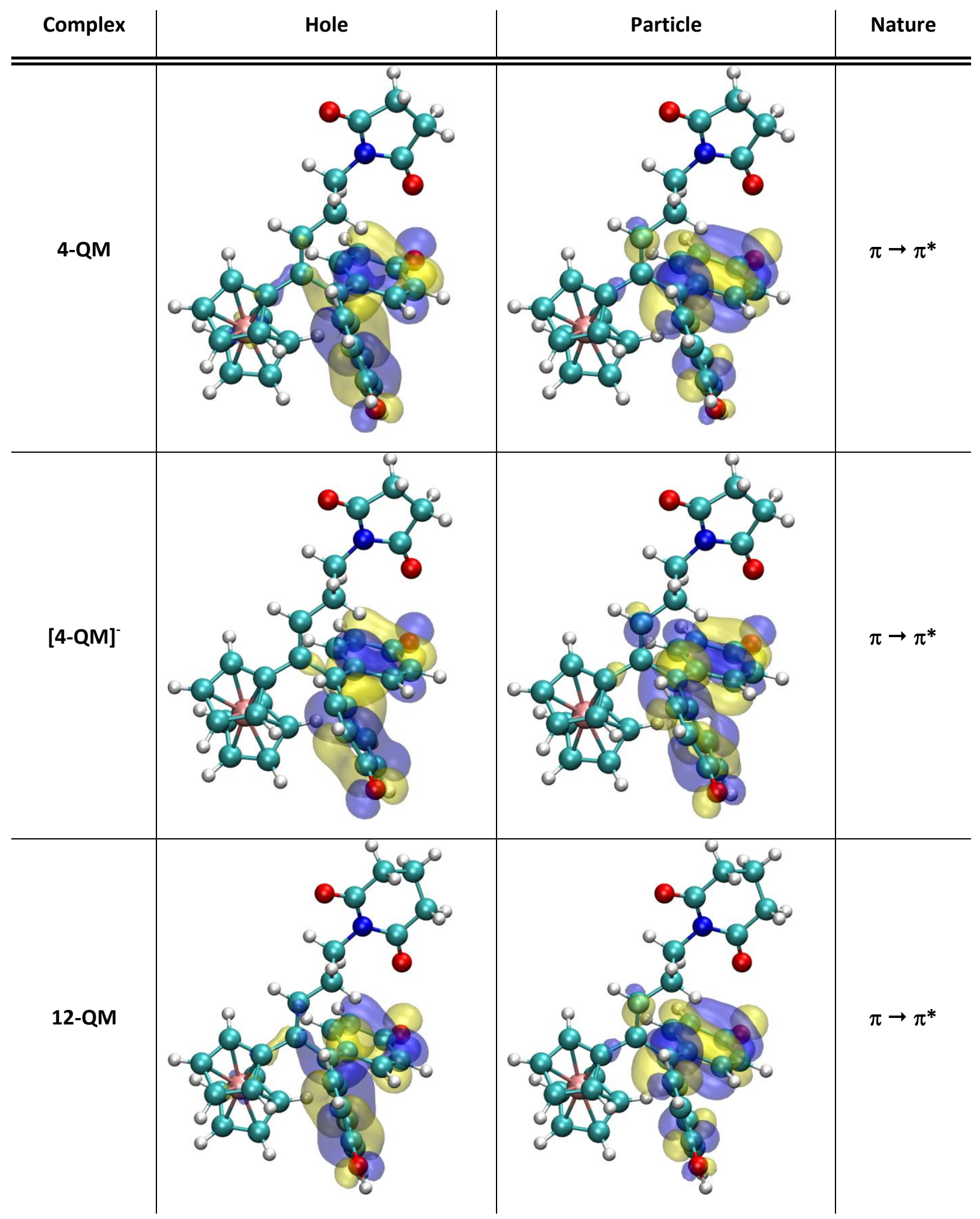




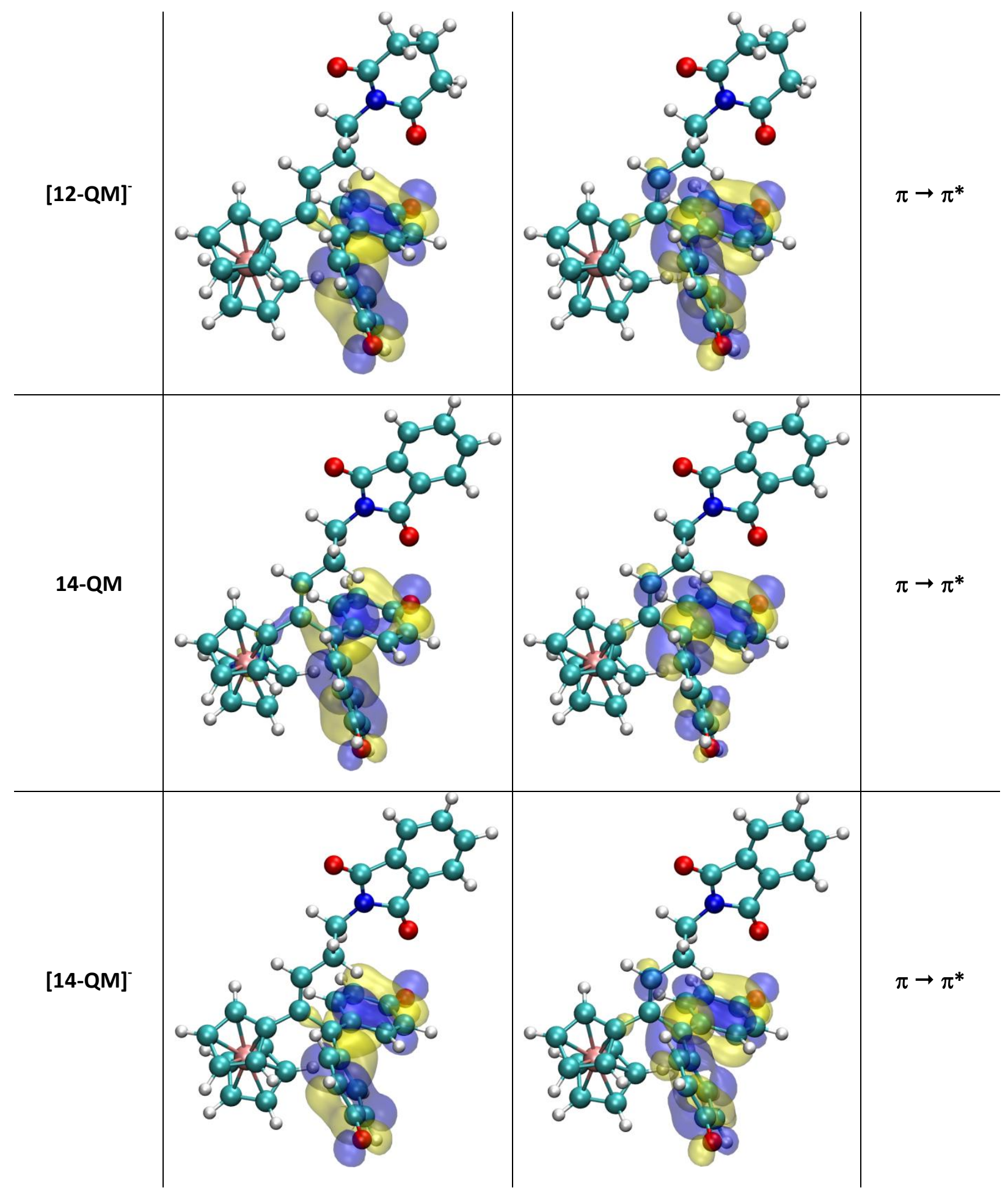

Table SI2. The half-life of selected compounds $(25 \mu \mathrm{M})$ measured at $37^{\circ} \mathrm{C}$ in phosphate buffer at pH 6.0 and 5.0. 


\begin{tabular}{ccc}
\hline Comp. & $\mathrm{T}_{1 / 2}$ at $\mathrm{pH} 5.0$ (mins) & $\begin{array}{c}\mathrm{T}_{1 / 2} \text { at } \mathrm{pH6.0} \\
\text { (mins) }\end{array}$ \\
\hline 1-QM & 10.9 & 27.2 \\
4-QM & 44.2 & 203.9 \\
$\mathbf{1 0 - Q M}$ & 13.1 & 30.5 \\
$\mathbf{1 1 - Q M}$ & 17.3 & 31.9 \\
$\mathbf{1 2 - Q M}$ & 38.5 & 113.6 \\
$\mathbf{1 3 - Q M}$ & 19.2 & 27.3 \\
\hline
\end{tabular}

(a) The rates of disappearance of QMs at around $430 \mathrm{~nm}$ as described in experimental section.

Table SI3. In Vitro testing results for 4 from the NCI/DTP, data from one experiment shown, maximum concentration: $100 \mu \mathrm{M}$, after $48 \mathrm{~h}$ incubation. 


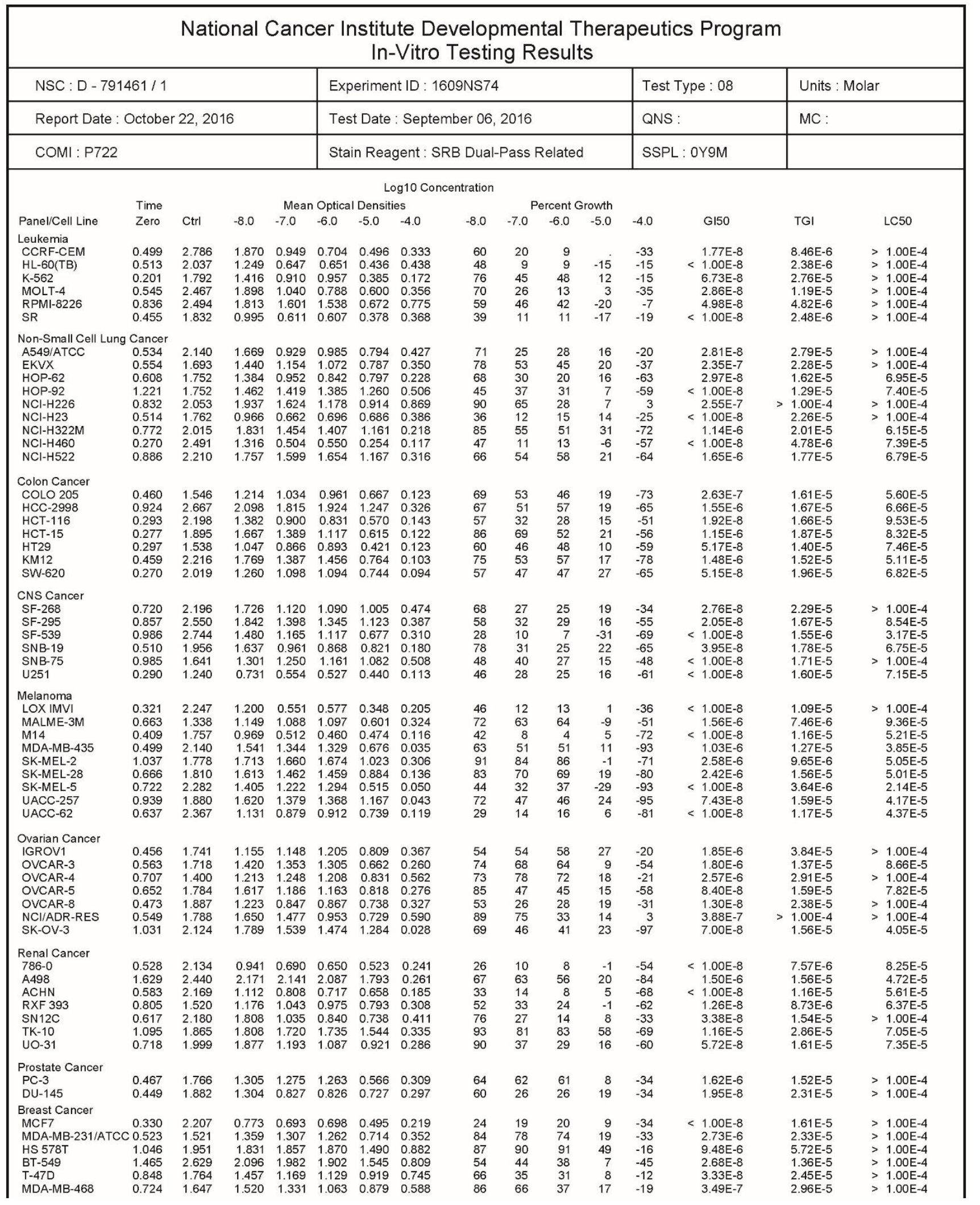

Table SI4. Rotation barriers found for 16-QM at the B3LYP-D3BJ/6-31+G* level of theory. 


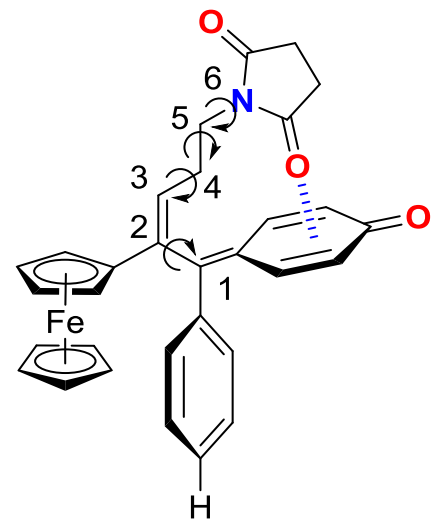

\begin{tabular}{|c|c|}
\hline Estimated Energy Barriers (kcal/mol) & B3LYP-D3BJ/6-31+G* \\
\hline C1 - C2 & 20.6 \\
\hline C3 - C4 & 1.9 \\
\hline C4 - C5 & 3.95 \\
\hline C5 - N6 & 5.0 \\
\hline
\end{tabular}


Table SI5. Crystallographic Data.

\begin{tabular}{|c|c|c|c|c|c|c|}
\hline Compound & 14 & $Z-16$ & 17 & $Z-20$ & $E-4-\mathrm{QM}$ & $E-12-\mathrm{QM}$ \\
\hline Formula & $\mathrm{C}_{38} \mathrm{H}_{35} \mathrm{FeNO}_{5}$ & $\mathrm{C}_{31} \mathrm{H}_{29} \mathrm{FeNO}_{3}$ & $\mathrm{C}_{32} \mathrm{H}_{31} \mathrm{FeNO}_{3}$ & $\mathrm{C}_{38} \mathrm{H}_{35} \mathrm{FeNO}_{4}$ & $\mathrm{C}_{32.5} \mathrm{H}_{30} \mathrm{FeNO}_{4.5}$ & $\mathrm{C}_{33.5} \mathrm{H}_{32} \mathrm{FeNO}_{4.5}$ \\
\hline$F . W .\left[\mathrm{g} \mathrm{mol}^{-1}\right]$ & 641.52 & 519.40 & 533.43 & 625.52 & 562.42 & 576.45 \\
\hline Crystal system & Triclinic & Monoclinic & Monoclinic & Triclinic & Monoclinic & Monoclinic \\
\hline Space group & $P-1$ & $P 21 / c$ & $P 21 / c$ & $P-1$ & $P 2_{1} / c$ & $C 2 / c$ \\
\hline$a[\AA]$ & $9.0292(3)$ & $11.5642(5)$ & $9.9878(3)$ & $9.1620(5)$ & $16.3306(7)$ & $34.0540(10)$ \\
\hline$b[\AA]$ & $12.4968(4)$ & $24.4529(12)$ & $18.1152(6)$ & $12.7172(6)$ & $8.6413(3)$ & $8.8249(2)$ \\
\hline$c[\AA]$ & $15.9071(6)$ & $8.9855(5)$ & $14.6303(5)$ & $14.8339(7)$ & 18.9232(9) & $19.0317(5)$ \\
\hline$\alpha\left[^{\circ}\right]$ & $111.067(2)$ & 90 & 90 & $66.866(2)$ & 90 & 90 \\
\hline$\beta\left[^{\circ}\right]$ & $95.140(2)$ & $103.184(3)$ & $98.352(2)$ & $83.823(2)$ & $99.148(2)$ & $105.934(2)$ \\
\hline$\gamma\left[^{\circ}\right]$ & $101.789(2)$ & 90 & 90 & $84.694(2)$ & 90 & 90 \\
\hline$V\left[\AA^{3}\right]$ & $1613.27(10)$ & $2473.9(2)$ & $2619.00(15)$ & $1577.78(14)$ & 2636.43(19) & $5499.7(3)$ \\
\hline$Z$ & 2 & 4 & 4 & 2 & 4 & 8 \\
\hline$T[\mathrm{~K}]$ & $200(1)$ & $200(1)$ & $200(1)$ & $200(1)$ & $200(1)$ & $200(1)$ \\
\hline$\lambda[\AA]$ & 0.71073 & 0.71073 & 0.71073 & 0.71073 & 0.71073 & 0.71073 \\
\hline GOF & 1.036 & 1.039 & 1.045 & 1.009 & 1.024 & 1.035 \\
\hline
\end{tabular}




\section{Reference}

(1) Liu, H.; Liu, J.; van Breemen, R. B.; Thatcher, G. R. J.; Bolton, J. L., Chem. Res. Toxicol. 2005, 18, 162.

(2) L. Palatinus, G. Chapuis, Journal of Applied Crystallography 2007, 40, 786-790.

(3) G. M. Sheldrick, Acta Crystallographica Section C 2015, 71, 3-8.

(4) L. J. Farrugia, Journal of Applied Crystallography 1999, 32, 837-838.

(5) O. V. Dolomanov, L. J. Bourhis, R. J. Gildea, J. A. K. Howard, H. Puschmann, Journal of Applied Crystallography 2009, 42, 339-341.

(6) Frisch M. J., et al.; Gaussian, Inc.: Wallingford, CT, 2017.

(7) Lee, C.; Yang, W.; Parr, R. G. Development of the Colle-Salvetti Correlation-Energy Formula into a Functional of the Electron Density. Physical Review B 1988, 37 (2), 785.

(8) Becke, A. D. Density-Functional Thermochemistry. III. The Role of Exact Exchange. The Journal of Chemical Physics 1993, 98 (7), 5648.

(9) Grimme, S.; Ehrlich, S.; Goerigk, L. Effect of the Damping Function in Dispersion Corrected Density Functional Theory. J. Comput. Chem. 2011, 32 (7), 1456-1465.

(10) Tomasi, J.; Mennucci, B.; Cammi, R. Quantum Mechanical Continuum Solvation Models. Chem. Rev. 2005, 105 (8), 2999-3094.

(11) Hehre, W. J.; Ditchfield, R.; Pople, J. A. Self-Consistent Molecular Orbital Methods. XII. Further Extensions of Gaussian-Type Basis Sets for Use in Molecular Orbital Studies of Organic Molecules. The Journal of Chemical Physics 1972, 56 (5), 2257-2261.

(12) Hariharan, P. C.; Pople, J. A. The Influence of Polarization Functions on Molecular Orbital Hydrogenation Energies. Theoret. Chim. Acta 1973, 28 (3), 213-222.

(13) Francl, M. M.; Pietro, W. J.; Hehre, W. J.; Binkley, J. S.; Gordon, M. S.; DeFrees, D. J.; Pople, J. A. Self- consistent Molecular Orbital Methods. XXIII. A Polarization- type Basis Set for Second- row Elements. The Journal of Chemical Physics 1982, 77 (7), 36543665 .

(14) Dunning, T. H.; Hay, P. J. Modern Theoretical Chemistry; Schaefer, H. F., Ed.; Plenum: New York, 1977.

(15) Hay, P. J.; Wadt, W. R. Ab Initio Effective Core Potentials for Molecular Calculations. Potentials for $\mathrm{K}$ to Au Including the Outermost Core Orbitals. The Journal of Chemical Physics 1985, 82 (1), 299-310. 
(16) Grimme, S. Semiempirical Hybrid Density Functional with Perturbative SecondOrder Correlation. The Journal of Chemical Physics 2006, 124 (3), 034108.

(17) É. Brémond and C. Adamo, "Seeking for parameter-free double-hybrid functionals: The PBE0DH model," The Journal of Chemical Physics, 2011, 135, 024106.

(18) Yanai, T.; Tew, D. P.; Handy, N. C. A New Hybrid Exchange-Correlation Functional Using the Coulomb-Attenuating Method (CAM-B3LYP). Chemical Physics Letters 2004, 393 (1-3), 51-57.

(19) Martin, R. L. Natural Transition Orbitals. The Journal of Chemical Physics 2003, 118 (11), 4775-4777. 